\title{
Criteria of irreducibility of the Koopman representations for the group $\mathrm{GL}_{0}(2 \infty, \mathbb{R})$
}

\author{
A.V. Kosyak \\ Max-Planck-Institut für Mathematik, Vivatsgasse 7, D-53111 Bonn, Germany \\ Institute of Mathematics, Ukrainian National Academy of Sciences, 3 Tereshchenkivs'ka \\ Str., Kyiv, 01601, Ukraine
}

\begin{abstract}
Our aim is to find the irreducibility criteria for the Koopman representation, when the group acts on some space with a measure (Conjecture 1.5). Some general necessary conditions of the irreducibility of this representation are established. In the particular case of the group $\mathrm{GL}_{0}(2 \infty, \mathbb{R})=$ $\lim _{n} \mathrm{GL}(2 n-1, \mathbb{R})$, the inductive limit of the general linear groups we prove that these conditions are also the necessary ones. The corresponding measure is infinite tensor products of one-dimensional arbitrary Gaussian noncentered measures. The corresponding $G$-space $X_{m}$ is a subspace of the space $\operatorname{Mat}(2 \infty, \mathbb{R})$ of infinite in both directions real matrices. In fact, $X_{m}$ is a collection of $m$ infinite in both directions rows. This result was announced in [20]. We give the proof only for $m \leq 2$. The general case will be studied later.
\end{abstract}

Keywords: infinite-dimensional groups, irreducible representation, Koopman's representation, Ismagilov's conjecture, Schur-Weyl duality, quasi-invariant, ergodic measure

2008 MSC: 22E65, (28C20, 43A80, 58D20)

Email address: kosyak02@gmail .com (A.V. Kosyak ) 


\section{Contents}

1 Introduction 2

1.1 Description of the dual for locally compact groups . . . . . . . 2

1.2 Regular, quasiregular and induced representations for infinite-

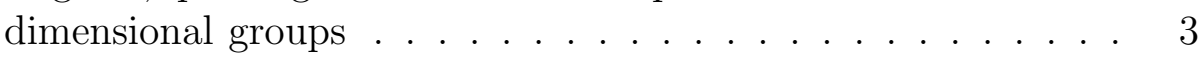

1.3 Koopman representation . . . . . . . . . . . . . . 4

1.4 Schur-Weyl duality . . . . . . . . . . . . . . . . . 5

1.5 The Dixmier commutation theorem, locally compact groups . 6

1.6 G-action and irreducibility of the Koopman representation . . 7

1.7 Counterexample to Conjecture 1.6 . . . . . . . . . . . . . . 8

1.7.1 Case $X=S_{n-1} \backslash S_{n} \ldots \ldots \ldots$. . . . . . . 8

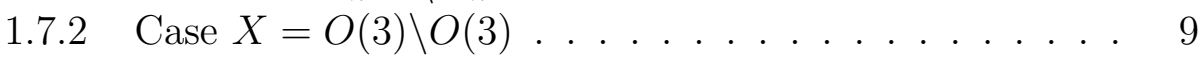

1.7.3 Centralizer of $S O(2 k+1) \ldots \ldots$

2 Representations of the inductive limit of the general linear groups $\mathrm{GL}_{0}(2 \infty, \mathbb{R}) \quad \mathbf{1 0}$

2.1 Finite-dimensional case . . . . . . . . . . . . . . . . . 10

2.2 Infinite-dimensional case . . . . . . . . . . . . . . 10

3 The proof of the irreducibility

3.1 The cases $m=1$. . . . . . . . . . . . . . . . . . 13

4 The proof of the irreducibility in the cases $m=2$

4.1 Some orthogonality problem in measure theory . . . . . . . . . 16

4.2 Approximation of $x_{k n}$ and $D_{k n} \ldots \ldots \ldots$. . . . . . 18

4.3 The proof of Lemmas $2.2,4.1$. . . . . . . . . . . . . . . . . . 33

4.4 The explicit expression for $\left(D^{-1}(\lambda) \mu, \mu\right) \ldots \ldots . . . . . .46$

4.5 The proof of Lemmas $4.2-4.7 \ldots \ldots$. . . . . . . . 48

\section{Introduction}

\subsection{Description of the dual for locally compact groups}

The main problem in the representation theory for a locally compact group $G$ is to find the set of all unitary irreducible representations of $G$ up to unitary equivalence and to decompose reducible representations into a direct sum or direct integral of irreducible. This set is called the unitary dual of $G$ and is denoted by $\hat{G}$. For many locally compact groups this problem has 
been solved, but for some particular cases it remains open, for example, for the group $\mathrm{SO}(p, q)$. To find the dual for locally compact groups $G$, one can use regular, quasiregular or induced representations. In the case of locally compact groups all these constructions are based on the existence of the invariant Haar measure on the initial group $G$ or some $G$-quasi-invariant measure on the corresponding homogeneous space $H \backslash G$, where $H$ is a closed subgroup of $G$ or on some general $G$-space $X$.

\subsection{Regular, quasiregular and induced representations for infinite-dimensional groups}

It is well known that there is no general method to describe $\hat{G}$ for infinitedimensional groups $G$. Our aim is to start the development of the harmonic analysis on infinite-dimensional groups.

In the previous articles we have generalized the notions of the regular, quasiregular and induced representations for infinite-dimensional groups by constructing $G$-quasi-invariant measures on suitable completions of the corresponding objects (groups, homogeneous spaces and $G$-spaces). In addition, we study the irreducibility of the constructed representations in the framework of the Ismagilov conjecture (see 1.1).

In this article we consider the case when the infinite-dimensional group $G$, the inductive limit of the general linear groups, acts on the space of $m$ infinite rows equipped with the Gaussian measure. We establish the criteria of irreducibility of constructed representations (see Theorem 2.1) in terms of the corresponding measure and express some general conjectures dealing with the irreducibility. These conjectures are natural generalization of the Ismagilov conjecture (see Conjecture 1.5).

Recall some previous constructions. Regular representations for infinitedimensional groups were defined and studied in [14, 15, 16]. Due to the result of A.Weil [30], there is no invariant measure on non locally compact groups. Therefore, to construct an analogue of a regular representation of an infinite-dimensional group $G$ we can, for example, construct a $G$-quasiinvariant measure on a suitable completion $\tilde{G}$ of the initial group $G$. The regular representation of an infinite-dimensional group can be irreducible, which never happens for a locally compact group, except for the trivial one!

To define a quasiregular representation we should construct a $G$-quasiinvariant measure on a suitable completion $\tilde{H} \backslash \tilde{G}$ of the homogeneous space $H \backslash G$ [17, 18, 19]. 
To construct the induced representation for infinite-dimensional groups we need to extend by continuity the representation of the subgroup $H$ to the corresponding completion $\tilde{H}$. The general construction of the induced representations and the beginning of the orbit methods for infinite-dimensional group of upper triangular matrices were done in [22].

To construct the regular representation for an infinite-dimensional group $G$, first we should find some larger topological group $\widetilde{G}$ and a measure $\mu$ on $\widetilde{G}$ such that $G$ is a dense subgroup in $\widetilde{G}$, and $\mu^{R_{t}} \sim \mu$ for all $t \in G$, (or $\mu^{L_{t}} \sim \mu$ for all $t \in G$ ), here $\sim$ means equivalence. The right and left representations $T^{R, \mu}, T^{L, \mu}: G \rightarrow U\left(L^{2}(\tilde{G}, \mu)\right)$ are naturally defined in the Hilbert space $L^{2}(\tilde{G}, \mu)$ by the following formulas:

$$
\begin{gathered}
\left(T_{t}^{R, \mu} f\right)(x)=(d \mu(x t) / d \mu(x))^{1 / 2} f(x t), \\
\left(T_{s}^{L, \mu} f\right)(x)=\left(d \mu\left(s^{-1} x\right) / d \mu(x)\right)^{1 / 2} f\left(s^{-1} x\right) .
\end{gathered}
$$

The right regular representation of infinite-dimensional groups can be irreducible if no left actions are admissible for the measure $\mu$, i.e., when $\mu^{L_{t}} \perp \mu$ for all $t \in G \backslash\{e\}$. In this case a von Neumann algebra $\mathfrak{A}^{T^{L, \mu}}$ generated by the left regular representation $T^{L, \mu}$ is trivial. More precisely:

Conjecture 1.1 (Ismagilov, 1985). The right regular representation

$$
T^{R, \mu}: G \rightarrow U\left(L^{2}(\tilde{G}, \mu)\right)
$$

is irreducible if and only if

1) $\mu^{L_{t}} \perp \mu \forall t \in G \backslash\{e\}$, (where $\perp$ stands for singular),

2) the measure $\mu$ is $G$-ergodic.

This conjecture was verified for a lot of particular cases. In the general case, it is an open problem. In the case of a finite field $\mathbb{F}_{p}$ we need some additional conditions for the irreducibility [21].

\subsection{Koopman representation}

Let $\alpha: G \rightarrow \operatorname{Aut}(X)$ be a measurable action of a group $G$ on a measurable space $(X, \mu)$ with $G$-quasi-invariant measure $\mu$, i.e, $\mu^{\alpha_{t}} \sim \mu$ for all $t \in G$. With these date one can associate the representation $\pi^{\alpha, \mu, X}: G \rightarrow$ $U\left(L^{2}(X, d \mu)\right)$, by the following formula:

$$
\left(\pi_{t}^{\alpha, \mu, X} f\right)(x)=\left(d \mu\left(\alpha_{t^{-1}}(x)\right) / d \mu(x)\right)^{1 / 2} f\left(\alpha_{t^{-1}}(x)\right), \quad f \in L^{2}(X, \mu) .
$$

In the case of an invariant measure this representation called Koopman's representation, see [13]. We would like to solve the following problems: 
Problem 1.2. Find criteria of irreducibility of the representation $\pi^{\alpha, \mu, X}$ defined by (1.1).

Problem 1.3. Find the description of the commutant of the von Neumann algebra generated by representation $\pi^{\alpha, \mu, X}$ when representation is reducible.

To study properties of the Koopman representation, in particular, the irreducibility, we need some conjectures to describe the commutant of the von Neumann algebras generated by this representation. The Schur-Weyl duality and the Dixmier commutation theorem below give us a very good hint for such a conjecture, see Conjecture 1.6 in a general context.

\subsection{Schur-Weyl duality}

Schur-Weyl duality [25, 26, 31] is a typical situation in representation theory involving two kinds of symmetry that determine each other.

From [32]: "If $V$ is a finite-dimensional complex vector space, then the symmetric group $S_{n}$ naturally acts on the tensor power $V^{\otimes n}$ by permuting the factors. This action of $S_{n}$ commutes with the action of $\operatorname{GL}(V)$, so all permutations $\sigma: V^{\otimes n} \rightarrow V^{\otimes n}$ are morphisms of GL( $\left.V\right)$-representations. This defines a morphism $\mathbb{C}\left[S_{n}\right] \rightarrow \operatorname{End}_{\mathrm{GL}(V)}\left(V^{\otimes n}\right)$, and a natural question to ask is whether this map is surjective.

Part of Schur-Weyl duality asserts that the answer is yes. The double commutant theorem plays an important role in the proof and also highlights an important corollary, namely that $V^{\otimes n}$ admits a canonical decomposition

$$
V^{\otimes n}=\bigoplus_{\lambda} V_{\lambda} \otimes S_{\lambda}
$$

where $\lambda$ runs over partitions, $V_{\lambda}$ are some irreducible representations of $\mathrm{GL}(V)$, and $S_{\lambda}$ are the Specht modules, which describe all irreducible representations of $S_{n}$. This gives a fundamental relationship between the representation theories of the general linear and symmetric groups; in particular, the assignment $V \mapsto V_{\lambda}$ can be upgraded to a functor called a Schur functor, generalizing the construction of the exterior and symmetric products."

Let $\operatorname{dim} V=m$ then $\mathrm{GL}(V)=\mathrm{GL}(m, \mathbb{C})$. The abstract form of the Schur-Weyl duality asserts that two algebras of operators on the tensor space generated by the actions of $\mathrm{GL}(m, \mathbb{C})$ and $S_{n}$ are the full mutual centralizers in the algebra of the endomorphisms $\operatorname{End}_{\mathbb{C}}\left(\mathbb{C}^{m} \otimes \mathbb{C}^{m} \otimes \cdots \otimes \mathbb{C}^{m}\right)$. 
Denote by $\alpha$ and $\beta$ the corresponding actions of $S_{n}$ and $\mathrm{GL}(m, \mathbb{C})$ in the group of all automorphisms Aut $\left(\mathbb{C}^{m} \otimes \mathbb{C}^{m} \otimes \cdots \otimes \mathbb{C}^{m}\right)$ :

$$
\alpha: S_{n} \rightarrow \operatorname{Aut}(X), \quad \beta: \operatorname{GL}(m, \mathbb{C}) \rightarrow \operatorname{Aut}(X) .
$$

Let $M^{\prime}$ be the commutant of the subset $M$ in the von Neumann algebra $B(H)$ of all bounded operators in a Hilbert space $H$ :

$$
M^{\prime}=\{B \in B(H) \mid[B, a]=0 \forall a \in M\} \text { where }[B, a]=B a-a B .
$$

Set $M_{1}=\left(\alpha\left(S_{n}\right)\right)^{\prime \prime}$ and $M_{2}=(\beta(\mathrm{GL}(m, \mathbb{C})))^{\prime \prime}$ then the Schur-Weyl duality states that $M_{1}^{\prime}=M_{2}$ hence, $M_{2}^{\prime}=M_{1}$.

In [27] the authors extend the classical Schur-Weyl duality between representations of the groups $\mathrm{SL}(m, \mathbb{C})$ and $S_{n}$ to the case of $\mathrm{SL}(m, \mathbb{C})$ and the infinite symmetric group $S_{\infty}$. In [24] the authors extend Weyl results to the classical infinite-dimensional locally finite algebras $\mathfrak{g} l_{\infty}, \mathfrak{s} l_{\infty}, \mathfrak{s} p_{\infty}, \mathfrak{s} o_{\infty}$.

\subsection{The Dixmier commutation theorem, locally compact groups}

Let $G$ be a locally compact group and let $h$ be the right invariant Haar measure on $G$, i.e., $h^{R_{t}}=h$ for all $t \in G$. Consider the left $L$ and the right $R$ action of the group $G$ on itself:

$$
R_{t}(x)=x t^{-1}, L_{s}(x)=s x, x, t, s \in G .
$$

The right and the left regular representations of the group $G$ are defined in the Hilbert space $L^{2}(G, h)$ by

$$
\left(\rho_{t} f\right)(x)=f(x t), \quad\left(\lambda_{s} f\right)(x)=\left(d h\left(s^{-1} x\right) / d h(x)\right)^{-1 / 2} f\left(s^{-1} x\right), f \in L^{2}(G, h),
$$

where $d h\left(s^{-1} x\right) / d h(x)$ is the Radon-Nikodim derivative.

Theorem 1.4 (Dixmier's commutation theorem [5]). The commutant of the von-Neumann algebra generated by the right regular representation is generated by the left regular representation. More precisely, let $\rho, \lambda: G \rightarrow$ $U\left(L^{2}(G, h)\right)$ be the right and the left regular representations of the group $G$, and let $\mathfrak{A}^{\rho}=\left(\rho_{t} \mid t \in G\right)^{\prime \prime}$ and $\mathfrak{A}^{\lambda}=\left(\lambda_{s} \mid s \in G\right)^{\prime \prime}$ be the corresponding von Neumann algebras. Then

$$
\left(\mathfrak{A}^{\rho}\right)^{\prime}=\mathfrak{A}^{\lambda} \quad \text { and } \quad\left(\mathfrak{A}^{\lambda}\right)^{\prime}=\mathfrak{A}^{\rho} .
$$




\subsection{G-action and irreducibility of the Koopman representation}

In both examples we have two commuting actions of the group $G_{1}$ and $G_{2}$ on the same space $X$. Let $Z_{G}(H)$ be a centralizer of the subgroup $H$ in the group $G$ :

$$
Z_{G}(H)=\{g \in G \mid\{g, a\}=e \forall a \in H\},
$$

where $\{g, a\}=g a g^{-1} a^{-1}$. In the first example, we have two commuting actions $\alpha$ and $\beta$ of the groups $G_{1}=S_{n}$ and $G_{2}=\operatorname{GL}(n, \mathbb{C})$ on the space $X$ such that $Z_{\text {Aut }(X)}\left(\alpha\left(G_{1}\right)\right) \supseteq \beta\left(G_{2}\right)$. In the second example, we have two commuting actions $R$ and $L$ of the same group $G$ in the space $X=G$. In this case we have $\{R(G), L(G)\}=e$ or $Z_{\operatorname{Aut}(G)}(R(G)) \supseteq L(G)$. In the general case, if we have only one group $G$ acting via $\alpha$ on the space $X$, the second group should be the centralizer of the group $\alpha(G)$ in the group $\operatorname{Aut}(X)$, i.e., it is natural to consider $G_{2}=Z_{\operatorname{Aut}(X)}(\alpha(G))$.

Come back to the Koopman representation (1.1). Consider the centralizer $Z_{\text {Aut }(X)}(\alpha(G))$ of the subgroup $\alpha(G)=\left\{\alpha_{t} \mid t \in G\right\}$ in the group $\operatorname{Aut}(X)$ and its subgroup $G_{2}$ defined as follows:

$$
G_{2}:=Z_{\operatorname{Aut}(X)}^{\mu}(\alpha(G)):=\left\{g \in Z_{\operatorname{Aut}(X)}(\alpha(G)) \mid \mu^{g} \sim \mu\right\} .
$$

Define the representation $T$ of the group $G_{2}$ as follows:

$$
\left(T_{g} f\right)(x)=(d \mu(g x) / d \mu(x))^{1 / 2} f(g x) .
$$

Consider two von Neumann algebras

$$
\mathfrak{A}^{\pi}(G)=\left(\pi_{t} \mid t \in G\right)^{\prime \prime}, \quad \mathfrak{A}^{T}\left(G_{2}\right)=\left(T_{g} \mid g \in G_{2}\right)^{\prime \prime} .
$$

The conditions 1) and 2) below are necessary conditions of the irreducibility

of the representation $\pi^{\alpha, \mu, X}$. It would be interesting to know when they are sufficient, i.e., when the following conjecture is true

Conjecture 1.5 (Kosyak, [16, 18]). The representation

$$
\pi^{\alpha, \mu, X}: G \rightarrow U\left(L^{2}(X, \mu)\right)
$$

is irreducible if and only if

1) $\mu^{g} \perp \mu \forall g \in Z_{\operatorname{Aut}(X)}(\alpha(G)) \backslash\{e\}$,

2) the measure $\mu$ is $G$-ergodic. 
Recall that a measure $\mu$ is $G$-ergodic if $f\left(\alpha_{t}(x)\right)=f(x) \mu$ a.e. for all $t \in G$ implies $f(x)=$ const $\mu$ a.e.(almost everywhere) for all functions $f \in L^{1}(X, \mu)$.

Conjecture 1.6. The commutant of the von Neumann algebra generated by representation $\pi$ (1.1) of the group $G$ coincides with the von Neumann algebra generated by the representation $T$ (1.4) of the subgroup $G_{2}$ in the centralizer $Z_{\mathrm{Aut}(X)}(\alpha(G))$ :

$$
\left(\mathfrak{A}^{\pi}(G)\right)^{\prime}=\mathfrak{A}^{T}\left(G_{2}\right) .
$$

For a lot of particular cases Conjecture 1.6 holds, but in general it fails. Below we give several example for which Conjecture 1.6 fails.

\subsection{Counterexample to Conjecture 1.6}

1.7.1. Case $X=S_{n-1} \backslash S_{n}$

Example 1.1. Consider the group $S_{n}$ acting on the homogeneous space $X=$ $S_{n-1} \backslash S_{n}$. For corresponding right quasiregular representation of $S_{n}$ in $L^{2}(X)$ Conjecture 1.6 fails.

Proof. To simplify details set $n=3$. For general $n$ the proof is the same. Let $\sigma_{1}, \sigma_{2}$ be two generators of the group $S_{3}$ :

$$
S_{3}=\left(\sigma_{1}, \sigma_{2} \mid \sigma_{1}^{2}=e, \sigma_{2}^{2}=e, \sigma_{1} \sigma_{2} \sigma_{1}=\sigma_{2} \sigma_{1} \sigma_{2}\right)
$$

Let the group $S_{2}$ is generated by $\sigma_{1}$, then the space $X$ consists of three classes $x_{0}=\left\{e, \sigma_{1}\right\}, x_{1}=\left\{\sigma_{2}, \sigma_{1} \sigma_{2}\right\}, x_{3}=\left\{\sigma_{2} \sigma_{1}, \sigma_{1} \sigma_{2} \sigma_{1}\right\}$. The right action of $S_{3}$ on the space $X$ is as follows:

$$
\begin{aligned}
& x_{0} \sigma_{1}=x_{0}, \quad x_{1} \sigma_{1}=x_{2}, \quad x_{2} \sigma_{1}=x_{1}, \\
& x_{0} \sigma_{2}=x_{1}, \quad x_{1} \sigma_{2}=x_{0}, \quad x_{2} \sigma_{2}=x_{2} .
\end{aligned}
$$

Therefore, in $L^{2}(X)$ the corresponding representations for $T_{\sigma_{1}}$ and $T_{\sigma_{2}}$ are as follows:

$$
T_{\sigma_{1}}=\left(\begin{array}{lll}
1 & 0 & 0 \\
0 & 0 & 1 \\
0 & 1 & 0
\end{array}\right), \quad T_{\sigma_{2}}=\left(\begin{array}{lll}
0 & 1 & 0 \\
1 & 0 & 0 \\
0 & 0 & 1
\end{array}\right) \text {. }
$$

The representation $T$ is reducible, since the vector $e_{0}+e_{1}+e_{2}$ is invariant. It splits into one-dimensional and two- dimensional irreducible representations. But the group $S_{3}$ acts on $X$ by permutations so, its centralizer is trivial. 


\subsubsection{Case $X=O(3) \backslash O(3)$}

Example 1.2. Consider the group $O(3)$ acting on the homegeneous space $O(3) \backslash O(3) \simeq S^{2}$. The centralizer of $O(3)$ in the group of all automorphisms Aut $\left(S^{2}\right)$ consists of two elements $I$ and $-I$ by Lemma 1.7 but the representation of $O(3)$ in $L^{2}(X)$ is an infinite direct sum of irreducible representations generated by eigenvectors of the Laplace operator on $S^{2}$, see [8, Chapter I,§3]. Therefore, Conjecture 1.6 fails.

\subsubsection{Centralizer of $S O(2 k+1)$}

Let $n \geq 0$, and $S O(n)$ be the group of all real orthogonal $n \times n$-matrices with determinant 1 . This group effectively and transitively acts $n-1$ dimensional sphere $S^{n-1}$, and so it can be regarded as a subgroup of the group $\mathcal{H}\left(S^{n-1}\right)$ of all homeomorphisms of $S^{n-1}$.

Let $I$ be the unit matrix. Then $-I$ is an "antipodal" map, that is $-I(p)=$ $-p$ for all $p \in S^{n-1}$. Evidently, $I$ and $-I$ commute with all elements from $S O(n)$, and so $\{ \pm I\}$ belongs to the centralizer of $S O(n)$ in $O(n)$.

Lemma 1.7. Suppose $n=2 k+1$ is odd. Then the group $\{ \pm I\}$ is the centralizer of $S O(2 k+1)$ in all the group $\mathcal{H}\left(S^{2 k}\right)$.

Proof. (given by S. Maximenko.) Suppose $h \in \mathcal{H}\left(S^{n-1}\right)$ commutes with all matrices $A \in S O(n)$, that is $h \circ A(x)=A \circ h(x)$ for all $x \in S^{n-1}$. We should prove that then $h= \pm I$.

First we claim that $h(x) \in\{ \pm x\}$ for each $x \in S^{n-1}$. Indeed, since $n$ is odd, for each $x \in S^{n-1}$ there exists $A \in S O(n)$ such that $\{ \pm x\}$ is the set of all fixed points for $A$. Hence

$$
h(x)=h \circ A(x)=A \circ h(x),
$$

that is $h(x)$ is a fixed point for $A$, and so $h(x)= \pm x$.

Now, suppose $h(x)=\varepsilon x$ for some $\varepsilon= \pm 1$. We claim that then $h=\varepsilon I$. Let $F=\left\{x \in S^{n-1} \mid h(x)=\varepsilon x\right\}$ be the set of points where $h$ coincides with $\varepsilon I$. We will show that $F$ is a non-empty open-closed subset of $S^{n-1}$, which will imply that $F$ coincides with all of $S^{n-1}$.

As shown above $x \in F$, so $F \neq \varnothing$. Moreover, as $h$ and $-I$ are continuous, $F$ is closed. It remains to show that $F$ is open. Let $U$ be a small neighbourhood of $x$ such that $U \cap-U=\varnothing$, that is $U$ does not contain antipodal pairs. Since $h$ is continuous and $h(x)=\varepsilon x \in \varepsilon U$, there exists a neighbourhood $V$ of $x$ such that $h(V)=\varepsilon U$. Then for each $y \in V$ we have 
that $h(y) \in\{ \pm y\} \cap \varepsilon U=\varepsilon y$. In other words, $h=\varepsilon I$ on $V$, and so $V \subset F$. This proves that $F=S^{n-1}$.

\section{Representations of the inductive limit of the general linear groups $\mathrm{GL}_{0}(2 \infty, \mathbb{R})$}

\subsection{Finite-dimensional case}

Consider the space $X_{m, n}=\left\{x=\sum_{1 \leq k \leq m} \sum_{-n \leq r \leq n} x_{k r} E_{k r}, x_{k r} \in \mathbb{R}\right\}$, with the measure (see (2.4) $) \mu_{(b, a)}^{m, n}(x)=\otimes_{k=1}^{m} \otimes_{-n \leq r \leq n} \mu_{\left(b_{k r}, a_{k r}\right)}\left(x_{k r}\right)$. On the space $X_{m, n}$ acts two groups $\operatorname{GL}(m, \mathbb{R})$ from the left and $\mathrm{GL}(2 n+1, \mathbb{R})$ from the right and these actions commute. Therefore, two von Neumann algebras $\mathfrak{A}_{1}$ and $\mathfrak{A}_{2, n}$ in the Hilbert space $L^{2}\left(X_{m, n}, \mu_{(b, a)}^{m, n}\right)$ generated respectively by the left and the right actions of the corresponding groups have the property that $\mathfrak{A}_{1}^{\prime} \subseteq \mathfrak{A}_{2, n}$. We study what happens when $n \rightarrow \infty$. As the limit we obtain some unitary representation of the group $\mathrm{GL}_{0}(2 \infty, \mathbb{R})=\lim _{n, i^{s}} \mathrm{GL}(2 n-1, \mathbb{R})$ (see below). In generic case, this representation is reducible, namely, if there exists a non trivial element $s \in \mathrm{GL}(m, \mathbb{R})$ such the the left action is admissible for the measure $\mu_{(b, a)}^{m}$, i.e., $\left(\mu_{(b, a)}^{m}\right)^{L_{s}} \sim \mu_{(b, a)}^{m}$. But when no non-trivial left actions are admissible, i.e., when $\left(\mu_{(b, a)}^{m}\right)^{L_{s}} \perp \mu_{(b, a)}^{m}$ for all $s \in \mathrm{GL}(m, \mathbb{R}) \backslash\{e\}$ we prove that this representation is irreducible Theorem 2.1. Here, as in the case of the regular [14, 15] and quasiregular [17, 18] representations of the group $B_{0}^{\mathbb{N}}$ we obtain the remarkable fact that the irreducible representations can be obtained as the inductive limit of reducible representations!

\subsection{Infinite-dimensional case}

Let us denote by $\operatorname{Mat}(2 \infty, \mathbb{R})$ the space of all real matrices infinite in both directions:

$$
\operatorname{Mat}(2 \infty, \mathbb{R})=\left\{x=\sum_{k, n \in \mathbb{Z}} x_{k n} E_{k n}, x_{k n} \in \mathbb{R}\right\},
$$

where $E_{k n}, k, n \in \mathbb{Z}$ are infinite matrix unities.

The group $\mathrm{GL}_{0}(2 \infty, \mathbb{R})=\lim _{n, i^{s}} \mathrm{GL}(2 n-1, \mathbb{R})$ is defined as the inductive limit of the general linear groups $G_{n}=\operatorname{GL}(2 n-1, \mathbb{R})$ with respect to the symmetric embedding $i^{s}(2.2)$ :

$\mathrm{GL}(2 n-1, \mathbb{R}) \ni x \mapsto i_{n+1}^{s}(x)=x+E_{-(n+1),-(n+1)}+E_{n+1, n+1} \in \mathrm{GL}(2 n+1, \mathbb{R})$. 
We consider a $G$-space $X_{m}, m \in \mathbb{N}$ as the following subspace of the space $\operatorname{Mat}(2 \infty, \mathbb{R})$ :

$$
X_{m}=\left\{x \in \operatorname{Mat}(2 \infty, \mathbb{R}) \mid x=\sum_{k=1}^{m} \sum_{n \in \mathbb{Z}} x_{k n} E_{k n}\right\} .
$$

The group $\mathrm{GL}_{0}(2 \infty, \mathbb{R})$ acts from the right on the space $X_{m}$. Namely, the right action of the group $G=\mathrm{GL}_{0}(2 \infty, \mathbb{R})$ is correctly defined on the space $X_{m}$ by the formula $R_{t}(x)=x t^{-1}, t \in G, x \in X_{m}$. We define a Gaussian noncentered product measure $\mu^{m}=\mu_{(b, a)}^{m}$ on the space $X_{m}$ :

$$
\mu_{(b, a)}^{m}(x)=\otimes_{k=1}^{m} \otimes_{n \in \mathbb{Z}} \mu_{\left(b_{k n}, a_{k n}\right)}\left(x_{k n}\right)
$$

where

$$
d \mu_{\left(b_{k n}, a_{k n}\right)}\left(x_{k n}\right)=\left(b_{k n} / \pi\right)^{1 / 2} \exp \left(-b_{k n}\left(x_{k n}-a_{k n}\right)^{2}\right) d x_{k n}
$$

and $b=\left(b_{k n}\right)_{k, n}, b_{k n}>0, a=\left(a_{k n}\right)_{k, n}, a_{k n} \in \mathbb{R}, 1 \leq k \leq m, n \in \mathbb{Z}$. Define the representation $T^{R, \mu, m}$ of the group $\mathrm{GL}_{0}(2 \infty, \mathbb{R})$ in the space $L^{2}\left(X_{m}, \mu_{(b, a)}^{m}\right)$ by the formula:

$$
\left(T_{t}^{R, \mu, m} f\right)(x)=\left(d \mu_{(b, a)}^{m}(x t) / d \mu_{(b, a)}^{m}(x)\right)^{1 / 2} f(x t), f \in L^{2}\left(X_{m}, \mu_{(b, a)}^{m}\right) .
$$

Obviously, the centralizer $Z_{\operatorname{Aut}\left(X_{m}\right)}(\alpha(G)) \subset \operatorname{Aut}\left(X_{m}\right)$ contains the group $L(\mathrm{GL}(m, \mathbb{R}))$, i.e., the image of the group $\operatorname{GL}(m, \mathbb{R})$ with respect to the left action $L: \operatorname{GL}(m, \mathbb{R}) \rightarrow \operatorname{Aut}\left(X_{m}\right), L_{s}(x)=s x, s \in \mathrm{GL}(m, \mathbb{R}), x \in X_{m}$. We prove the following theorem for $m \leq 2$.

Theorem 2.1. The representation $T^{R, \mu, m}: \mathrm{GL}_{0}(2 \infty, \mathbb{R}) \rightarrow U\left(L^{2}\left(X_{m}, \mu_{(b, a)}^{m}\right)\right)$ is irreducible if and only if $\left(\mu_{(b, a)}^{m}\right)^{L_{s}} \perp \mu_{(b, a)}^{m} \forall s \in \mathrm{GL}(m, \mathbb{R}) \backslash\{e\}$.

Remark 2.1. Any Gaussian product-measure $\mu_{(b, a)}^{m}$ on $X_{m}$ is $\mathrm{GL}_{0}(2 \infty, \mathbb{R})$ right-ergodic [28, $\S 3$, Corollary 1]. For non-product-measures this is not true in general.

To study the condition $\left(\mu_{(b, a)}^{m}\right)^{L_{t}} \perp \mu_{(b, a)}^{m}$ for $t \in \mathrm{GL}(m, \mathbb{R})$ set

$t=\left(t_{r s}\right)_{r, s=1}^{m} \in \mathrm{GL}(m, \mathbb{R}), \quad B_{n}=\operatorname{diag}\left(b_{1 n}, b_{2 n}, \ldots, b_{m n}\right), \quad X_{n}(t)=B_{n}^{1 / 2} t B_{n}^{-1 / 2}$.

Let $M_{j_{1} j_{2} \ldots j_{r}}^{i_{1} i_{2} \ldots i_{r}}(t)$ be the minors of the matrix $t$ with $i_{1}, i_{2}, \ldots, i_{r}$ rows and $j_{1}, j_{2}, \ldots, j_{r}$ columns, $1 \leq r \leq m$. Let $\delta_{r s}$ be the Kronecker symbols. 
Lemma 2.2. For the measures $\mu_{(b, a)}^{m}, m \in \mathbb{N}$ the relation $\left(\mu_{(b, a)}^{m}\right)^{L_{t}} \perp \mu_{(b, a)}^{m}$ $\forall t \in \mathrm{GL}(m, \mathbb{R}) \backslash\{e\}$ holds if and only if

$$
\begin{gathered}
\prod_{n \in \mathbb{Z}} \frac{1}{2^{m}|\operatorname{det} t|} \operatorname{det}\left(I+X_{n}^{*}(t) X_{n}(t)\right)+\sum_{n \in \mathbb{Z}} \sum_{r=1}^{m} b_{r n}\left(\sum_{s=1}^{m}\left(t_{r s}-\delta_{r s}\right) a_{s n}\right)^{2}=\infty, \\
1+\sum_{r=1}^{m} \sum_{\substack{1 \leq i_{1}<i_{2}<\ldots<i_{r} \leq m ; 1 \leq j_{1}<j_{2}<\ldots<j_{r} \leq m \\
\text { where }}}^{\operatorname{det}\left(I+X_{n}^{*}(t) X_{n}(t)\right)=}\left(M_{j_{1} j_{2} \ldots j_{r}}^{i_{1} i_{2} \ldots i_{r}}\left(X_{n}(t)\right)\right)^{2} .
\end{gathered}
$$

This lemma will be proved in Section 4.3.

Remark 2.2. The idea of the proof of the irreducibility. Let us denote by $\mathfrak{A}^{m}$ the von Neumann algebra generated by the representation $T^{R, \mu, m}$ : $\mathfrak{A}^{m}=\left(T_{t}^{R, \mu, m} \mid t \in G\right)^{\prime \prime}$. For $\alpha=\left(\alpha_{k}\right) \in\{0,1\}^{m}$ define the von Neumann algebra $L_{\alpha}^{\infty}\left(X_{m}, \mu^{m}\right)$ as follows:

$$
L_{\alpha}^{\infty}\left(X_{m}, \mu^{m}\right)=\left(\exp \left(i t B_{k n}^{\alpha}\right) \mid 1 \leq k \leq m, t \in \mathbb{R}, n \in \mathbb{Z}\right)^{\prime \prime},
$$

where $B_{k n}^{\alpha}=\left\{\begin{array}{lll}x_{k n}, & \text { if } & \alpha_{k}=0 \\ D_{k n}, & \text { if } & \alpha_{k}=1\end{array}\right.$.

The proof of the irreducibility is based on three facts:

1) using the orthogonality condition $\left(\mu^{m}\right)^{L_{t}} \perp \mu^{m}$ for all $t \in \mathrm{GL}(m, \mathbb{R}) \backslash\{e\}$ we can approximate by generators $A_{k n}=A_{k n}^{R, m}=\left.\frac{d}{d t} T_{I+t E_{k n}}^{R, \mu, m}\right|_{t=0}$ the set of operators $\left(B_{k n}^{\alpha}\right)_{k=1}^{m}, n \in \mathbb{Z}$ for some $\alpha \in\{0,1\}^{m}$ depending on the measure $\mu^{m}$,

2) the subalgebra $L_{\alpha}^{\infty}\left(X_{m}, \mu^{m}\right) \subset \mathfrak{A}^{m}$ is a maximal abelian subalgebra in $\mathfrak{A}^{m}$,

3) the measure $\mu^{m}$ is $G$-ergodic.

Here the generators $A_{k n}^{R, m}$ are given by the formulas:

$$
A_{k n}^{R, m}=\sum_{r=1}^{m} x_{r k} D_{r n}, \quad k, n \in \mathbb{Z}, \quad \text { where } \quad D_{k n}=\partial / \partial x_{k n}-b_{k n}\left(x_{k n}-a_{k n}\right) .
$$

Remark 2.3. The fact that conditions $\left(\mu^{m}\right)^{L_{t}} \perp \mu^{m}$ for all $t \in \mathrm{GL}(m, \mathbb{R}) \backslash\{e\}$ implies the possibility of the approximation of $x_{k n}$ and $D_{k n}$ is based on some completely independent statement about the properties of projections of two infinite vectors $f=\left(f_{k}\right)_{k \in \mathbb{N}}$ and $g=\left(g_{k}\right)_{k \in \mathbb{N}}$ such that $f, g, f+s g \notin l_{2}$ for all $s \in \mathbb{R}$ (Lemma 4.10). This lemma is a key part of the proof of the irreducibility of the representation. 
Remark 2.4. Similarly, for the "nilpotent group" $B_{0}^{\mathbb{N}}$ and the infinite product of arbitrary Gaussian measures on $\mathbb{R}^{m}$ (see [2]) the proof of the irreducibility is based on another completely independent statement namely, Hadamard - Fischer's inequality, see Lemma 2.3.

Lemma 2.3 (Hadamard - Fischer's inequality [9], [10] ). For any positive definite matrix $C \in \operatorname{Mat}(m, \mathbb{R}), m \in \mathbb{N}$ and any two subsets $\alpha$ and $\beta$ with $\emptyset \subseteq \alpha, \beta \subseteq\{1, \ldots, m\}$ the following inequality holds:

$$
\left|\begin{array}{cc}
M(\alpha) & M(\alpha \bigcap \beta) \\
M(\alpha \bigcup \beta) & M(\beta)
\end{array}\right|=\left|\begin{array}{cc}
A(\hat{\alpha}) & A(\hat{\alpha} \bigcup \hat{\beta}) \\
A(\hat{\alpha} \bigcap \hat{\beta}) & A(\hat{\beta})
\end{array}\right| \geq 0
$$

where $M(\alpha)=M_{\alpha}^{\alpha}(C), A(\alpha)=A_{\alpha}^{\alpha}(C)$ and $\hat{\alpha}=\{1, \ldots, m\} \backslash \alpha$.

For details see [9, p.573], [10, Chapter 2.5, problem 36].

The conditions of orthogonality $\mu^{L_{t}} \perp \mu$ with respect to the left action of the group $B(m, \mathbb{R})$ on $X^{m}$ were expressed as the divergence of some series, $S_{k n}^{L}(\mu)=\infty, 1 \leq k<n \leq m$. Conditions of the approximation of the variables $x_{k n}$ by combinations of generators $A_{p q}$ were expressed in terms of the divergence of another series $\Sigma_{k n}$. The proof of the fact that conditions $S_{k n}^{L}(\mu)=\infty, 1 \leq k<n \leq m$ imply conditions $\Sigma_{k n}=\infty, 1 \leq k<n \leq m$ is based on the Hadamard - Fischer's inequality.

\section{The proof of the irreducibility}

\subsection{The cases $m=1$}

As before, let us denote by $\left\langle f_{n} \mid n \in \mathbb{N}\right\rangle$ the closure of the linear space generated by the set of vectors $\left(f_{n}\right)_{n \in \mathbb{N}}$ in a Hilbert space $H$. We shall write $\mu_{(b, a)}=\mu_{(b, a)}^{1}$.

In the case $m=1$ the generators $A_{k n}^{R, 1}$ have the form

$$
A_{k n}^{R, 1}=x_{1 k} D_{1 n}, \quad k, n \in \mathbb{Z} .
$$

The following lemmas are proved in [1]

Lemma 3.1. The following three conditions are equivalent:

(i) $\left(\mu_{(b, a)}\right)^{L_{t}} \perp \mu_{(b, a)}$ for all $t \in G L(1, \mathbb{R}) \backslash\{e\}$,

(ii) $\left(\mu_{(b, a)}\right)^{L_{-E_{11}}} \perp \mu_{(b, a)}$,

(iii) $S_{11}^{L}(\mu)=4 \sum_{n \in \mathbb{Z}} b_{1 n} a_{1 n}^{2}=\infty$. 
Lemma 3.2. For $k, m \in \mathbb{Z}$ we have

$$
x_{1 k} x_{1 m} \mathbf{1} \in\left\langle A_{k n}^{R, 1} A_{m n}^{R, 1} \mathbf{1}=x_{1 k} x_{1 m} D_{1 n}^{2} \mathbf{1} \mid n \in \mathbb{Z}\right\rangle .
$$

Lemma 3.3. For any $k \in \mathbb{Z}$ we have

$$
x_{1 k} \mathbf{1} \in\left\langle x_{1 k} x_{1 n} \mathbf{1} \mid n \in \mathbb{Z}\right\rangle \Leftrightarrow S_{11}^{L}(\mu)=\infty .
$$

So, operators $x_{1 k}, k \in \mathbb{Z}$ are affiliated (see [6]) with the von Neumann algebra $\mathfrak{A}^{1}$ (notation $x_{1 k} \eta \mathfrak{A}^{1}$ ) which completes the proof of the irreducibility for $m=1$.

\section{The proof of the irreducibility in the cases $m=2$}

In the case $m=2$ the generators $A_{k n}:=A_{k n}^{R, 2}:=\left.\frac{d}{d t} T_{I+t E_{k n}}^{R, \mu, 2}\right|_{t=0}$ have the form:

$$
A_{k n}=x_{1 k} D_{1 n}+x_{2 k} D_{2 n}, \quad k, n \in \mathbb{Z}
$$

Lemma 4.1. Three following conditions (i)-(iii) are equivalent for the measure $\mu=\mu_{(b, a)}^{2}$ :

(i) $\mu^{L_{t}} \perp \mu$ for all $t \in G L(2, \mathbb{R}) \backslash\{e\}$, where $L_{t}(x)=t x, x \in X_{2}$;

$$
\begin{aligned}
& (i i)\left\{\begin{array}{lll}
(a) & \mu^{L_{\exp \left(t E_{12}\right)}} \perp \mu, & \forall t \in \mathbb{R} \backslash\{0\} \\
(b) & \mu^{L_{\exp \left(t E_{21}\right)} \perp \mu,}, \forall t \in \mathbb{R} \backslash\{0\} \\
(c) & \mu^{L_{\exp \left(t E_{12}\right) P_{1}} \perp \mu,}, \forall t \in \mathbb{R} \\
(d) & \mu^{L_{\exp \left(t E_{21}\right) P_{2}} \perp \mu,}, \forall t \in \mathbb{R} \\
(e) & \mu^{L_{\tau_{-}(\phi, s)}} \perp \mu, & \forall \tau_{-}(\phi, s) \in G L(2, \mathbb{R}) \backslash\{e\}
\end{array}\right.
\end{aligned}
$$

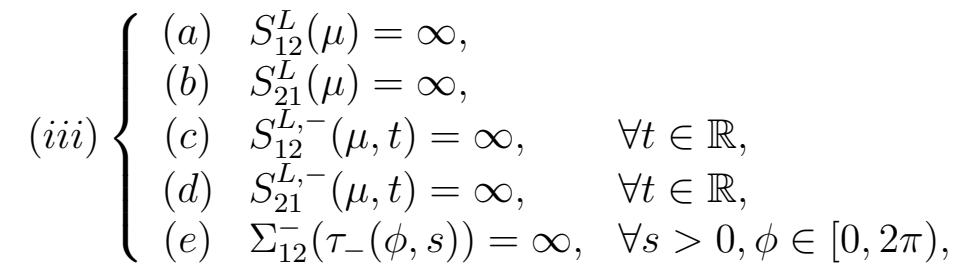

where

$$
S_{k n}^{L}(\mu)=\sum_{m \in \mathbb{Z}} \frac{b_{k m}}{2}\left(\frac{1}{2 b_{n m}}+a_{n m}^{2}\right), \quad k \neq n
$$




$$
\begin{aligned}
& S_{k n}^{L,-}(\mu, t)=\frac{t^{2}}{4} \sum_{m \in \mathbb{Z}} \frac{b_{k m}}{b_{n m}}+\sum_{m \in \mathbb{Z}} \frac{b_{k m}}{2}\left(-2 a_{k m}+t a_{n m}\right)^{2}, \\
& \Sigma_{12}^{-}\left(\tau_{-}(\phi, s)\right)=\sin ^{2} \phi \Sigma_{1}(s)+\Sigma_{2}^{-}\left(\tau_{-}(\phi, s)\right), \\
& \Sigma_{1}(s):=\sum_{n \in \mathbb{Z}}\left(s^{2} \sqrt{\frac{b_{1 n}}{b_{2 n}}}-s^{-2} \sqrt{\frac{b_{2 n}}{b_{1 n}}}\right)^{2}, \\
& \Sigma_{2}^{-}\left(\tau_{-}(\phi, s)\right):=\sum_{n \in \mathbb{Z}}\left(4 \sin ^{2} \frac{\phi}{2} b_{1 n}+4 \cos ^{2} \frac{\phi}{2} s^{-4} b_{2 n}\right)\left(\sin \frac{\phi}{2} a_{1 n}-s^{2} \cos \frac{\phi}{2} a_{2 n}\right)^{2}, \\
& \exp \left(t E_{12}\right)=I+t E_{12}=\left(\begin{array}{cc}
1 & t \\
0 & 1
\end{array}\right), \quad \exp \left(t E_{21}\right)=I+t E_{21}=\left(\begin{array}{ll}
1 & 0 \\
t & 1
\end{array}\right), \\
& \exp \left(t E_{12}\right) P_{1}=\left(\begin{array}{cc}
-1 & t \\
0 & 1
\end{array}\right), \quad \exp \left(t E_{21}\right) P_{2}=\left(\begin{array}{cc}
1 & 0 \\
t & -1
\end{array}\right) \\
& \tau_{-}(\phi, s)=\left(\begin{array}{cc}
\cos \phi & s^{2} \sin \phi \\
s^{-2} \sin \phi & -\cos \phi
\end{array}\right) \text { and } P_{1}=\left(\begin{array}{cc}
-1 & 0 \\
0 & 1
\end{array}\right), P_{2}=\left(\begin{array}{cc}
1 & 0 \\
0 & -1
\end{array}\right) \text {. }
\end{aligned}
$$

Moreover, $(i i)(\sharp) \Leftrightarrow(i i i)(\sharp)$ for $\sharp=a, b, c, d, e$.

Remark 4.1. We observe that

$\tau_{-}(\phi, s)=\left(\begin{array}{cc}\cos \phi & s^{2} \sin \phi \\ s^{-2} \sin \phi & -\cos \phi\end{array}\right)=\left(\begin{array}{cc}s & 0 \\ 0 & s^{-1}\end{array}\right)\left(\begin{array}{cc}\cos \phi & -\sin \phi \\ \sin \phi & \cos \phi\end{array}\right)\left(\begin{array}{cc}s^{-1} & 0 \\ 0 & s\end{array}\right) P_{2}$.

Remark 4.2. We note [12, Chapter V ,§8 Problems, 2, p. 147] that every element of $\mathrm{SL}(2, \mathbb{R})$ is conjugate to at least one matrix of the form

$$
\left(\begin{array}{cc}
a & 0 \\
0 & a^{-1}
\end{array}\right), a \neq 0,\left(\begin{array}{ll}
1 & t \\
0 & 1
\end{array}\right),\left(\begin{array}{cc}
-1 & t \\
0 & -1
\end{array}\right),\left(\begin{array}{cc}
\cos \phi & \sin \phi \\
-\sin \phi & \cos \phi
\end{array}\right) .
$$

Remark 4.3. The three following conditions are equivalent:

(ii) $\Sigma_{12}^{-}\left(\tau_{-}(\phi, s)\right)=\sin ^{2} \phi \Sigma_{1}(s)+\Sigma_{2}^{-}\left(\tau_{-}(\phi, s)\right)=\infty, \quad \phi \in[0,2 \pi), s>0$,

$$
\Sigma_{1}(s)+\Sigma_{2}\left(C_{1}, C_{2}\right)=\infty, \quad s>0,\left(C_{1}, C_{2}\right) \in \mathbb{R}^{2} \backslash\{0\},
$$

where $\Sigma_{1}(s)$ is defined by (4.3) and

$$
\Sigma_{2}\left(C_{1}, C_{2}\right):=\sum_{n \in \mathbb{Z}}\left(C_{1}^{2} b_{1 n}+C_{2}^{2} b_{2 n}\right)\left(C_{1} a_{1 n}+C_{2} a_{2 n}\right)^{2} .
$$


Proof. In Section 4.3 we shall show that $(i) \Leftrightarrow(i i)$ (see (4.66)), i.e., that

$$
\mu^{L_{\tau_{-}(\phi, s)}} \perp \mu \Leftrightarrow \Sigma_{12}^{-}\left(\tau_{-}(\phi, s)\right)=\sin ^{2} \phi \Sigma_{1}(s)+\Sigma_{2}^{-}\left(\tau_{-}(\phi, s)\right)=\infty .
$$

To prove $(i i) \Leftrightarrow($ iii) set

$$
\sin \frac{\psi}{2}=\sin \frac{\phi}{2}\left(\sin ^{2} \frac{\phi}{2}+s^{4} \cos ^{2} \frac{\phi}{2}\right)^{-1 / 2}, \quad \cos \frac{\psi}{2}=s^{2} \cos \frac{\phi}{2}\left(\sin ^{2} \frac{\phi}{2}+s^{4} \cos ^{2} \frac{\phi}{2}\right)^{-1 / 2}
$$

then using (4.4) we get

$$
\begin{gathered}
\Sigma_{2}^{-}\left(\tau_{-}(\phi, s)\right):=\left(\sin ^{2} \frac{\psi}{2}+s^{4} \cos ^{2} \frac{\psi}{2}\right)^{2} 4 \sum_{n \in \mathbb{Z}}\left(\sin ^{2} \frac{\psi}{2} b_{1 n}+s^{-8} \cos ^{2} \frac{\psi}{2} b_{2 n}\right) \times \\
\left(\sin \frac{\psi}{2} a_{1 n}-\cos \frac{\psi}{2} a_{2 n}\right)^{2} \sim \Sigma_{2}(\psi):=\sum_{n \in \mathbb{Z}}\left(\sin ^{2} \frac{\psi}{2} b_{1 n}+\cos ^{2} \frac{\psi}{2} b_{2 n}\right) \times \\
\left(\sin \frac{\psi}{2} a_{1 n}-\cos \frac{\psi}{2} a_{2 n}\right)^{2}=\Sigma\left(C_{1}, C_{2}\right)=\sum_{n \in \mathbb{Z}}\left(C_{1}^{2} b_{1 n}+C_{2}^{2} b_{2 n}\right)\left(C_{1} a_{1 n}+C_{2} a_{2 n}\right)^{2} .
\end{gathered}
$$

\subsection{Some orthogonality problem in measure theory}

Our aim now is to find the minimal set of conditions of the orthogonality $\mu^{L_{t}} \perp \mu$ for all $t \in G L(2, \mathbb{R}) \backslash\{e\}$. To be more precise, consider more general situation.

Let $\alpha: G \rightarrow \operatorname{Aut}(X)$ be a measurable action of a group $G$ on a measurable space $(X, \mathfrak{B}, \mu)$ with the following property: $\mu^{\alpha_{t}} \perp \mu$ for all $t \in G \backslash\{e\}$. Consider a subset $G^{\perp}(\mu)$ in the group $G$ having the following property:

$$
\text { if } \mu^{\alpha_{t}} \perp \mu \forall t \in G^{\perp}(\mu) \text { then } \mu^{\alpha_{t}} \perp \mu \forall t \in G \backslash\{e\} .
$$

Problem. Find a minimal subset $G_{0}^{\perp}(\mu)$ having the property (4.5).

Example 4.1. Consider the nilpotent group $B(m, \mathbb{R})$ of upper triangular real $m \times m$ matrices with units on the diagonal acting on the space $X_{m}$ with the Gaussian product measure $\mu=\mu_{(b, a)}^{m}$, where $X_{m}$ and $\mu$ are defined as follows (see details in [17, 18]):

$$
X_{m}=\left\{I+\sum_{1 \leq k \leq m} \sum_{k<n} x_{k n} E_{k n}\right\}, \quad \mu^{m}=\otimes_{1 \leq k \leq m} \otimes_{k<n} \mu_{\left(b_{k n}, a_{k n}\right)} .
$$


Using results form [17] and [18] we conclude that the three following conditions are equivalent:

$$
\begin{array}{rcl}
(i) & \mu^{L_{t}} \perp \mu & \forall t \in B(m, \mathbb{R}) \backslash\{e\}, \\
\text { (ii) } & \mu^{L_{\exp \left(t E_{k n}\right)} \perp \mu} & \forall t \in \mathbb{R} \backslash\{0\}, \quad 1 \leq k<n \leq m, \\
\text { (iii) } & S_{k n}^{L}(\mu)=\infty & 1 \leq k<n \leq m,
\end{array}
$$

where $S_{k n}^{L}(\mu)$ is defined by (4.1)

$$
S_{k n}^{L}(\mu)=\sum_{r=n+1}^{\infty} \frac{b_{k r}}{2}\left(\frac{1}{2 b_{n r}}+a_{n r}^{2}\right) .
$$

In fact, it is sufficient to fix a nontrivial point $t_{k n} \neq 0$ on any subgroup $\exp \left(t E_{k n}\right)=I+t E_{k n}, t \in \mathbb{R}, 1 \leq k<n \leq m$. In this case the subset $G_{0}^{\perp}(\mu)$ is discrete and consists of $m(m-1) / 2$ points:

$$
G_{0}^{\perp}(\mu, t)=\left(I+t_{k n} E_{k n} \mid t_{k n} \in \mathbb{R} \backslash\{0\}, 1 \leq k<n \leq m\right),
$$

where $t=\left(t_{k n}\right)_{k n} \in(\mathbb{R} \backslash\{0\})^{m(m-1) / 2}$. For $t_{1} \neq t_{2} \in(\mathbb{R} \backslash\{0\})^{m(m-1) / 2}$ we get two distinct minimal subsets $G_{0}^{\perp}\left(\mu^{m}, t_{1}\right)$ and $G_{0}^{\perp}\left(\mu^{m}, t_{2}\right)$.

Example 4.2. Consider the solvable group $\operatorname{Bor}(m, \mathbb{R})$ of upper triangular real $m \times m$ matrices with nonzero elements on the diagonal acting on the space $X_{m}$ with the Gaussian product measure $\mu=\mu_{(b, a)}^{m}$, where $X_{m}$ and $\mu$ are defined as follows (see details in [1])

$$
X_{m}=\left\{x=\sum_{1 \leq k \leq m} \sum_{k \leq n} x_{k n} E_{k n}\right\}, \quad \mu_{(b, a)}^{m}=\otimes_{1 \leq k \leq m} \otimes_{k \leq n} \mu_{\left(b_{k n}, a_{k n}\right)} .
$$

Using [1, Theorem 5] we conclude that the following three conditions are equivalent:

$$
\begin{aligned}
& \mu^{L_{t}} \perp \mu, \quad \forall t \in \operatorname{Bor}(m, \mathbb{R}) \backslash\{e\}, \\
& \mu^{L_{\exp \left(t E_{k n}\right)}} \perp \mu \forall t \in \mathbb{R} \backslash\{0\}, \quad 1 \leq k<n \leq m, \\
& \mu^{L_{\exp \left(t E_{k n}\right) P_{k}}} \perp \mu \forall t \in \mathbb{R}, \quad 1 \leq k<n \leq m, \\
& \text { (iii) } S_{k n}^{L}(\mu)=\infty, \quad S_{k n}^{L,-}(\mu, t)=\infty, \quad 1 \leq k<n \leq m,
\end{aligned}
$$

where $S_{k n}^{L,-}(\mu, t)$ is defined by (4.2). As before, it is sufficient to fix a nontrivial point $t_{k n} \neq 0$ on any subgroup $\exp \left(t E_{k n}\right)=I+t E_{k n}, t \in \mathbb{R}$. But on 
the curves $\exp \left(t E_{k n}\right) P_{k}$ we can not omit any point $t \in \mathbb{R}$. Finally, a minimal subset depending on the choice of $t=\left(t_{k n}\right)_{k n} \in(\mathbb{R} \backslash\{0\})^{m(m-1) / 2}$ can be chosen as follows:

$$
\begin{gathered}
G_{0}^{\perp}(\mu, t)=\left(\exp \left(t_{k n} E_{k n}\right)=I+t_{k n} E_{k n} \mid t_{k n} \in \mathbb{R} \backslash\{0\}, 1 \leq k<n \leq m\right) \bigcup \\
\left(\exp \left(t E_{k n}\right) P_{k} \mid \forall t \in \mathbb{R}, 1 \leq k<n \leq m\right)
\end{gathered}
$$

where $P_{k}=I-2 E_{k k}$. For example, for $m=2$ we get $P_{1}=\operatorname{diag}(-1,1)$ and $P_{2}=\operatorname{diag}(1,-1)$.

Example 4.3. In the case of the group $G=\mathrm{GL}(2, \mathbb{R})$ acting on the space $X_{2}$ defined by (2.3) with the measure $\mu_{(b, a)}^{2}$ defined by (2.4) using Lemmas 4.1, we conclude that the description of the set $G_{0}^{\perp}\left(\mu_{(b, a)}^{2}\right)$ is as follows:

$$
\begin{gathered}
G_{0}^{\perp}\left(\mu_{(b, a)}^{2}, t_{12}, t_{21}\right)=\left(\exp \left(t_{12} E_{12}\right), \exp \left(t_{21} E_{21}\right) \mid t_{12}, t_{21} \in \mathbb{R} \backslash\{0\}\right) \bigcup \\
\left(\exp \left(t E_{12}\right) P_{1}, \exp \left(t E_{21}\right) P_{2} \mid \forall t \in \mathbb{R}\right) \bigcup\left(\tau_{-}(\phi, s) \mid \forall s>0, \phi \in[0,2 \pi)\right) .
\end{gathered}
$$

Remark 4.4. We note that except the one-parameter groups $E_{k n}(t)=I+$ $t E_{k n}, t \in \mathbb{R}$ all other element from the set $G_{0}^{\perp}(\mu)$ for $G=\operatorname{GL}(2, \mathbb{R})$ are of order 2, i.e., if $g \in\left\{\exp \left(t E_{k n}\right) P_{k}, \tau_{-}(\phi, s)\right\}$ then $g^{2}=e$.

\subsection{Approximation of $x_{k n}$ and $D_{k n}$}

We will formulate several lemmas, which will be useful for approximation of the independent variables $x_{k n}$ and operators $D_{k n}$ by combinations of the generators $A_{k n}$. For short, we shall write $A_{k n}$ instead of $A_{k n}^{R, 2}$.

In what follows we use the following notation for $f, g \in \mathbb{R}^{m}$

$$
\Delta(f, g)=\frac{\Gamma(f)+\Gamma(f, g)}{\Gamma(g)+1} .
$$

Lemma 4.2. For any $k, t \in \mathbb{Z}$ one has

$$
x_{1 n} x_{1 t} \in\left\langle A_{n k} A_{t k} \mathbf{1} \mid k \in \mathbb{Z}\right\rangle \Leftrightarrow \lim _{m} \Sigma_{1, m}(x, x)=\infty,
$$

where $\Sigma_{1, m}(x, x)=\Delta\left(f_{m}^{1}, g_{m}^{1}\right)$ and

$$
f_{m}^{1}=\left(\frac{b_{1 k}}{\sqrt{b_{1 k}^{2}+2 b_{1 k} b_{2 k}}}\right)_{k=-m}^{m}, \quad g_{m}^{1}=\left(\frac{b_{2 k}}{\sqrt{b_{1 k}^{2}+2 b_{1 k} b_{2 k}}}\right)_{k=-m}^{m} .
$$


Lemma 4.3. For any $k, t \in \mathbb{Z}$ we have

$$
x_{2 k} x_{2 t} \in\left\langle A_{k n} A_{t n} \mathbf{1} \mid n \in \mathbb{Z}\right\rangle \Leftrightarrow \lim _{m} \Sigma_{2, m}(x, x)=\infty,
$$

where $\Sigma_{2, m}(x, x)=\Delta\left(f_{m}^{2}, g_{m}^{2}\right)$ and

$$
f_{m}^{2}=\left(\frac{b_{2 k}}{\sqrt{b_{2 k}^{2}+2 b_{1 k} b_{2 k}}}\right)_{k=-m}^{m}, \quad g_{m}^{2}=\left(\frac{b_{1 k}}{\sqrt{b_{2 k}^{2}+2 b_{1 k} b_{2 k}}}\right)_{k=-m}^{m} .
$$

Remark 4.5. We say that two series $\sum_{n} a_{n}$ and $\sum_{n} b_{n}$ with positive $a_{n}, b_{n}$ are equivalent if they are simultaneously convergent or divergent. In this case we shall use the notaions $\sum_{n} a_{n} \sim \sum_{n} b_{n}$. Using the obvious equivalence of the following two series with positive $a_{n}$ and $b_{n}$

$$
\sum_{n \in \mathbb{N}} \frac{a_{n}}{a_{n}+b_{n}} \sim \sum_{n \in \mathbb{N}} \frac{a_{n}}{b_{n}}
$$

we have the following estimation (we set $\Sigma^{12}=\sum_{k \in \mathbb{Z}} \frac{b_{1 k}}{b_{2 k}}$ and $\Sigma^{21}=\sum_{k \in \mathbb{Z}} \frac{b_{2 k}}{b_{1 k}}$ )

$$
\begin{aligned}
& \left\|f^{1}\right\|^{2}=\sum_{k \in \mathbb{Z}} \frac{b_{1 k}^{2}}{b_{1 k}^{2}+2 b_{1 k} b_{2 k}} \sim \sum_{k \in \mathbb{Z}} \frac{b_{1 k}}{2 b_{2 k}}=\frac{\Sigma^{12}}{2}, \\
& \left\|f^{2}\right\|^{2}=\sum_{k \in \mathbb{Z}} \frac{b_{2 k}^{2}}{b_{2 k}^{2}+2 b_{1 k} b_{2 k}} \sim \sum_{k \in \mathbb{Z}} \frac{b_{2 k}}{2 b_{1 k}}=\frac{\Sigma^{21}}{2}, \\
& \left\|g^{1}\right\|^{2}=\sum_{k \in \mathbb{Z}} \frac{b_{2 k}^{2}}{b_{1 k}^{2}+2 b_{1 k} b_{2 k}}<\sum_{k \in \mathbb{Z}} \frac{b_{2 k}}{2 b_{1 k}}=\frac{\Sigma^{21}}{2}, \\
& \left\|g^{1}\right\|^{2}=\sum_{k \in \mathbb{Z}} \frac{b_{1 k}^{2}}{b_{2 k}^{2}+2 b_{1 k} b_{2 k}}<\sum_{k \in \mathbb{Z}} \frac{b_{1 k}}{2 b_{2 k}}=\frac{\Sigma^{12}}{2},
\end{aligned}
$$

we conclude that $\lim _{m} \Sigma_{1, m}(x, x)=\infty$ if

$$
\lim _{m} \Sigma_{1, m}^{\prime}(x, x):=\lim _{m}\left(\sum_{k=-m}^{m} \frac{b_{1 k}}{b_{2 k}}\right)\left(\sum_{k=-m}^{m} \frac{b_{2 k}}{b_{1 k}}\right)^{-1}=\Sigma^{12} / \Sigma^{21}=\infty
$$

and $\lim _{m} \Sigma_{2, m}(x, x)=\infty$ if

$$
\lim _{m} \Sigma_{2, m}^{\prime}(x, x):=\lim _{m}\left(\sum_{k=-m}^{m} \frac{b_{2 k}}{b_{1 k}}\right)\left(\sum_{k=-m}^{m} \frac{b_{1 k}}{b_{2 k}}\right)^{-1}=\Sigma^{21} / \Sigma^{12}=\infty .
$$


Lemma 4.4. For any $n \in \mathbb{Z}$ we have

$$
D_{1 n} \mathbf{1} \in\left\langle A_{k n} \mathbf{1} \mid k \in \mathbb{Z}\right\rangle \quad \Leftrightarrow \quad \lim _{m} \Sigma_{1, m}(D)=\infty,
$$

where $\Sigma_{1, m}(D)=\Delta\left(f_{m}, g_{m}\right)$ and

$$
f_{m}=\left(a_{1 k}\left(\frac{1}{2 b_{1 k}}+\frac{1}{2 b_{2 k}}\right)^{-1 / 2}\right)_{k=-m}^{m}, g_{m}=\left(a_{2 k}\left(\frac{1}{2 b_{1 k}}+\frac{1}{2 b_{2 k}}\right)^{-1 / 2}\right)_{k=-m}^{m}
$$

Lemma 4.5. Set $\Sigma_{2, m}(D)=\Delta\left(g_{m}, f_{m}\right)$. For any $n \in \mathbb{Z}$ we get

$$
D_{2 n} \mathbf{1} \in\left\langle A_{k n} \mathbf{1} \mid k \in \mathbb{Z}\right\rangle \quad \Leftrightarrow \quad \lim _{m} \Sigma_{2, m}(D)=\infty .
$$

Lemma 4.6. For any $k \in \mathbb{Z}$ we get

$$
x_{1 k} \mathbf{1} \in\left\langle D_{1 n} A_{k n} \mathbf{1} \mid n \in \mathbb{Z}\right\rangle \quad \Leftrightarrow \quad \sum_{n \in \mathbb{Z}} \frac{b_{1 n}}{b_{2 n}}=\infty .
$$

Lemma 4.7. For any $k \in \mathbb{Z}$ we have

$$
x_{2 k} \mathbf{1} \in\left\langle D_{2 n} A_{k n} \mathbf{1} \mid n \in \mathbb{Z}\right\rangle \quad \Leftrightarrow \quad \sum_{n \in \mathbb{Z}} \frac{b_{2 n}}{b_{1 n}}=\infty .
$$

Our aim now is to show that some of the expressions $\Sigma_{1, m}(x, x), \Sigma_{2, m}(x, x)$ and $\Sigma_{1, m}(D), \Sigma_{2, m}(D)$ tend to infinity if $\mu^{L_{t}} \perp \mu$ for all $t \in G L(2, \mathbb{R}) \backslash\{e\}$.

Let $\Gamma\left(f_{1}, f_{2}, \ldots, f_{n}\right)$ be the Gramm determinant and $\gamma\left(f_{1}, f_{2}, \ldots, f_{n}\right)$ be the Gramm matrix of $n$ vectors $f_{1}, f_{2}, \ldots, f_{n}$ in a Hilbert space (see [7]). The following lemma is trivial and well known but we need exact formulas.

Lemma 4.8. Let $f_{1}, f_{2}$ be two vectors in a Hilbert space. The distance $\delta\left\langle f_{2}, f_{1}\right\rangle$ of the vector $f_{2}$ from the line $\left\langle f_{1}\right\rangle$ generated by $f_{1}$ is given by the following formula:

$$
\delta^{2}\left\langle f_{2}, f_{1}\right\rangle=\left\|f_{2}-\frac{\left(f_{2}, f_{1}\right)}{\left(f_{1}, f_{1}\right)} f_{1}\right\|^{2}=\frac{\Gamma\left(f_{1}, f_{2}\right)}{\Gamma\left(f_{1}\right)} .
$$

Proof. Obviously, $\delta^{2}\left\langle f_{2}, f_{1}\right\rangle=\left\|f_{2}-f_{0}\right\|^{2}$ where $f_{0}=C_{1} f_{1}$ such that $\left(f_{2}-f_{0}, f_{1}\right)=0$. We have

$$
0=\left(f_{2}-f_{0}, f_{1}\right)=\left(f_{2}, f_{1}\right)-C_{1}\left(f_{1}, f_{1}\right) \text { hence, } \quad C_{1}=\frac{\left(f_{2}, f_{1}\right)}{\left(f_{1}, f_{1}\right)} .
$$


Finally,

$$
\begin{array}{r}
\delta^{2}\left\langle f_{2}, f_{1}\right\rangle=\left\|f_{2}-f_{0}\right\|^{2}=\left\|f_{2}-C_{1} f_{1}\right\|^{2}=\left(f_{2}, f_{2}\right)-2 C_{1}\left(f_{2}, f_{1}\right)+C_{1}^{2}\left(f_{1}, f_{1}\right)= \\
\left(f_{2}, f_{2}\right)-\frac{2\left(f_{2}, f_{1}\right)\left(f_{2}, f_{1}\right)}{\left(f_{1}, f_{1}\right)}+\frac{\left(f_{2}, f_{1}\right)^{2}}{\left(f_{1}, f_{1}\right)^{2}}\left(f_{1}, f_{1}\right)= \\
\frac{\left(f_{2}, f_{2}\right)\left(f_{1}, f_{1}\right)-\left(f_{2}, f_{1}\right)\left(f_{1}, f_{2}\right)}{\left(f_{1}, f_{1}\right)}=\frac{\Gamma\left(f_{1}, f_{2}\right)}{\Gamma\left(f_{1}\right)} .
\end{array}
$$

Lemma 4.9. Let $f=\left(f_{k}\right)_{k \in \mathbb{N}}$ and $g=\left(g_{k}\right)_{k \in \mathbb{N}}$ be two real vectors such that $\|f\|^{2}=\infty$ where $\|f\|^{2}=\sum_{k} f_{k}^{2}$. Denote by $f_{(n)}, g_{(n)} \in \mathbb{R}^{n}$ their projections to the subspace $\mathbb{R}^{n}$, i.e., $f_{(n)}=\left(f_{k}\right)_{k=1}^{n}, \quad g_{(n)}=\left(g_{k}\right)_{k=1}^{n}$ and set

$$
\Delta\left(f_{(n)}, g_{(n)}\right)=\frac{\Gamma\left(f_{(n)}\right)+\Gamma\left(f_{(n)}, g_{(n)}\right)}{\Gamma\left(g_{(n)}\right)+1} \text { then } \lim _{n \rightarrow \infty} \Delta\left(f_{(n)}, g_{(n)}\right)=\infty
$$

in the following cases:

$$
\|g\|^{2}<\infty
$$

(c) $\|f\|^{2}=\|g\|^{2}=\|f+s g\|^{2}=\infty$, for all $s \in \mathbb{R} \backslash\{0\}$.

Proof. Obviously $\lim _{n \rightarrow \infty} \Delta\left(f_{(n)}, g_{(n)}\right)=\infty$ if conditions (a) or (b) hold. The implication $(c) \Rightarrow(4.12)$ is based on the following lemma.

Lemma 4.10. Let $f=\left(f_{k}\right)_{k \in \mathbb{N}}$ and $g=\left(g_{k}\right)_{k \in \mathbb{N}}$ be two real vectors such that

$$
\begin{gathered}
\|f\|^{2}=\|g\|^{2}=\left\|C_{1} f+C_{2} g\right\|^{2}=\infty, \quad \text { for all } \quad\left(C_{1}, C_{2}\right) \in \mathbb{R}^{2} \backslash\{0\} \\
\text { then } \quad \lim _{n \rightarrow \infty} \frac{\Gamma\left(f_{(n)}, g_{(n)}\right)}{\Gamma\left(g_{(n)}\right)}=\infty \quad \text { and } \quad \lim _{n \rightarrow \infty} \frac{\Gamma\left(f_{(n)}, g_{(n)}\right)}{\Gamma\left(f_{(n)}\right)}=\infty
\end{gathered}
$$

Proof. Assume that $\frac{\left\|f_{(n)}\right\|}{\left\|g_{(n)}\right\|} \leq C_{1}, \forall n \in \mathbb{N}$. The case $\frac{\left\|f_{(n)}\right\|}{\left\|g_{(n)}\right\|} \geq C_{1}$ is similar. In this case $\frac{\Gamma\left(f_{(n)}, g_{(n)}\right)}{\Gamma\left(g_{(n)}\right)} \leq C_{1}^{2} \frac{\Gamma\left(f_{(n)}, g_{(n)}\right)}{\Gamma\left(f_{(n)}\right)}$ therefore, to prove (4.14) it is sufficient to prove that $\lim _{n \rightarrow \infty} \frac{\Gamma\left(f_{(n)}, g_{(n)}\right)}{\Gamma\left(g_{(n)}\right)}=\infty$. Let us suppose the opposite, i.e., that for all $n \in \mathbb{N}$ holds

$$
\frac{\Gamma\left(f_{(n)}, g_{(n)}\right)}{\Gamma\left(g_{(n)}\right)} \leq C
$$


Set $t_{n}=\frac{\left\|f_{(n)}\right\|}{\left\|g_{(n)}\right\|}$ then by the inequality $\frac{\left\|f_{(n)}\right\|}{\left\|g_{(n)}\right\|} \leq C_{1}$ there exists a subsequence $t_{n_{k}}$ such that the limit exists

$$
\lim _{k \rightarrow \infty} t_{n_{k}}=t_{0} \in\left[0, C_{1}\right]
$$

Let $\alpha_{n}$ be an angle between two vectors $f_{(n)}, g_{(n)} \in \mathbb{R}^{n}$. Since $\frac{\Gamma(f, g)}{\Gamma(g)}$ is the square of the distance of the vector $f$ from the line generated by $g$ by Lemma 4.8, we have

$$
\frac{\Gamma\left(f_{(n)}, g_{(n)}\right)}{\Gamma\left(g_{(n)}\right)}=\left\|f_{(n)}\right\|^{2} \sin ^{2} \alpha_{n} \leq C, \quad \text { therefore } \quad \alpha_{n} \sim\left\|f_{n}\right\|^{-1} \rightarrow 0
$$

For $k, n \in \mathbb{N}$ set $M(k, n)=\left|\begin{array}{ll}f_{k} & f_{n} \\ g_{k} & g_{n}\end{array}\right|$, then by the Lagrange identity for $f_{(m)}=\left(f_{k}\right)_{k=1}^{m}, g_{(m)}=\left(g_{k}\right)_{k=1}^{m} \in \mathbb{R}^{m}([3]$, Ch.11, $\S 6$, formulae $\left.(7)]\right)$ we have

$$
\Gamma\left(f_{(m)}, g_{(m)}\right)=\sum_{k<n \leq m} M^{2}(k, n)
$$

therefore, the inequality (4.15) will have the following form

$$
\frac{\Gamma\left(f_{(m)}, g_{(m)}\right)}{\Gamma\left(g_{(m)}\right)}=\frac{\sum_{k<n \leq m} M^{2}(k, n)}{\sum_{k=1}^{m} g_{k}^{2}} \leq C, \quad m \in \mathbb{N} .
$$

For $t \in \mathbb{R}$ and $f_{(n)}, g_{(n)} \in \mathbb{R}^{n}$ introduce the function

$$
F_{n}(t)=\left\|f_{(n)}-t g_{(n)}\right\|^{2}=\left(f_{(n)}, f_{(n)}\right)-2 t\left(f_{(n)}, g_{(n)}\right)+t^{2}\left(g_{(n)}, g_{(n)}\right) .
$$

The minimum of the function $F_{n}(t)$ is reached at $t_{0}^{(n)}=\frac{\left(f_{(n)}, g_{(n)}\right)}{\left(g_{(n)}, g_{(n)}\right)}$ therefore, we have

$$
F_{n}(t)=\left(g_{(n)}, g_{(n)}\right)\left(t-t_{0}^{(n)}\right)^{2}+\frac{\Gamma\left(f_{(n)}, g_{(n)}\right)}{\Gamma\left(g_{(n)}\right)}, \quad F_{n}\left(t_{0}^{(n)}\right)=\frac{\Gamma\left(f_{(n)}, g_{(n)}\right)}{\Gamma\left(g_{(n)}\right)},
$$

hence,

$$
F_{n}\left(t_{0}\right)-F_{n}\left(t_{0}^{(n)}\right)=\left(g_{(n)}, g_{(n)}\right)\left(t_{0}-t_{0}^{(n)}\right)^{2} .
$$

Since $F_{n}\left(t_{0}^{(n)}\right)=\frac{\Gamma\left(f_{(n)}, g_{(n)}\right)}{\Gamma\left(g_{(n)}\right)}$ is bounded by assumption and

$$
\lim _{n \rightarrow \infty} F_{n}(t)=\lim _{n \rightarrow \infty}\left\|f_{(n)}-t g_{(n)}\right\|^{2}=\infty \quad \text { for all } t \in \mathbb{R},
$$


by the condition (4.13), we conclude that $\lim _{n \rightarrow \infty}\left(F_{n}\left(t_{0}\right)-F_{n}\left(t_{0}^{n}\right)\right)=\infty$.

We show that condition (4.15) implies that $F_{n}\left(t_{0}\right)-F_{n}\left(t_{0}^{(n)}\right)$ is bounded. This contradiction will prove the lemma. Indeed, we have

$$
t_{0}^{(n+1)}-t_{0}^{(n)}=\frac{\left(f_{(n+1)}, g_{(n+1)}\right)}{\left(g_{(n+1)}, g_{(n+1)}\right)}-\frac{\left(f_{(n)}, g_{(n)}\right)}{\left(g_{(n)}, g_{(n)}\right)}=-\frac{\sum_{k=1}^{n} M(k, n+1) g_{k} g_{n+1}}{\left(g_{(n)}, g_{(n)}\right)\left(g_{(n+1)}, g_{(n+1)}\right)}
$$

and

$$
\begin{gathered}
t_{0}^{(n+m)}-t_{0}^{(n)}=\frac{\left(f_{(n+m)}, g_{(n+m)}\right)}{\left(g_{(n+m)}, g_{(n+m)}\right)}-\frac{\left(f_{(n)}, g_{(n)}\right)}{\left(g_{(n)}, g_{(n)}\right)} \\
=-\frac{\sum_{k=1}^{n} \sum_{r=n+1}^{n+m} M(k, r) g_{k} g_{r}}{\left(g_{(n)}, g_{(n)}\right)\left(g_{(n+m)}, g_{(n+m)}\right)}=-\frac{\left(M_{n, m} g^{n, m}, g_{(n)}\right)}{\left(g_{(n)}, g_{(n)}\right)\left(g_{(n+m)}, g_{(n+m)}\right)}
\end{gathered}
$$

where the vector $g^{n, m} \in \mathbb{R}^{m}$ and the rectangular matrix $M_{n, m} \in \operatorname{Mat}(\mathbb{R}, n \times$ $m$ ) are defined as follows:

$g^{n, m}=\left(g_{k}\right)_{k=n+1}^{n+m} \quad$ and $\quad M_{n, m}=(M(k, r))_{k, r} \quad 1 \leq k \leq n, \quad n+1 \leq r \leq n+m$.

We observe that $\lim _{n} t_{0}^{(n)}=\lim _{n} t_{n}=t_{0}$. Indeed, if $n \rightarrow \infty$ by (4.16) we have

$$
t_{0}^{(n)}=\frac{\left(f_{(n)}, g_{(n)}\right)}{\left(g_{(n)}, g_{(n)}\right)}=\frac{\left\|f_{(n)}\right\|\left\|g_{(n)}\right\| \cos \alpha_{n}}{\left\|g_{(n)}\right\|^{2}}=t_{n} \cos \alpha_{n} \rightarrow t_{0} .
$$

Finally, for all $n, m \in \mathbb{N}$ we get by (4.18), (4.19) and the Schwartz inequality

$$
\begin{gathered}
F_{n}\left(t_{0}^{(n+m)}\right)-F_{n}\left(t_{0}^{(n)}\right)=\left(g_{(n)}, g_{(n)}\right)\left(t_{0}^{(n+m)}-t_{0}^{(n)}\right)^{2}= \\
\left(g_{(n)}, g_{(n)}\right)\left[\frac{\left(M_{n, m} g^{n, m}, g_{(n)}\right)}{\left(g_{(n)}, g_{(n)}\right)\left(g_{(n+m)}, g_{(n+m)}\right)}\right]^{2} \leq \\
\frac{\left\|g_{(n)}\right\|^{2}\left\|M_{n, m} g^{n, m}\right\|^{2}\left\|g_{(n)}\right\|^{2}}{\left\|g_{n}(n)\right\|^{4}\left\|g_{(n+m)}\right\|^{4}} \leq \frac{\left\|M_{n, m}\right\|_{\sigma_{2}}^{2}\left\|g^{n, m}\right\|^{2}}{\left\|g_{(n+m)}\right\|^{4}} \leq \frac{\left\|M_{n+m}\right\|_{\sigma_{2}}^{2}}{\left\|g_{(n+m)}\right\|^{2}} \leq C,
\end{gathered}
$$

where $M_{m}:=(M(k, r))_{k<r \leq m}$ and

$$
\left\|M_{n, m}\right\|_{\sigma_{2}}^{2}=\sum_{k=1}^{n} \sum_{r=n+1}^{n+m} M^{2}(k, r), \quad\left\|M_{m}\right\|_{\sigma_{2}}^{2}=\sum_{k<r \leq m} M^{2}(k, r)=\Gamma\left(f_{(m)}, g_{(m)}\right) .
$$

Fix $\varepsilon>0$. Since $\lim _{m} t_{0}^{(m)}=t_{0}$ and the functions $F_{n}(t)$ are continuous we conclude that there exists $m_{n} \geq n$ such that $F_{n}\left(t_{0}^{(m)}\right)>F_{n}\left(t_{0}\right)-\varepsilon, \forall m \geq m_{n}$, 
in particular, $F_{n}\left(t_{0}^{\left(m_{n}\right)}\right)>F_{n}\left(t_{0}\right)-\varepsilon$. Since $\lim _{n} F_{n}\left(t_{0}\right)=\infty$ we conclude that

$$
\lim _{n} F_{n}\left(t_{0}^{\left(m_{n}\right)}\right) \geq \lim _{n}\left(F_{n}\left(t_{0}\right)-\varepsilon\right)=\infty
$$

that contradicts the condition $F_{n}\left(t_{0}^{(n+m)}\right)-F_{n}\left(t_{0}^{(n)}\right) \leq C$ for all $m, n \in \mathbb{N}$.

Lemma 4.11. If $\mu^{L_{t}} \perp \mu$ for all $t \in G L(2, \mathbb{R}) \backslash\{e\}$, we can approximate one of the following pair of operators: $\left(x_{1 n}, x_{2 n}\right),\left(x_{1 n}, D_{2 n}\right),\left(D_{1 n}, x_{2 n}\right)$, or $\left(D_{1 n}, D_{2 n}\right)$.

PROOF. For the convenience of the readers we collect the important formulas below:

$$
\begin{aligned}
& \Sigma_{1, m}(x, x)=\frac{\Gamma\left(f_{m}^{1}\right)+\Gamma\left(f_{m}^{1}, g_{m}^{1}\right)}{\Gamma\left(g_{m}^{1}\right)+1}=\frac{\sum_{k=-m}^{m} \frac{b_{1 k}^{2}}{b_{1 k}^{2}+2 b_{1 k} b_{2 k}}+\Gamma\left(f_{m}^{1}, g_{m}^{1}\right)}{\sum_{k=-m}^{m} \frac{b_{2 k}^{2}}{b_{1 k}^{2}+2 b_{1 k} b_{2 k}}+1} \\
& \Sigma_{2, m}(x, x)=\frac{\Gamma\left(f_{m}^{2}\right)+\Gamma\left(f_{m}^{2}, g_{m}^{2}\right)}{\Gamma\left(g_{m}^{2}\right)+1}=\frac{\sum_{k=-m}^{m} \frac{b_{2 k}^{2}}{b_{2 k}^{2}+2 b_{1 k} b_{2 k}}+\Gamma\left(f_{m}^{2}, g_{m}^{2}\right)}{\sum_{k=-m}^{m} \frac{b_{1 k}^{2}}{b_{2 k}^{2}+2 b_{1 k} b_{2 k}}+1} \\
& \Sigma_{1, m}(D)=\frac{\Gamma\left(f_{m}\right)+\Gamma\left(f_{m}, g_{m}\right)}{\Gamma\left(g_{m}\right)+1}=\frac{\sum_{k=-m}^{m} \frac{a_{1 k}^{2}}{\frac{1}{2 b_{1 k}}+\frac{1}{2 b_{2 k}}}+\Gamma\left(f_{m}, g_{m}\right)}{\sum_{k=-m}^{m} \frac{a_{2 k}^{2}}{\frac{1}{2 b_{1 k}}+\frac{1}{2 b_{2 k}}}+1}
\end{aligned}
$$

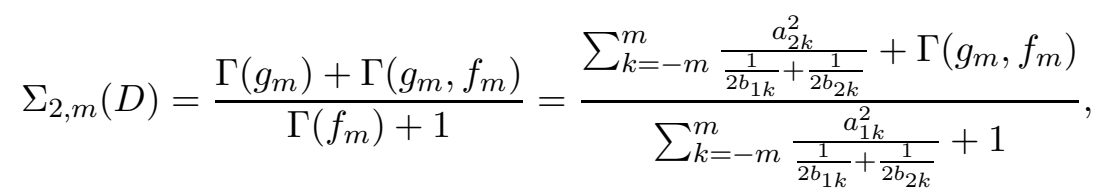

$$
\begin{aligned}
& f_{m}^{1}=\left(\frac{b_{1 k}}{\sqrt{b_{1 k}^{2}+2 b_{1 k} b_{2 k}}}\right)_{k=-m}^{m}, \quad g_{m}^{1}=\left(\frac{b_{2 k}}{\sqrt{b_{1 k}^{2}+2 b_{1 k} b_{2 k}}}\right)_{k=-m}^{m}, \\
& f_{m}^{2}=\left(\frac{b_{2 k}}{\sqrt{b_{2 k}^{2}+2 b_{1 k} b_{2 k}}}\right)_{k=-m}^{m}, \quad g_{m}^{2}=\left(\frac{b_{1 k}}{\sqrt{b_{2 k}^{2}+2 b_{1 k} b_{2 k}}}\right)_{k=-m}^{m}, \\
& f_{m}=\left(a_{1 k}\left(\frac{1}{2 b_{1 k}}+\frac{1}{2 b_{2 k}}\right)^{-1 / 2}\right)_{k=-m}^{m}, \quad g_{m}=\left(a_{2 k}\left(\frac{1}{2 b_{1 k}}+\frac{1}{2 b_{2 k}}\right)^{-1 / 2}\right)_{k=-m}^{m} .
\end{aligned}
$$


To estimate $\Sigma_{1, m}(x, x)$ and $\Sigma_{2, m}(x, x)$ consider three possibilities:

(1) $\Sigma^{12}:=\sum_{k \in \mathbb{Z}} \frac{b_{1 k}}{b_{2 k}}<\infty$, (2) $\Sigma^{21}:=\sum_{k \in \mathbb{Z}} \frac{b_{2 k}}{b_{1 k}}<\infty, \quad$ (3) $\sum_{k \in \mathbb{Z}} \frac{b_{1 k}}{b_{2 k}}=\sum_{k \in \mathbb{Z}} \frac{b_{2 k}}{b_{1 k}}=\infty$.

We present the results in the table I.

\begin{tabular}{|l|l|l|l|l|l|}
\hline table I & $(1)$ & $(2)$ & $(3 \mathrm{a})$ & $(3 \mathrm{~b})$ & $(3 \mathrm{c})$ \\
\hline$\Sigma^{12}$ & $<\infty$ & & $\infty$ & $\infty$ & $\infty$ \\
\hline$\Sigma^{21}$ & & $<\infty$ & $\infty$ & $\infty$ & $\infty$ \\
\hline$\left\|g^{1}\right\|$ & & & $<\infty$ & & \\
\hline$\left\|g^{2}\right\|$ & & & & $<\infty$ & \\
\hline Lemma & $4.3,[4.4$, & $4.2,[4.4$, & 4.2, & 4.3, & $4.2,4.3$, \\
& $4.5,[4.10$ & $4.5,4.10$ & & & $4.10,4.13$ \\
\hline & $x_{2 n}, D_{1 n}, D_{2 n}$ & $x_{1 n}, D_{1 n}, D_{2 n}$ & $x_{1 n}, x_{2 n}$ & $x_{1 n}, x_{2 n}$ & $x_{1 n}, x_{2 n}$ \\
\hline
\end{tabular}

Case (1). If $\Sigma^{12}<\infty$ then $\Sigma^{21}=\infty$ and we have $\lim _{m \rightarrow \infty} \Sigma_{2, m}(x, x)=$ $\infty$ by Remark 4.5. Hence, $x_{2 n} x_{2 t} \eta \mathfrak{A}$, by Lemma 4.3 and $x_{2 n} \eta \mathfrak{A}$, by Lemma 3.3. We can approximate $D_{1 n}$ and $D_{2 n}$ by Lemmas 4.4, 4.5 and Lemma 4.10.

$D_{1 n} \eta \mathfrak{A} \quad$ if $\frac{\Gamma\left(f_{m}\right)+\Gamma\left(f_{m}, g_{m}\right)}{\Gamma\left(g_{m}\right)+1} \rightarrow \infty, D_{2 n} \eta \mathfrak{A} \quad$ if $\frac{\Gamma\left(g_{m}\right)+\Gamma\left(g_{m}, f_{m}\right)}{\Gamma\left(f_{m}\right)+1} \rightarrow \infty$,

where $f_{m}$ and $g_{m}$ are defined by (4.26). Set

$$
f=\left(a_{1 k}\left(\frac{1}{2 b_{1 k}}+\frac{1}{2 b_{2 k}}\right)^{-1 / 2}\right)_{k \in \mathbb{Z}}, \quad g=\left(a_{2 k}\left(\frac{1}{2 b_{1 k}}+\frac{1}{2 b_{2 k}}\right)^{-1 / 2}\right)_{k \in \mathbb{Z}} .
$$

Since $\sum_{k \in \mathbb{Z}} \frac{b_{1 k}}{b_{2 k}}<\infty$, we conclude that

$$
\|f\|^{2}=\|g\|^{2}=\|f-s g\|^{2}=\infty
$$

Indeed, we have

$$
\begin{gathered}
\|f\|^{2}=\sum_{k \in \mathbb{Z}} \frac{a_{1 k}^{2}}{\frac{1}{2 b_{1 k}}+\frac{1}{2 b_{2 k}}}=\sum_{k \in \mathbb{Z}} \frac{b_{1 k} a_{1 k}^{2}}{\frac{1}{2}+\frac{b_{1 k}}{2 b_{2 k}}} \sim 2 \sum_{k \in \mathbb{Z}} b_{1 k} a_{1 k}^{2}=S_{11}^{L}(\mu)=\infty, \\
\|g\|^{2}=\sum_{k \in \mathbb{Z}} \frac{b_{1 k} a_{2 k}^{2}}{\frac{1}{2}+\frac{b_{1 k}}{2 b_{2 k}}} \sim \sum_{k \in \mathbb{Z}} b_{1 k} a_{2 k}^{2} \sim \sum_{k \in \mathbb{Z}} \frac{b_{1 k}}{2}\left(\frac{1}{2 b_{2 k}}+a_{2 k}^{2}\right)=S_{12}^{L}(\mu)=\infty,
\end{gathered}
$$




$$
\begin{gathered}
\|f-s g\|^{2}=\sum_{k \in \mathbb{Z}} \frac{b_{1 k}\left(a_{1 k}-s a_{2 k}\right)^{2}}{\frac{1}{2}+\frac{b_{1 k}}{2 b_{2 k}}} \sim \sum_{k \in \mathbb{Z}} b_{1 k}\left(a_{1 k}-s a_{2 k}\right)^{2}=\frac{1}{4} \sum_{k \in \mathbb{Z}} b_{1 k}\left(-2 a_{1 k}+2 s a_{2 k}\right)^{2} \\
\sim \frac{1}{2}\left(\frac{(2 s)^{2}}{4} \sum_{k \in \mathbb{Z}} \frac{b_{1 k}}{b_{2 k}}+\sum_{k \in \mathbb{Z}} \frac{b_{1 k}}{2}\left(-2 a_{1 k}+2 s a_{2 k}\right)^{2}\right)=\frac{1}{2} S_{12}^{L,-}(\mu, t)=\infty,
\end{gathered}
$$

for $t=2 s($ see (4.2) $)$. Therefore, by Lemma 4.10 we conclude (see(4.14) ) that $\lim _{n \rightarrow \infty} \frac{\Gamma\left(f_{(n)}, g_{(n)}\right)}{\Gamma\left(g_{(n)}\right)}=\infty$ and $\lim _{n \rightarrow \infty} \frac{\Gamma\left(f_{(n)}, g_{(n)}\right)}{\Gamma\left(f_{(n)}\right)}=\infty$, so $D_{1 n}, D_{2 n} \eta \mathfrak{A}$ by Lemmas 4.4 and 4.5. Finally, $x_{2 n} D_{1 n} D_{2 n} \eta \mathfrak{A}$. Now we get $A_{k n}-x_{2 k} D_{2 n}=$ $x_{1 k} D_{1 n}, k, n \in \mathbb{Z}$ and the proof is complete since we are in the case $m=1$.

Case (2). If $\Sigma^{21}<\infty$ then $\Sigma^{12}=\infty$ and we have $\lim _{m \rightarrow \infty} \Sigma_{1, m}(x, x)=$ $\infty$, by Remark 4.5. Hence, $x_{1 n} x_{1 t} \eta \mathfrak{A}$, by Lemma 4.2 and $x_{1 n} \eta \mathfrak{A}$, by Lemma 3.3 . As in the previous case, the condition $\sum_{k \in \mathbb{Z}} \frac{b_{2 k}}{b_{1 k}}<\infty$ implies

$$
\|f\|^{2} \sim S_{21}^{L}(\mu)=\infty, \quad\|g\|^{2} \sim S_{22}^{L}(\mu)=\infty, \quad\|f-s g\|^{2} \sim S_{21}^{L,-}(\mu, t)=\infty,
$$

for $t=\frac{2}{s}$. Exactly, as in the case (1), we can approximate $D_{1 n}$ and $D_{2 n}$. Finally, $x_{1 n} D_{1 n} D_{2 n} \eta \mathfrak{A}$. Further, $A_{k n}-x_{1 k} D_{1 n}=x_{2 k} D_{2 n}, k, n \in \mathbb{Z}$ and the proof is complete.

Case (3). Let $\sum_{k \in \mathbb{Z}} \frac{b_{1 k}}{b_{2 k}}=\sum_{k \in \mathbb{Z}} \frac{b_{2 k}}{b_{1 k}}=\infty$. Set $c_{n}=\frac{b_{2 n}}{b_{1 n}}, n \in \mathbb{Z}$. The vectors $f_{m}^{1}, g_{m}^{1}, f_{m}^{2}, g_{m}^{2}$ are defined as follows (see (4.24) and (4.25)):

$$
\begin{aligned}
f_{m}^{1} & =\left(\frac{1}{\sqrt{1+2 c_{n}}}\right)_{-m}^{m}, & g_{m}^{1} & =\left(\frac{c_{n}}{\sqrt{1+2 c_{n}}}\right)_{-m}^{m}, \\
f_{m}^{2} & =\left(\sqrt{\frac{c_{n}}{c_{n}+2}}\right)_{-m}^{m}, & g_{m}^{2} & =\left(\frac{1}{\sqrt{c_{n}^{2}+2 c_{n}}}\right)_{-m}^{m} .
\end{aligned}
$$

We show that

$$
\left\|f^{1}\right\|^{2}=\left\|f^{2}\right\|^{2}=\left\|g^{1}\right\|^{2}+\left\|g^{2}\right\|^{2}=\infty
$$

Indeed, we have

$$
\begin{aligned}
& \left\|f^{1}\right\|^{2}=\sum_{n \in \mathbb{Z}}\left(1+2 c_{n}\right)^{-1} \sim \sum_{n \in \mathbb{Z}} c_{n}^{-1}=\Sigma^{12}=\infty, \\
& \left\|f^{2}\right\|^{2}=\sum_{n \in \mathbb{Z}} c_{n}\left(c_{n}+2\right)^{-1} \sim \sum_{n \in \mathbb{Z}} c_{n}=\Sigma^{21}=\infty .
\end{aligned}
$$

Let us suppose that $\left\|g^{1}\right\|^{2}+\left\|g^{2}\right\|^{2}<\infty$ then

$$
\infty>\left\|g^{1}\right\|^{2}+\left\|g^{2}\right\|^{2}=\sum_{n \in \mathbb{Z}}\left(\frac{c_{n}^{2}}{1+2 c_{n}}+\frac{1}{c_{n}^{2}+2 c_{n}}\right)>\sum_{n \in \mathbb{Z}} \frac{1+c_{n}^{2}}{\left(1+c_{n}\right)^{2}},
$$


hence, $\sum_{n \in \mathbb{Z}} \frac{1}{\left(1+c_{n}\right)^{2}}<\infty$ and $\sum_{n \in \mathbb{Z}} \frac{c_{n}^{2}}{\left(1+c_{n}\right)^{2}}<\infty$ therefore,

$$
\infty>\sum_{n \in \mathbb{Z}} \frac{\left(1+c_{n}\right)^{2}}{\left(1+c_{n}\right)^{2}}=\sum_{n \in \mathbb{Z}} 1=\infty
$$

This contradiction proves that $\left\|g^{1}\right\|^{2}+\left\|g^{2}\right\|^{2}=\infty$. We shall come back to the case $I(3)$ later. We show that in the case $A$ (see 4.38) ) we can approximate $x_{1 n}$ and $x_{2 n}$.

Now we study the possibility of the approximation of $D_{1 n}$ and $D_{2 n}$ by Lemmas 4.4, 4.5 and 4.10, Recall the notations:

$$
\left\|f_{m}\right\|^{2}=\sum_{k=-m}^{m} a_{1 k}^{2}\left(\frac{1}{2 b_{1 k}}+\frac{1}{2 b_{2 k}}\right)^{-1}, \quad\left\|g_{m}\right\|^{2}=\sum_{k=-m}^{m} a_{2 k}^{2}\left(\frac{1}{2 b_{1 k}}+\frac{1}{2 b_{2 k}}\right)^{-1} .
$$

\begin{tabular}{|c|c|c|c|c|c|c|}
\hline table II & $(1)$ & $(2)$ & $(3 \mathrm{a})$ & $(3 \mathrm{~b})$ & $(3 c)$ & $(4)$ \\
\hline$\|f\|^{2}$ & $\infty$ & $<\infty$ & $\infty$ & $\infty$ & $\infty$ & $<\infty$ \\
\hline$\|g\|^{2}$ & $<\infty$ & $\infty$ & $\infty$ & $\infty$ & $\infty$ & $<\infty$ \\
\hline$\frac{\left\|f_{m}\right\|^{2}}{\left\|g_{m}\right\|^{2}}$ & & & $\rightarrow \infty$ & $\rightarrow 0$ & $\begin{array}{l}C_{1} \leq \frac{\left\|f_{m}\right\|^{2}}{\left\|g_{m}\right\|^{2}} \leq \\
C_{2}\end{array}$ & \\
\hline Lemma & $\begin{array}{l}4.4 \\
4.6 \\
\end{array}$ & $\begin{array}{l}4.5 \\
4.7 \\
\end{array}$ & $\begin{array}{l}4.4 \\
4.6 \\
\end{array}$ & \begin{tabular}{|l}
4.5 \\
4.7 \\
\end{tabular} & $\begin{array}{l}4.4,4.5 \\
4.14,4.10 \\
\end{array}$ & \\
\hline & $D_{1 n}, x_{1 n}$ & $D_{2 n}, x_{2 n}$ & $D_{1 n}, x_{1 n}$ & $D_{2 n}, x_{2 n}$ & $D_{1 n}, D_{2 n}$ & \\
\hline
\end{tabular}

All the different cases are presented in the following tables:

Remark 4.6. We show that if $\|g\|^{2}<\infty$ and $S_{12}^{L}(\mu)=\infty$ then $\sum_{n} \frac{b_{1 n}}{b_{2 n}}=\infty$. Indeed, let us suppose that $\sum_{n} \frac{b_{1 n}}{b_{2 n}}<\infty$, then

$$
\|g\|^{2}=\sum_{n \in \mathbb{Z}} \frac{a_{2 n}^{2}}{\frac{1}{2 b_{1 n}}+\frac{1}{2 b_{2 n}}} \sim \sum_{n \in \mathbb{Z}} b_{1 n} a_{2 n}^{2} \sim \sum_{n \in \mathbb{Z}} \frac{b_{1 n}}{2}\left(\frac{1}{2 b_{2 n}}+a_{2 n}^{2}\right)=S_{12}^{L}(\mu)=\infty .
$$

We explain the tables II in details. The first two case (1) and (2) are independent of the case $\mathrm{I}(3)$.

(1) If $\|g\|^{2}<\infty$ and $\|f\|^{2}=\infty$, we have $D_{1 k} \eta \mathfrak{A}$ by Lemma4.4. The condition $\|g\|^{2}<\infty$ implies $\sum_{k \in \mathbb{Z}} \frac{b_{1 k}}{b_{2 k}}=\infty$, by Remark 4.6 therefore, $x_{1 k} \eta \mathfrak{A}$, by Lemma 4.6. Further, $A_{k n}-x_{1 k} D_{1 n}=x_{2 k} D_{2 n}, k, n \in \mathbb{Z}$ and the proof is complete since we are reduced to the case $m=1$. 
(2) If $\|g\|^{2}=\infty$ and $\|f\|^{2}<\infty$, we have $D_{2 k} \eta \mathfrak{A}$ by Lemma 4.5, By remark similar to the Remark 4.6, we conclude that $\sum_{k \in \mathbb{Z}} \frac{b_{2 k}}{b_{1 k}}=\infty$ therefore, $x_{2 k} \eta \mathfrak{A}$ by Lemma 4.7 and $A_{k n}-x_{2 k} D_{2 n}=x_{1 k} D_{1 n}, k, n \in \mathbb{Z}$, case $m=1$.

(3) Consider now the case I(3). Let both series be divergent: $\|g\|^{2}=\infty$ and $\|f\|^{2}=\infty$. We show that in the case (B) (see (4.38)) holds $\|f+s g\|^{2}=\infty$ for all $s \in \mathbb{R}$, by Lemma 4.13 therefore, by Lemma 4.10, we can approximate $D_{1 n}$ and $D_{2 n}$. To be more precise consider three possibilities:

(3a) let $\frac{\left\|f_{m}\right\|^{2}}{\left\|g_{m}\right\|^{2}} \rightarrow \infty$, then $D_{1 k} \eta \mathfrak{A}$. Since $\sum_{n} \frac{b_{1 n}}{b_{2 n}}=\infty$, we have $x_{1 n} \eta \mathfrak{A}$ by Lemma 4.6 and finally, $x_{1 n}, D_{1 n} \eta \mathfrak{A}, n \in \mathbb{Z}$. We are reduced to the case $m=1$.

(3b) Let $\frac{\left\|f_{m}\right\|^{2}}{\left\|g_{m}\right\|^{2}} \rightarrow 0$, then $D_{2 k} \eta \mathfrak{A}$. Since $\sum_{n} \frac{b_{2 n}}{b_{1 n}}=\infty$, we get $x_{2 n} \eta \mathfrak{A}$, by Lemma 4.7 and finally, $x_{2 n}, D_{2 n} \eta \mathfrak{A}, n \in \mathbb{Z}$. We are reduced to the case $m=1$

(3c) The case when $\|f\|^{2}=\|g\|^{2}=\infty$ and $C_{1} \leq \| \frac{\left\|f_{m}\right\|^{2}}{\left\|g_{m}\right\|^{2}} \leq C_{2}$.

(4) The case when $\|f\|^{2}+\|g\|^{2}<\infty$.

To complete the proof of the lemma it remains to consider I(3), i.e., the last case (3) in the table I and the last two cases in the table II, i.e., II(3c) and $\operatorname{II}(4)$, where:

$$
\begin{gathered}
\mathrm{I}(3) \quad \sum_{k \in \mathbb{Z}} \frac{b_{1 k}}{b_{2 k}}=\sum_{k \in \mathbb{Z}} \frac{b_{2 k}}{b_{1 k}}=\infty, \\
\mathrm{II}(\mathrm{c} 3) \sum_{k \in \mathbb{Z}} a_{1 k}^{2}\left(\frac{1}{2 b_{1 k}}+\frac{1}{2 b_{2 k}}\right)^{-1}=\sum_{k \in \mathbb{Z}} a_{2 k}^{2}\left(\frac{1}{2 b_{1 k}}+\frac{1}{2 b_{2 k}}\right)^{-1}=\infty, \\
\mathrm{II}(4) \quad \sum_{k \in \mathbb{Z}}\left(a_{1 k}^{2}+a_{2 k}^{2}\right)\left(\frac{1}{2 b_{1 k}}+\frac{1}{2 b_{2 k}}\right)^{-1}<\infty .
\end{gathered}
$$

Come back to the condition $\mu^{L_{t}} \perp \mu$. By Remark 4.3 we have

$$
\mu^{L_{\tau_{-}(\phi, s)}} \perp \mu, \phi \in[0,2 \pi), s>0 \Leftrightarrow \Sigma_{1}(s)+\Sigma_{2}\left(C_{1}, C_{2}\right)=\infty, s>0,
$$

for $\left(C_{1}, C_{2}\right) \in \mathbb{R}^{2} \backslash\{0\}$. Recall that (see (4.4))

$$
\begin{gathered}
\Sigma_{1}(s)=\sum_{n \in \mathbb{Z}}\left(s^{2} \sqrt{\frac{b_{1 n}}{b_{2 n}}}-s^{-2} \sqrt{\frac{b_{2 n}}{b_{1 n}}}\right)^{2}, \\
\Sigma_{2}\left(C_{1}, C_{2}\right)=\sum_{n \in \mathbb{Z}}\left(C_{1}^{2} b_{1 n}+C_{2}^{2} b_{2 n}\right)\left(C_{1} a_{1 n}+C_{2} a_{2 n}\right)^{2} .
\end{gathered}
$$


The condition $\Sigma_{1}(s)+\Sigma_{2}\left(C_{1}, C_{2}\right)=\infty$ splits into two cases:

$$
\begin{aligned}
& \text { (A) } \Sigma_{1}(s)=\infty, \\
& \text { (B) } \Sigma_{1}(s)<\infty \text { but } \Sigma_{2}\left(C_{1}, C_{2}\right)=\infty .
\end{aligned}
$$

(A) $\& \mathbf{I}(\mathbf{3})$. In this case independently of the conditions II(3c) and II(4) we can approximate $x_{1 n}$ and $x_{2 n}$ by Lemma 4.2 and 4.3 .

(B) $\& \mathbf{I I}(\mathbf{3 c})$ In this case we can approximate $D_{1 n}$ and $D_{2 n}$ by Lemmas 4.4 and 4.5 respectively. More precisely, to use Lemma 4.10 we show that conditions (4.13) are satisfied for two vectors $f$ and $g$ defined by (4.26) (see Lemma 4.14). (B) $\& \mathbf{I I}(4)$ This case (see (4.37)) can not be realized if $\Sigma_{2}\left(C_{1}, C_{2}\right)=\infty$.

Case (A)\&I(3). Using Lemma 4.10 we conclude that

$$
\Gamma\left(f_{m}^{1}, g_{m}^{1}\right)\left(\Gamma\left(g_{m}^{1}\right)\right)^{-1} \rightarrow \infty \quad \text { and } \quad \Gamma\left(f_{m}^{2}, g_{m}^{2}\right)\left(\Gamma\left(g_{m}^{2}\right)\right)^{-1} \rightarrow \infty .
$$

To use Lemma4.10, it is sufficient to show that in the case $(A)$ relations (4.13) hold for $f^{1}, g^{1}$ and $f^{2}, g^{2}$, i.e., for all $s \in \mathbb{R} \backslash\{0\}$ we have (see Lemma 4.13)

$$
\left\|f^{1}\right\|^{2}=\left\|g^{1}\right\|^{2}=\left\|f^{1}+s g^{1}\right\|^{2}=\infty,\left\|f^{2}\right\|^{2}=\left\|g^{2}\right\|^{2}=\left\|f^{2}+s g^{2}\right\|^{2}=\infty .
$$

Consider three possibilities in the case I(3):

(3a) If $\left\|g^{1}\right\|<\infty$ then $\left\|g^{2}\right\|=\infty$ therefore, we have $\left\|f_{m}^{1}\right\| /\left\|g_{m}^{1}\right\| \rightarrow \infty$ so, $x_{1 n} \eta \mathfrak{A}$ by Lemma 4.9 (a). In the case $(A)$ by Lemma 4.13 holds $\left\|f^{2}\right\|^{2}=$ $\left\|g^{2}\right\|^{2}=\left\|f^{2}+s g^{2}\right\|^{2}=\infty$ therefore, $x_{2 n} \eta \mathfrak{A}$ by Lemmat.10.

(3a) If $\left\|g^{2}\right\|<\infty$ then $\left\|g^{1}\right\|=\infty$ therefore, we have $\left\|f_{m}^{2}\right\| /\left\|g_{m}^{2}\right\| \rightarrow \infty$ so, $x_{2 n} \eta \mathfrak{A}$ by Lemma 4.9 (a). In the case $(A)$ by Lemma 4.13 holds $\left\|f^{1}\right\|=$ $\left\|g^{1}\right\|=\left\|f^{1}+s g^{1}\right\|=\infty$ therefore, $x_{1 n} \eta \mathfrak{A}$ by Lemma 4.10.

(3c) If $\left\|g^{1}\right\|=\left\|g^{2}\right\|=\infty$ then by Lemma 4.13 all relations (4.40) hold in the case $(A)$ therefore, $x_{1 n}, x_{2 n} \eta \mathfrak{A}$.

To prove (4.40) we need the following auxiliary lemma.

Lemma 4.12. The following two conditions are equivalent:

$$
\begin{gathered}
\text { (i) } \quad \Sigma_{1}(s)=\sum_{n \in \mathbb{Z}}\left(s^{2} \sqrt{\frac{b_{1 n}}{b_{2 n}}}-s^{-2} \sqrt{\frac{b_{2 n}}{b_{1 n}}}\right)^{2}=\infty, \\
\text { (ii) } \quad \Sigma_{2}(s)=\sum_{n \in \mathbb{Z}}\left(s^{4} \frac{b_{1 n}}{b_{2 n}}-1\right)^{2}+\left(s^{-4} \frac{b_{2 n}}{b_{1 n}}-1\right)^{2}=\infty .
\end{gathered}
$$


Proof. We show that $(i) \Rightarrow(i i)$. Indeed, we have

$$
\left(a^{2}-1\right)^{2}+\left(a^{-2}-1\right)^{2}=\left(a^{2}-1\right)^{2}\left(1+a^{-4}\right)=\left(a-a^{-1}\right)^{2}\left(a^{2}+a^{-2}\right) .
$$

Set $a=s^{2}\left(b_{1 n} / b_{2 n}\right)^{1 / 2}$, then

$$
\Sigma_{2}(s)=\sum_{n \in \mathbb{Z}}\left(s^{2} \sqrt{\frac{b_{1 n}}{b_{2 n}}}-s^{-2} \sqrt{\frac{b_{2 n}}{b_{1 n}}}\right)^{2}\left(s^{4} \frac{b_{1 n}}{b_{2 n}}+s^{-4} \frac{b_{2 n}}{b_{1 n}}\right) \geq 2 \Sigma_{1}(s) .
$$

We prove that $(i i) \Rightarrow(i)$. Denote by $s^{4} \frac{b_{1 n}}{b_{2 n}}=1+a_{n}$, then we have

$$
\begin{gathered}
\Sigma_{1}(s)=\sum_{n \in \mathbb{Z}}\left(\sqrt{1+a_{n}}-\frac{1}{\sqrt{1+a_{n}}}\right)^{2}=\sum_{n \in \mathbb{Z}}\left(\frac{a_{n}}{\sqrt{1+a_{n}}}\right)^{2}=\sum_{n \in \mathbb{Z}} \frac{a_{n}^{2}}{1+a_{n}}, \\
\Sigma_{2}(s)=\sum_{n \in \mathbb{Z}}\left(a_{n}^{2}+\left(\frac{1}{1+a_{n}}-1\right)^{2}\right)=\sum_{n \in \mathbb{Z}}\left(a^{2}+\frac{a_{n}^{2}}{\left(1+a_{n}\right)^{2}}\right)^{\frac{\sqrt{4.9}}{\sim}} \sum_{n \in \mathbb{Z}} a_{n}^{2}+\sum_{n \in \mathbb{Z}} \frac{a_{n}^{2}}{1+a_{n}} .
\end{gathered}
$$

Let $\Sigma_{2}(s)=\infty$. If $\sum_{n \in \mathbb{Z}} \frac{a_{n}^{2}}{1+a_{n}}=\infty$, the proof is complete. Suppose that $\sum_{n \in \mathbb{Z}} a_{n}^{2}=\infty$. We show that in this case $\Sigma_{1}(s)=\infty$. It is sufficient to prove that

$$
\sum_{n \in \mathbb{N}} a_{n}^{2}=\infty \text { implies } \sum_{n \in \mathbb{N}} a_{n}^{2}\left(1+a_{n}\right)^{-1}=\infty .
$$

Consider three cases:

(a) If $0<\varepsilon \leq 1+a_{n} \leq C<\infty$ for all $n \in \mathbb{N}$, then

$$
C^{-1} \sum_{n \in \mathbb{N}} a_{n}^{2} \leq \sum_{n \in \mathbb{N}} a_{n}^{2}\left(1+a_{n}\right)^{-1} \leq \varepsilon^{-1} \sum_{n \in \mathbb{N}} a_{n}^{2} .
$$

(b) If $\lim _{k \rightarrow \infty}\left(1+a_{n_{k}}\right)=0$, then

$$
\lim _{k \rightarrow \infty} a_{n_{k}}^{2}\left(1+a_{n_{k}}\right)^{-1}=\infty \quad \text { and } \quad \sum_{n \in \mathbb{N}} a_{n}^{2}\left(1+a_{n}\right)^{-1}=\infty .
$$

(c) If $\lim _{k \rightarrow \infty}\left(1+a_{n_{k}}\right)=+\infty$, then

$$
\sum_{n \in \mathbb{N}} a_{n}^{2}\left(1+a_{n}\right)^{-1}>\sum_{k \in \mathbb{N}} a_{n_{k}}\left(a_{n_{k}}^{-1}+1\right)^{-1} \sim \sum_{k \in \mathbb{N}} a_{n_{k}}=\infty .
$$


Lemma 4.13. If $\Sigma_{1}(s)=\infty$ for any $s>0$, then

$$
\left\|f^{1}-C g^{1}\right\|^{2}=\infty \quad \text { and } \quad\left\|f^{2}-C g^{2}\right\|^{2}=\infty, \text { for any } C>0 .
$$

Proof. Set as before $c_{n}=\frac{b_{2 n}}{b_{1 n}}, n \in \mathbb{Z}$. Suppose that $\Sigma_{1}(s)=\infty$, then

$$
\infty=\Sigma_{1}(s)=\sum_{n \in \mathbb{Z}}\left(\frac{s^{2}}{\sqrt{c_{n}}}-\frac{\sqrt{c_{n}}}{s^{2}}\right)^{2}=\sum_{n \in \mathbb{Z}} \frac{a_{n}^{2}}{1+a_{n}},
$$

where $s^{4} c_{n}^{-1}=1+a_{n}$ or $c_{n}=\frac{s^{4}}{1+a_{n}}$. We show that

$$
\left\|s^{4} f^{1}-g^{1}\right\|^{2}=\infty \quad \text { and } \quad\left\|s^{-4} f^{2}-C g^{2}\right\|^{2}=\infty
$$

Indeed, using (4.30) and (4.31) we get

$$
\begin{gathered}
\left\|s^{4} f^{1}-g^{1}\right\|^{2}=\sum_{k \in \mathbb{Z}} \frac{\left(s^{4}-c_{k}\right)^{2}}{1+2 c_{k}}=\sum_{k \in \mathbb{Z}}\left(\frac{s^{4}}{c_{k}}-1\right)\left(\frac{1}{c_{k}^{2}}+\frac{2}{c_{k}}\right)^{-1}= \\
\sum_{k \in \mathbb{Z}} \frac{a_{k}^{2}}{\left(\frac{1+a_{k}}{s^{4}}\right)^{2}+2 \frac{1+a_{k}}{s^{4}}} \sim \sum_{k \in \mathbb{Z}} \frac{a_{k}^{2}}{\left(1+a_{k}\right)^{2}+2\left(1+a_{k}\right)}= \\
\sum_{k \in \mathbb{Z}} \frac{a_{k}^{2}}{3+4 a_{k}+a_{k}^{2}} \sim \sum_{k \in \mathbb{Z}} \frac{a_{k}^{2}}{1+a_{k}}=\infty
\end{gathered}
$$

and

$$
\begin{gathered}
\left\|s^{-4} f^{2}-g^{2}\right\|^{2}=\sum_{k \in \mathbb{Z}} \frac{\left(s^{-4} c_{k}^{2}-1\right)^{2}}{c_{k}^{2}+2 c_{k}}= \\
\sum_{k \in \mathbb{Z}}\left(\frac{1}{1+a_{k}}-1\right)^{2}\left(\left(\frac{s^{4}}{1+a_{k}}\right)^{2}+2 \frac{s^{4}}{1+a_{k}}\right)^{-1}= \\
\sum_{k \in \mathbb{Z}} \frac{a_{k}^{2}}{s^{8}+2 s^{4}\left(1+a_{k}\right)} \sim \sum_{k \in \mathbb{Z}} \frac{a_{k}^{2}}{1+a_{k}}=\infty .
\end{gathered}
$$

So, in the case $(\mathrm{A}) \& \mathrm{I}(3)$ we can approximate $x_{1 n}$ and $x_{2 n}$.

Case $(\mathrm{B}) \& \mathrm{II}(3 \mathrm{c})$. 
Lemma 4.14. When $\Sigma_{1}(s)<\infty$ and $\Sigma_{2}\left(C_{1}, C_{2}\right)=\infty$, we get

$\sigma\left(C_{1}, C_{2}\right):=\left\|C_{1} f+C_{2} g\right\|^{2}=\sum_{n \in \mathbb{Z}} \frac{\left(C_{1} a_{1 n}+C_{2} a_{2 n}\right)^{2}}{\frac{1}{2 b_{1 n}}+\frac{1}{2 b_{2 n}}}=\infty, \quad\left(C_{1}, C_{2}\right) \in \mathbb{R}^{2} \backslash\{0\}$,

where $f$ and $g$ are defined by 4.28)

$$
f=\left(a_{1 k}\left(\frac{1}{2 b_{1 k}}+\frac{1}{2 b_{2 k}}\right)^{-1 / 2}\right)_{k \in \mathbb{Z}}, \quad g=\left(a_{2 k}\left(\frac{1}{2 b_{1 k}}+\frac{1}{2 b_{2 k}}\right)^{-1 / 2}\right)_{k \in \mathbb{Z}} .
$$

ProOF. Let $\Sigma_{1}(s)=\sum_{n \in \mathbb{Z}} \frac{a_{n}^{2}}{1+a_{n}}<\infty$, where $s^{4} \frac{b_{1 n}}{b_{2 n}}=1+a_{n}$ or $s^{4} b_{1 n}=$ $\left(1+a_{n}\right) b_{2 n}$. We see that $\lim _{n} \frac{a_{n}^{2}}{1+a_{n}}=0$ hence, $\lim _{n} a_{n}=\lim _{n}\left(s^{4} \frac{b_{1 n}}{b_{2 n}}-1\right)=0$. We have

$$
\begin{gathered}
\sigma\left(C_{1}, C_{2}\right)=\sum_{n \in \mathbb{Z}} \frac{b_{1 n}\left(C_{1} a_{1 n}+C_{2} a_{2 n}\right)^{2}}{\frac{1}{2}+\frac{b_{1 n}}{2 b_{2 n}}}=\sum_{n \in \mathbb{Z}} \frac{b_{1 n}\left(C_{1} a_{1 n}+C_{2} a_{2 n}\right)^{2}}{\frac{1}{2}+\frac{1}{2} \frac{1+a_{n}}{s^{4}}} \\
\sim \sum_{n \in \mathbb{Z}} C_{1}^{2} b_{1 n}\left(C_{1} a_{1 n}+C_{2} a_{2 n}\right)^{2}, \\
\sigma\left(C_{1}, C_{2}\right)=\sum_{n \in \mathbb{Z}} \frac{b_{2 n}\left(C_{1} a_{1 n}+C_{2} a_{2 n}\right)^{2}}{\frac{b_{2 n}}{2 b_{1 n}}+\frac{1}{2}}=\sum_{n \in \mathbb{Z}} \frac{b_{2 n}\left(C_{1} a_{1 n}+C_{2} a_{2 n}\right)^{2}}{\frac{1}{2} \frac{s^{4}}{1+a_{n}}+\frac{1}{2}} \\
\sim \sum_{n \in \mathbb{Z}} C_{2}^{2} b_{2 n}\left(C_{1} a_{1 n}+C_{2} a_{2 n}\right)^{2},
\end{gathered}
$$

hence, $\sigma\left(C_{1}, C_{2}\right) \sim \sum_{n \in \mathbb{Z}}\left(C_{1}^{2} b_{1 n}+C_{2}^{2} b_{2 n}\right)\left(C_{1} a_{1 n}+C_{2} a_{2 n}\right)^{2}=\Sigma_{2}\left(C_{1}, C_{2}\right)$.

Finally, we can approximate $D_{1 n}$ and $D_{2 n}$ in the case $(\mathrm{B}) \& \mathrm{II}(3 \mathrm{c})$.

Case (B)\&II(4). The last case (B)\&II(4) (see (4.37)) can not be realized if $\Sigma_{2}\left(C_{1}, C_{2}\right)=\infty$. Indeed, in this case by Lemma $4.14 \sigma\left(C_{1}, C_{2}\right) \sim$ $\Sigma_{2}\left(C_{1}, C_{2}\right)=\infty$. This contradicts (4.37):

$$
\sum_{k \in \mathbb{Z}}\left(a_{1 k}^{2}+a_{2 k}^{2}\right)\left(\frac{1}{2 b_{1 k}}+\frac{1}{2 b_{2 k}}\right)^{-1}<\infty .
$$

This completes the proof of Lemma 4.11 for $m=2$. 
The proof of the irreducibility for $m=2$ follows from Remark 2.2. Depending on the measure, we can approximate four different families of commuting operators $B^{\alpha}=\left(B_{1 n}^{\alpha}, B_{2 n}^{\alpha}\right)_{n \in \mathbb{Z}}$ for $\alpha \in\{0,1\}^{2}$ :

$B^{(0,0)}=\left(x_{1 n}, x_{2 n}\right)_{n}, B^{(0,1)}=\left(x_{1 n}, D_{2 n}\right)_{n}, B^{(1,0)}=\left(D_{1 n}, x_{2 n}\right)_{n}, B^{(0,0)}=\left(D_{1 n}, D_{2 n}\right)_{n}$.

The von Neumann algebra $L_{\alpha}^{\infty}\left(X_{2}, \mu^{2}\right)$ consists of all essentially bounded functions $f\left(B^{\alpha}\right)$ in the commuting family of operators $B^{\alpha}$ (see, e.g., [4]) as, in particular, $L_{(0,0)}^{\infty}\left(X_{2}, \mu^{2}\right)=L^{\infty}\left(X_{2}, \mu^{2}\right)$. Since the von Neumann algebras $L_{\alpha}^{\infty}\left(X_{2}, \mu^{2}\right)$ are maximal abelian, the commutant $\left(\mathfrak{A}^{2}\right)^{\prime}$ of the von Neumann algebra $\mathfrak{A}^{2}$ generated by the representation is contained in $L_{\alpha}^{\infty}\left(X_{2}, \mu^{2}\right)$. Hence, the bounded operator $A \in\left(\mathfrak{A}^{2}\right)^{\prime}$ will be some function $A=a\left(B^{\alpha}\right) \in$ $L_{\alpha}^{\infty}\left(X_{2}, \mu^{2}\right)$. The commutation relation $\left[A, T_{t}^{R, \mu, 2}\right]=0$ gives us the following relations: $a\left(\left(B^{\alpha}\right)^{R_{t}}\right)=a\left(B^{\alpha}\right)$ for all $t \in \mathrm{GL}_{0}(2 \infty, \mathbb{R})$. Set $B_{r}^{\alpha}=$ $\left(B_{r n}^{\alpha}\right)_{n}, x_{r}=\left(x_{r n}\right)_{n}, D_{r}=\left(x_{r n}\right)_{n}, r=1,2, n \in \mathbb{Z}$ and set as before, $E_{k n}(t):=I+t E_{k n}, t \in \mathbb{R}, k, n \in \mathbb{Z}, k \neq n$. Then the action $\left(B^{\alpha}\right)^{R_{s}}$ is defined as follows:

$$
\begin{array}{r}
\left(B_{1}^{\alpha}, B_{2}^{\alpha}\right)^{R_{t}}=\left(\left(B_{1}^{\alpha}\right)^{R_{t}},\left(B_{2}^{\alpha}\right)^{R_{t}}\right), \quad\left(x_{r}\right)^{R_{t}}=x_{r} t, \quad\left(D_{r}\right)^{R_{t}}=D_{r} t^{*}, \\
a\left(\ldots, x_{r k}, \ldots, x_{r n}, \ldots\right)^{R_{E_{k n}(t)}}=a\left(\ldots, x_{r k}, \ldots, x_{r n}+t x_{r k}, \ldots\right), \\
a\left(\ldots, D_{r k}, \ldots, D_{r n}, \ldots\right)^{R_{E_{k n}(t)}}=a\left(\ldots, D_{r k}+t D_{r n}, \ldots, D_{r n}, \ldots\right), \quad t \in \mathbb{R} .
\end{array}
$$

In all the cases, by ergodicity of the measure $\mu^{2}$, we conclude that $a$ is constant.

\subsection{The proof of Lemmas 2.2, 4.1]}

Lemma 2.2 follows from Lemmas 4.15 4.18.

Lemma 4.15. For $t \in \mathrm{GL}(m, \mathbb{R}) \backslash\{e\}$ we have $\left(\mu_{(b, a)}^{m}\right)^{L_{t}} \perp \mu_{(b, a)}^{m}$ if and only if

$$
\left(\mu_{(b, 0)}^{m}\right)^{L_{t}} \perp \mu_{(b, 0)}^{m} \quad \text { or } \quad \mu_{\left(b, L_{t} a\right)}^{m} \perp \mu_{(b, a)}^{m} .
$$

Let us define the following measures on the spaces $\mathbb{R}^{m}$ and $X_{m}$ :

$$
\mu_{m}^{\left(B_{n}, 0\right)}=\otimes_{k=1}^{m} \mu_{\left(b_{k n}, 0\right)}, \quad \mu_{m}^{\left(B_{n}, a_{n}\right)}=\otimes_{k=1}^{m} \mu_{\left(b_{k n}, a_{k n}\right)},
$$

where $a_{n}=\left(a_{1 n}, \ldots, a_{m n}\right) \in \mathbb{R}^{m}$ and $B_{n}=\operatorname{diag}\left(b_{1 n}, \ldots, b_{m n}\right) \in \operatorname{Mat}(m, \mathbb{R})$. Since

$$
\mu_{(b, a)}^{m}=\otimes_{n \in \mathbb{Z}} \mu_{m}^{\left(B_{n}, a_{n}\right)}, \quad \mu_{(b, 0)}^{m}=\otimes_{n \in \mathbb{Z}} \mu_{m}^{\left(B_{n}, 0\right)}
$$




$$
\left(\mu_{(b, a)}^{m}\right)^{L_{t}}=\otimes_{n \in \mathbb{Z}}\left(\mu_{m}^{\left(B_{n}, a_{n}\right)}\right)^{L_{t}}, \quad\left(\mu_{(b, 0)}^{m}\right)^{L_{t}}=\otimes_{n \in \mathbb{Z}}\left(\mu_{m}^{\left(B_{n}, 0\right)}\right)^{L_{t}},
$$

and

$$
\mu_{\left(b, L_{t} a\right)}^{m}=\otimes_{n \in \mathbb{Z}} \mu_{m}^{\left(B_{n}, L_{t} a_{n}\right)},
$$

by Kakutani criterion [11], we have two lemmas:

Lemma 4.16. For measures $\mu_{(b, 0)}^{m}, m \in \mathbb{N}$ and $t \in \mathrm{GL}(m, \mathbb{R}) \backslash\{e\}$ we obtain

$$
\left(\mu_{(b, 0)}^{m}\right)^{L_{t}} \perp \mu_{(b, 0)}^{m} \Leftrightarrow \prod_{n \in \mathbb{Z}} H\left(\left(\mu_{m}^{\left(B_{n}, 0\right)}\right)^{L_{t}}, \mu_{m}^{\left(B_{n}, 0\right)}\right)=0 .
$$

Lemma 4.17. For measures $\mu_{(b, 0)}^{m}, m \in \mathbb{N}$ and $t \in \mathrm{GL}(m, \mathbb{R}) \backslash\{e\}$ we get

$$
\mu_{\left(b, L_{t} a\right)}^{m} \perp \mu_{(b, a)}^{m} \Leftrightarrow \prod_{n \in \mathbb{Z}} H\left(\mu_{m}^{\left(B_{n}, L_{t} a_{n}\right)}, \mu_{m}^{\left(B_{n}, a_{n}\right)}\right)=0 .
$$

To prove Lemma 2.2 it is sufficient to show, due to Lemma 4.15, that

$$
H\left(\left(\mu_{m}^{\left(B_{n}, 0\right)}\right)^{L_{t}}, \mu_{m}^{\left(B_{n}, 0\right)}\right)=\left(\frac{1}{2^{m}|\operatorname{det} t|} \operatorname{det}\left(I+X_{n}^{*}(t) X_{n}(t)\right)\right)^{-1 / 2},
$$

to prove the equivalence

$$
\prod_{n \in \mathbb{Z}} H\left(\mu_{m}^{\left(B_{n}, L_{t} a_{n}\right)}, \mu_{m}^{\left(B_{n}, a_{n}\right)}\right)=0 \Leftrightarrow \sum_{n \in \mathbb{Z}} \sum_{r=1}^{m} b_{r n}\left(\sum_{s=1}^{m}\left(t_{r s}-\delta_{r s}\right) a_{s n}\right)^{2}=\infty,
$$

and to use the following lemma:

Lemma 4.18. For $X \in \operatorname{Mat}(m, \mathbb{R})$ we have

$$
\operatorname{det}\left(I+X^{*} X\right)=1+\sum_{r=1}^{m} \sum_{1 \leq i_{1}<i_{2}<\ldots<i_{r} \leq m ; 1 \leq j_{1}<j_{2}<\ldots<j_{r} \leq m}\left(M_{j_{1} j_{2} \ldots j_{r}}^{i_{1} i_{2} \ldots i_{r}}(X)\right)^{2} .
$$

The proof of equality (4.45) is based on the exact formula of the Hellinger integral (see 23] for definition) for two Gaussian measures $\mu=\mu_{m}^{\left(B_{n}, 0\right)}$ and $\nu=\mu_{m}^{\left(C_{n}, 0\right)}$ in the space $\mathbb{R}^{m}$ (see [23])

$$
H(\mu, \nu)=\int_{X} \sqrt{\frac{d \mu}{d \rho} \frac{d \nu}{d \rho}} d \rho=\left(\frac{\operatorname{det} B_{n} \operatorname{det} C_{n}}{\operatorname{det}^{2} \frac{B_{n}+C_{n}}{2}}\right)^{1 / 4} .
$$


The latter formula is based on the following formula for a positive definite operator $C$ in the space $\mathbb{R}^{m}$ :

$$
\frac{1}{\sqrt{\pi^{m}}} \int_{\mathbb{R}^{m}} \exp (-(C x, x)) d x=\frac{1}{\sqrt{\operatorname{det} C}} .
$$

Let, as before, $t=\left(t_{r s}\right)_{r, s=1}^{m} \in \mathrm{GL}(m, \mathbb{R}), B_{n}=\operatorname{diag}\left(b_{1 n}, b_{2 n}, \ldots, b_{m n}\right), X_{n}(t)=$ $B_{n}^{1 / 2} t B_{n}^{-1 / 2} \in \operatorname{Mat}(m, \mathbb{R})$. Let $M_{j_{1} j_{2} \ldots j_{r}}^{i_{1} i_{2} \ldots i_{r}}(t)$ be the minors of the matrix $t$ with $i_{1}, i_{2}, \ldots, i_{r}$ rows and $j_{1}, j_{2}, \ldots, j_{r}$ columns.

Let us denote by $\mu^{(B, a)}=\mu_{(C, a)}$ the Gaussian measure with the covariance operator $C=(2 B)^{-1}$ on the space $\mathbb{R}^{m}$ defined by the formula: $\frac{d \mu^{B}(x)}{d x}=$

$$
\left.\sqrt{\frac{\operatorname{det} B}{\pi^{m}}} \exp (-(B x, x))=\frac{1}{\sqrt{(2 \pi)^{m} \operatorname{det} C}} \exp \left(-\frac{1}{2}\left(C^{-1} x, x\right)\right)\right)=\frac{d \mu_{C}(x)}{d x} .
$$

Recall that by definition $\mu^{f}(\Delta)=\mu\left(f^{-1}(\Delta)\right)$. Since $L_{t} x=t x$, we get $\mu^{L_{t}^{-1}}(x)=\mu(t x)$ therefore,

$$
\left(\mu_{m}^{\left(B_{n}, a\right)}\right)^{L_{t}^{-1}}(x)=\mu_{m}^{\left(B_{n}(t), t^{-1} a\right)} \quad \text { where } \quad B_{n}(t)=t^{*} B_{n} t .
$$

Indeed,

$$
\begin{gathered}
d\left(\mu_{m}^{\left(B_{n}, a\right)}\right)^{L_{t}^{-1}}(x)=\sqrt{\frac{\operatorname{det} B_{n}}{\pi^{m}}} \exp \left(-\left(B_{n} t\left(x-t^{-1} a\right), t\left(x-t^{-1} a\right)\right)\right) d t x= \\
\sqrt{\frac{|\operatorname{det} t|^{2} \operatorname{det} B_{n}}{\pi^{m}}} \exp \left(-\left(t^{*} B_{n} t\left(x-t^{-1} a\right),\left(x-t^{-1} a\right)\right)\right) d x=d \mu_{m}^{\left(B_{n}(t), t^{-1} a\right)}(x),
\end{gathered}
$$

where $B_{n}(t)=t^{*} B_{n} t, B_{n}=\operatorname{diag}\left(b_{1 n}, b_{2 n}, \ldots, b_{m n}\right)$, $\operatorname{det} B_{n}(t)=|\operatorname{det} t|^{2} \operatorname{det} B_{n}$.

Using (4.48), (4.50) and (4.51) we obtain

$$
\begin{gathered}
H\left(\left(\mu_{m}^{\left(B_{n}, 0\right)}\right)^{L_{t}}, \mu_{m}^{\left(B_{n}, 0\right)}\right)=H\left(\mu_{m}^{\left(B_{n}(t), 0\right)}, \mu_{m}^{\left(B_{n}, 0\right)}\right)=\left(\frac{\operatorname{det} B_{n}(t) \operatorname{det} B_{n}}{\pi^{m} \pi^{m}}\right)^{1 / 4} \times \\
\int_{\mathbb{R}^{m}} \exp \left(-\left(\frac{B_{n}(t)+B_{n}}{2} x, x\right)\right) d x=\left(\frac{\operatorname{det} B_{n}(t) \operatorname{det} B_{n}}{\operatorname{det}^{2} \frac{B_{n}(t)+B_{n}}{2}}\right)^{1 / 4}=\left(\frac{\operatorname{det} C_{n}(t)}{|\operatorname{det} t| \operatorname{det} B_{n}}\right)^{-1 / 2},
\end{gathered}
$$

where $C_{n}(t)=\frac{B_{n}(t)+B_{n}}{2}=\frac{t^{*} B_{n} t+B_{n}}{2}$. Now we show that

$$
\frac{\operatorname{det} C_{n}(t)}{|\operatorname{det} t| \operatorname{det} B_{n}}=\frac{1}{2^{m}|\operatorname{det} t|} \operatorname{det}\left(I+X_{n}^{*}(t) X_{n}(t)\right)
$$


where $X_{n}(t)=B_{n}^{1 / 2} t B_{n}^{-1 / 2}$. The latter equation is equivalent to

$$
\frac{\operatorname{det}\left(t^{*} B_{n} t+B_{n}\right)}{\operatorname{det} B_{n}}=\operatorname{det}\left(I+X_{n}^{*}(t) X_{n}(t)\right) \text {. }
$$

To complete the proof of (4.45) it is sufficient to see that

$$
I+X_{n}^{*}(t) X_{n}(t)=I+B_{n}^{-1 / 2} t^{*} B_{n}^{1 / 2} B_{n}^{1 / 2} t B_{n}^{-1 / 2}=B_{n}^{-1 / 2}\left(B_{n}+t^{*} B_{n} t\right) B_{n}^{-1 / 2} .
$$

The proof of relation (4.46) is based on the following theorem that one can find, e.g., in [29, Ch. III, §16, Theorem 2].

Theorem 4.19. Two Gaussian measures $\mu_{B, a}$ and $\mu_{B, b}$ are equivalent if and only of $B^{-1 / 2}(a-b) \in H$.

Indeed, we have

$$
\left\|C^{-1 / 2}(t a-a)\right\|_{H}^{2}=\sum_{n \in \mathbb{Z}}\left\|C_{n}^{-1 / 2}(t-I) a_{n}\right\|_{H_{n}}^{2}=2 \sum_{n \in \mathbb{Z}} \sum_{r=1}^{m} \frac{b_{k n}}{d_{k n}}\left(\sum_{s=1}^{m}\left(t_{r s}-\delta_{r s}\right) a_{s n}\right)^{2} d_{k n} .
$$

To explain the latter equality let us describe $H$ and $C$. To find an operator $C$ we present the measure $\mu_{(b, a)}^{m}$ in the canonical form $\mu_{C, a}$ defined by its Fourier transform:

$$
\int_{H} \exp i(y, x) d \mu_{C, a}(x)=\exp \left(i(a, y)-\frac{1}{2}(C y, y)\right), y \in H,
$$

where $C$ is a positive nuclear operator (called the covariance operator) on the Hilbert space $H$, and $a \in H$ is the mathematical expectation or mean.

Recall the Kolmogorov zero-one law. Let us consider in the space $\mathbb{R}^{\infty}=$ $\mathbb{R} \times \mathbb{R} \times \cdots$ the infinite tensor product $\mu_{b}=\otimes_{n \in \mathbb{N}} \mu_{b_{k}}$ of one-dimensional Gaussian measures $\mu_{b_{k}}$ on $\mathbb{R}$ defined as follows:

$$
d \mu_{b}(x)=\sqrt{b / \pi} \exp \left(-b x^{2}\right) d x .
$$

Consider a Hilbert space $l_{2}(a)$ defined by

$$
l_{2}(a)=\left\{x \in \mathbb{R}^{\infty}:\|x\|_{l_{2}(a)}^{2}=\sum_{k \in \mathbb{N}} x_{k}^{2} a_{k}<\infty\right\}
$$

where $a=\left(a_{k}\right)_{k \in \mathbb{N}}$ is an infinite sequence of positive numbers. 
Theorem 4.20 (Kolmogorov's zero-one law, [28]). We have

$$
\mu_{b}\left(l_{2}(a)\right)= \begin{cases}0, & \text { if } \sum_{k \in \mathbb{N}} \frac{a_{k}}{b_{k}}=\infty \\ 1, & \text { if } \sum_{k \in \mathbb{N}} \frac{a_{k}}{b_{k}}<\infty\end{cases}
$$

Define the Hilbert space $H \subset X_{m}$ as follows:

$$
H=l_{2}\left(\mathbb{R}^{m}, d\right)=\left\{x=\left(x_{k n}\right)_{k, n} \in X_{m} \mid\|x\|_{H}^{2}:=\sum_{1 \leq k \leq m, n \in \mathbb{N}} x_{k n}^{2} d_{k n}<\infty\right\},
$$

where a sequence $d=\left(d_{k n}\right)_{1 \leq k \leq m, n \in \mathbb{Z}}$ of positive numbers is chosen such that $\sum_{1 \leq k \leq m, n \in \mathbb{N}} \frac{d_{k n}}{b_{k n}}<\infty$. Then by the Kolmogorov zero-one law, $\mu_{(b, a)}^{m}(H)=1$. We show that $C=\operatorname{diag}\left(c_{k n}\right)$, where $c_{k n}=\frac{d_{k n}}{2 b_{k n}}$. Indeed, we get

$$
\sum_{1 \leq k \leq m, n \in \mathbb{N}} b_{k n} x_{k n}^{2}=\frac{1}{2} \sum_{1 \leq k \leq m, n \in \mathbb{N}} \frac{2 b_{k n}}{d_{k n}} x_{k n}^{2} d_{k n}=\frac{1}{2}\left(C^{-1} x, x\right)_{H} .
$$

Proof of Lemma 4.18. Let as recall the definition of the Gram determinant and the Gram matrix (see [7], Chap IX, §5). For vectors $x_{1}, x_{2}, \ldots, x_{m}$ in some Hilbert space $H$ the Gram matrix $\gamma\left(x_{1}, x_{2}, \ldots, x_{m}\right)$ is defined by the formula

$$
\gamma\left(x_{1}, x_{2}, \ldots, x_{m}\right)=\left(\left(x_{k}, x_{n}\right)_{k, n=1}^{m}\right) .
$$

The determinant of this matrix is called the Gram determinant for the vectors $x_{1}, x_{2}, \ldots, x_{m}$ and is denoted by $\Gamma\left(x_{1}, x_{2}, \ldots, x_{m}\right)$. Thus,

$$
\Gamma\left(x_{1}, x_{2}, \ldots, x_{m}\right):=\operatorname{det} \gamma\left(x_{1}, x_{2}, \ldots, x_{m}\right) .
$$

Let

$$
X=\left(\begin{array}{cccc}
x_{11} & x_{12} & \ldots & x_{1 m} \\
x_{21} & x_{22} & \ldots & x_{2 m} \\
\ldots & \ldots & \ldots & \ldots \\
x_{m 1} & x_{m 2} & \ldots & x_{m m}
\end{array}\right)
$$

Set $x_{k}=\left(x_{1 k}, x_{2 k}, \ldots, x_{m k}\right) \in \mathbb{R}^{m}, 1 \leq k \leq m$, then, obviously, we get

$$
X^{*} X=\left(\begin{array}{cccc}
\left(x_{1}, x_{1}\right) & \left(x_{1}, x_{2}\right) & \ldots & \left(x_{1}, x_{m}\right) \\
\left(x_{2}, x_{1}\right) & \left(x_{2}, x_{2}\right) & \ldots & \left(x_{2}, x_{m}\right) \\
\ldots & \ldots & \ldots & \ldots \\
\left(x_{m}, x_{1}\right) & \left(x_{m}, x_{2}\right) & \ldots & \left(x_{m}, x_{m}\right)
\end{array}\right)=\gamma\left(x_{1}, x_{2}, \ldots, x_{m}\right)
$$


We would like to find an exact expression for $\operatorname{det}\left(I+\gamma\left(x_{1}, x_{2}, \ldots, x_{m}\right)\right)$. It is convenient to consider the following function:

$$
F_{m, X}^{\lambda}=F_{m ; x_{1}, x_{2}, \ldots, x_{m}}^{\lambda_{1}, \lambda_{2}, \ldots, \lambda_{m}}=\operatorname{det}\left(\sum_{k=1}^{m} \lambda_{k} E_{k k}+\gamma\left(x_{1}, x_{2}, \ldots, x_{m}\right)\right), \quad \lambda \in \mathbb{C}^{m}
$$

It is easy to see that for $m=2$ we have

$$
\begin{gathered}
F_{2 ; x_{1}, x_{2}}^{\lambda_{1}, \lambda_{2}}=\operatorname{det}\left(\begin{array}{cc}
\lambda_{1}+\left(x_{1}, x_{1}\right) & \left(x_{1}, x_{2}\right) \\
\left(x_{2}, x_{1}\right) & \lambda_{2}+\left(x_{2}, x_{2}\right)
\end{array}\right)= \\
\lambda_{1} \lambda_{2}+\lambda_{1} \Gamma\left(x_{2}\right)+\lambda_{2} \Gamma\left(x_{1}\right)+\Gamma\left(x_{1}, x_{2}\right)= \\
\lambda_{1} \lambda_{2}\left(1+\lambda_{1}^{-1} \Gamma\left(x_{1}\right)+\lambda_{2}^{-1} \Gamma\left(x_{2}\right)+\left(\lambda_{1} \lambda_{2}\right)^{-1} \Gamma\left(x_{1}, x_{2}\right)\right) .
\end{gathered}
$$

The general formula is

$$
\begin{aligned}
& F_{m ; x_{1}, x_{2}, \ldots, x_{m}}^{\lambda_{1}, \lambda_{2}, \ldots, \lambda_{m}}=\operatorname{det}\left(\sum_{k=1}^{m} \lambda_{k} E_{k k}+\gamma\left(x_{1}, x_{2}, \ldots, x_{m}\right)\right)= \\
& \prod_{k=1}^{m} \lambda_{k}\left(1+\sum_{r=1}^{m} \sum_{1 \leq i_{1}<i_{2}<\ldots<i_{r} \leq m}\left(\lambda_{i_{1}} \lambda_{i_{2}} \ldots \lambda_{i_{r}}\right)^{-1} \Gamma\left(x_{i_{1}}, x_{i_{2}}, \ldots, x_{i_{r}}\right)\right)= \\
& \prod_{k=1}^{m} \lambda_{k}\left(1+\sum_{r=1}^{m} \sum_{1 \leq i_{1}<i_{2}<\ldots<i_{r} \leq m ; 1 \leq j_{1}<j_{2}<\ldots<j_{r} \leq m}\left(\lambda_{i_{1}} \lambda_{i_{2}} \ldots \lambda_{i_{r}}\right)^{-1}\left(M_{j_{1} j_{2} \ldots j_{r}}^{i_{1} i_{2} \ldots i_{r}}(X)\right)^{2}\right) .
\end{aligned}
$$

We have used the following formula (see [7], Chap IX, $\S 5$ formula (25)):

$$
\Gamma\left(x_{i_{1}}, x_{i_{2}}, \ldots, x_{i_{r}}\right)=\sum_{1 \leq j_{1}<j_{2}<\ldots<j_{r} \leq m}\left(M_{j_{1} j_{2} \ldots j_{r}}^{i_{1} i_{2} \ldots i_{r}}(X)\right)^{2}
$$

Finally, using (4.56) for $\left(\lambda_{1}, \lambda_{2}, \ldots, \lambda_{m}\right)=(1,1, \ldots, 1)$ we get (4.47).

We study the case $m=2$ more carefully.

Lemma 4.21. For $t \in G L(2, \mathbb{R})$ we have, if $\operatorname{det} t>0$,

$$
\begin{gathered}
\left(\mu_{(b, 0)}^{2}\right)^{L_{t}} \perp \mu_{(b, 0)}^{2} \Leftrightarrow \\
\sum_{n \in \mathbb{Z}}\left[(1-|\operatorname{det} t|)^{2}+\left(t_{11}-t_{22}\right)^{2}+\left(t_{12} \sqrt{\frac{b_{1 n}}{b_{2 n}}}+t_{21} \sqrt{\frac{b_{2 n}}{b_{1 n}}}\right)^{2}\right]=\infty .
\end{gathered}
$$


If $\operatorname{det} t<0$ we have

$$
\begin{gathered}
\left(\mu_{(b, 0)}^{2}\right)^{L_{t}} \perp \mu_{(b, 0)}^{2} \Leftrightarrow \\
\sum_{n \in \mathbb{Z}}\left[(1-|\operatorname{det} t|)^{2}+\left(t_{11}+t_{22}\right)^{2}+\left(t_{12} \sqrt{\frac{b_{1 n}}{b_{2 n}}}-t_{21} \sqrt{\frac{b_{2 n}}{b_{1 n}}}\right)^{2}\right]=\infty .
\end{gathered}
$$

Proof. Using (4.45) set

$H_{m, n}(t)=H\left(\left(\mu_{m}^{\left(B_{n}, 0\right)}\right)^{L_{t}^{-1}}, \mu_{m}^{\left(B_{n}, 0\right)}\right)=\left(\frac{1}{2^{m}|\operatorname{det} t|} \operatorname{det}\left(I+X_{n}^{*}(t) X_{n}(t)\right)\right)^{-1 / 2}$.

For $m=2$ using (2.5) we get $X(t)=B^{1 / 2} t B^{-1 / 2}$ hence,

$X(t)=\left(\begin{array}{cc}b_{1 n} & 0 \\ 0 & b_{2 n}\end{array}\right)^{1 / 2}\left(\begin{array}{cc}t_{11} & t_{12} \\ t_{21} & t_{22}\end{array}\right)\left(\begin{array}{cc}b_{1 n} & 0 \\ 0 & b_{2 n}\end{array}\right)^{-1 / 2}=\left(\begin{array}{cc}t_{11} & \sqrt{\frac{b_{1 n}}{b_{2 n}}} t_{12} \\ \sqrt{\frac{b_{2 n}}{b_{1 n}}} t_{21} & t_{22}\end{array}\right)$.

Therefore, using (4.55) we get

$$
H_{2, n}^{-2}(t)=\frac{1}{2^{2}|\operatorname{det} t|}\left(1+|\operatorname{det} t|^{2}+t_{11}^{2}+t_{22}^{2}+\frac{b_{1 n}}{b_{2 n}} t_{12}^{2}+\frac{b_{2 n}}{b_{1 n}} t_{21}^{2}\right) .
$$

Using Lemma4.16 it is sufficient to calculate $H_{2, n}^{-2}(t)-1$. Indeed, for $\operatorname{det} t>0$ we have

$$
\begin{gathered}
H_{2, n}^{-2}(t)-1=\frac{1}{2^{2}|\operatorname{det} t|} \times \\
\left(1-2 \operatorname{det} t+|\operatorname{det} t|^{2}+t_{11}^{2}+t_{22}^{2}+\frac{b_{1 n}}{b_{2 n}} t_{12}^{2}+\frac{b_{2 n}}{b_{1 n}} t_{21}^{2}-2\left(t_{11} t_{22}-t_{12} t_{21}\right)\right)= \\
\frac{1}{2^{2}|\operatorname{det} t|}\left[(1-|\operatorname{det} t|)^{2}+\left(t_{11}-t_{22}\right)^{2}+\left(t_{12} \sqrt{\frac{b_{1 n}}{b_{2 n}}}+t_{21} \sqrt{\frac{b_{2 n}}{b_{1 n}}}\right)^{2}\right] .
\end{gathered}
$$

For det $t<0$ we get

$$
\begin{gathered}
H_{2, n}^{-2}(t)-1=\frac{1}{2^{2}|\operatorname{det} t|} \times \\
\left(1+2 \operatorname{det} t+|\operatorname{det} t|^{2}+t_{11}^{2}+t_{22}^{2}+\frac{b_{1 n}}{b_{2 n}} t_{12}^{2}+\frac{b_{2 n}}{b_{1 n}} t_{21}^{2}+2\left(t_{11} t_{22}-t_{12} t_{21}\right)\right)= \\
\frac{1}{2^{2}|\operatorname{det} t|}\left[(1-|\operatorname{det} t|)^{2}+\left(t_{11}+t_{22}\right)^{2}+\left(t_{12} \sqrt{\frac{b_{1 n}}{b_{2 n}}}-t_{21} \sqrt{\frac{b_{2 n}}{b_{1 n}}}\right)^{2}\right] .
\end{gathered}
$$


Using Lemma 4.17, Lemma 4.21 and (4.46) we get

Lemma 4.22. For $t \in G L(2, \mathbb{R})$ we have

$$
\left(\mu_{(b, a)}^{2}\right)^{L_{t}} \perp \mu_{(b, a)}^{2} \quad \text { if } \quad|\operatorname{det} t| \neq 1 .
$$

If $\operatorname{det} t=1$, we have

$$
\left(\mu_{(b, a)}^{2}\right)^{L_{t}} \perp \mu_{(b, a)}^{2} \quad \Leftrightarrow \quad \Sigma^{+}(t)=\Sigma_{1}^{+}(t)+\Sigma_{2}(t)=\infty .
$$

If $\operatorname{det} t=-1$, we have

$$
\left(\mu_{(b, a)}^{2}\right)^{L_{t}} \perp \mu_{(b, a)}^{2} \quad \Leftrightarrow \quad \Sigma^{-}(t)=\Sigma_{1}^{-}(t)+\Sigma_{2}(t)=\infty
$$

where

$$
\begin{gathered}
\Sigma_{1}^{+}(t)=\sum_{n \in \mathbb{Z}}\left[\left(t_{11}-t_{22}\right)^{2}+\left(t_{12} \sqrt{\frac{b_{1 n}}{b_{2 n}}}+t_{21} \sqrt{\frac{b_{2 n}}{b_{1 n}}}\right)^{2}\right], \\
\Sigma_{1}^{-}(t)=\sum_{n \in \mathbb{Z}}\left[\left(t_{11}+t_{22}\right)^{2}+\left(t_{12} \sqrt{\frac{b_{1 n}}{b_{2 n}}}-t_{21} \sqrt{\frac{b_{2 n}}{b_{1 n}}}\right)^{2}\right], \\
\Sigma_{2}\left(t^{-1}\right)=\sum_{n \in \mathbb{Z}}\left[b_{1 n}\left[\left(t_{11}-1\right) a_{1 n}+t_{12} a_{2 n}\right]^{2}+b_{2 n}\left[t_{21} a_{1 n}+\left(t_{22}-1\right) a_{2 n}\right]^{2}\right] .
\end{gathered}
$$

Proof. of Lemma 4.1. We show that it is sufficient to consider only five particular cases:

$$
\begin{gathered}
\exp \left(t E_{12}\right)=I+t E_{12}=\left(\begin{array}{cc}
1 & t \\
0 & 1
\end{array}\right), \quad \exp \left(t E_{21}\right)=I+t E_{21}=\left(\begin{array}{ll}
1 & 0 \\
t & 1
\end{array}\right) \\
\exp \left(t E_{12}\right) P_{1}=\left(\begin{array}{cc}
-1 & t \\
0 & 1
\end{array}\right), \quad \exp \left(t E_{21}\right) P_{2}=\left(\begin{array}{cc}
1 & 0 \\
t & -1
\end{array}\right)
\end{gathered}
$$

and

$$
\tau_{-}(\phi, s)=\left(\begin{array}{cc}
\cos \phi & s^{2} \sin \phi \\
s^{-2} \sin \phi & -\cos \phi
\end{array}\right)
$$

where

$$
P_{1}=\left(\begin{array}{cc}
-1 & 0 \\
0 & 1
\end{array}\right), \quad P_{2}=\left(\begin{array}{cc}
1 & 0 \\
0 & -1
\end{array}\right) \text {. }
$$

We note that $\tau_{-}(\phi, s)=$

$$
\left(\begin{array}{cc}
\cos \phi & s^{2} \sin \phi \\
s^{-2} \sin \phi & -\cos \phi
\end{array}\right)=\left(\begin{array}{cc}
s & 0 \\
0 & s^{-1}
\end{array}\right)\left(\begin{array}{cc}
\cos \phi & -\sin \phi \\
\sin \phi & \cos \phi
\end{array}\right)\left(\begin{array}{cc}
s^{-1} & 0 \\
0 & s
\end{array}\right) P_{2} .
$$


Using Lemma 4.21 we see that we have to consider only two special cases:

$$
t \in G L(2, \mathbb{R}), \quad \operatorname{det} t=1, \quad t_{11}=t_{22},
$$

and

$$
t \in G L(2, \mathbb{R}), \quad \operatorname{det} t=-1, \quad t_{11}=-t_{22} .
$$

In the first case we have

$$
t=\left(\begin{array}{cc}
\alpha & t_{12} \\
t_{21} & \alpha
\end{array}\right), \quad \operatorname{det} t=\alpha^{2}-t_{12} t_{21}=1
$$

In the second case we have

$$
t=\left(\begin{array}{cc}
\alpha & t_{12} \\
t_{21} & -\alpha
\end{array}\right), \quad \operatorname{det} t=-\alpha^{2}-t_{12} t_{21}=-1 .
$$

We can see that in the first (respectively second) case, when $t_{12} t_{21}>0$ (respectively $\left.t_{12} t_{21}<0\right)$, we have $\Sigma_{1}^{+}(t)=\infty$ (respectively $\Sigma_{1}^{-}(t)=\infty$ ).

Indeed, if det $t=1$ and $t_{12} t_{21} \geq 1$, then $\left|t_{21}\right| \geq\left|t_{12}\right|^{-1}$ and we have

$\left|t_{12} \sqrt{\frac{b_{1 n}}{b_{2 n}}}+t_{21} \sqrt{\frac{b_{2 n}}{b_{1 n}}}\right|=\left|t_{12}\right| \sqrt{\frac{b_{1 n}}{b_{2 n}}}+\left|t_{21}\right| \sqrt{\frac{b_{2 n}}{b_{1 n}}} \geq\left|t_{12}\right| \sqrt{\frac{b_{1 n}}{b_{2 n}}}+\left|t_{12}\right|^{-1} \sqrt{\frac{b_{2 n}}{b_{1 n}}} \geq 2$.

When $\operatorname{det} t=1$ and $t_{12} t_{21} \in(0,1)$, then $\left|t_{12}\right|^{-1}>\left|t_{21}\right|$ and we get

$$
\left|t_{12} \sqrt{\frac{b_{1 n}}{b_{2 n}}}+t_{21} \sqrt{\frac{b_{2 n}}{b_{1 n}}}\right|=t_{12} t_{21}\left(\left|t_{21}\right|^{-1} \sqrt{\frac{b_{1 n}}{b_{2 n}}}+\left|t_{12}\right|^{-1} \sqrt{\frac{b_{2 n}}{b_{1 n}}}\right) \geq 2\left|t_{12} t_{21}\right| .
$$

The same is true for the second case, i.e., when det $t=-1$ and $t_{12} t_{21}<0$.

When

$$
\operatorname{det} t=\alpha^{2}-t_{12} t_{21}=1, \quad \text { and } \quad t_{12} t_{21}=0,
$$

we have four cases

$$
\left(\begin{array}{ll}
1 & t \\
0 & 1
\end{array}\right), \quad\left(\begin{array}{ll}
1 & 0 \\
t & 1
\end{array}\right), \quad\left(\begin{array}{cc}
-1 & t \\
0 & -1
\end{array}\right), \quad\left(\begin{array}{cc}
-1 & 0 \\
t & -1
\end{array}\right), \quad t \in \mathbb{R} .
$$

When

$$
\operatorname{det} t=-\alpha^{2}-t_{12} t_{21}=-1, \quad \text { and } \quad t_{12} t_{21}=0 \text {, }
$$


we also have four cases:

$$
\left(\begin{array}{cc}
-1 & t \\
0 & 1
\end{array}\right), \quad\left(\begin{array}{cc}
1 & 0 \\
t & -1
\end{array}\right), \quad\left(\begin{array}{cc}
1 & t \\
0 & -1
\end{array}\right), \quad\left(\begin{array}{cc}
-1 & 0 \\
t & 1
\end{array}\right), \quad t \in \mathbb{R} .
$$

Thus, it remains to consider two cases:

$$
\begin{gathered}
\operatorname{det} t=\alpha^{2}-t_{12} t_{21}=1, \quad \text { and } \quad t_{12} t_{21} \in[-1,0), \\
\operatorname{det} t=-\alpha^{2}-t_{12} t_{21}=-1, \quad \text { and } \quad t_{12} t_{21} \in(0,1] .
\end{gathered}
$$

Finally, we can set in the first case $\alpha=\cos \phi$ since $\alpha^{2}=1+t_{12} t_{21} \in[0,1)$. Then $-t_{12} t_{21}=\sin ^{2} \phi$ so, $t_{12}=-s^{2} \sin \phi$ and $t_{21}=s^{-2} \sin \phi$, with $s>0$.

In the second case we can set $\alpha=\cos \phi$ since $\alpha^{2}=1-t_{12} t_{21} \in[0,1)$. Then $t_{12} t_{21}=\sin ^{2} \phi$ so $t_{12}=s^{2} \sin \phi$ and $t_{21}=s^{-2} \sin \phi$, with $s>0$. Finally, in the first (the second) case we have to consider $t=\tau_{+}(\phi, s)=\left(\begin{array}{cc}\cos \phi & -s^{2} \sin \phi \\ s^{-2} \sin \phi & \cos \phi\end{array}\right), t=\tau_{-}(\phi, s)=\left(\begin{array}{cc}\cos \phi & s^{2} \sin \phi \\ s^{-2} \sin \phi & -\cos \phi\end{array}\right)$.

We show that only the first two cases in (4.61) and (4.62) and the second case in 4.63) are independent. Indeed, we have for $a=\left(\begin{array}{l}a_{1 n} \\ a_{2 n}\end{array}\right)$ (see Lemma 4.22)

$$
\begin{gathered}
t=\left(\begin{array}{ll}
1 & t \\
0 & 1
\end{array}\right),\left(t^{-1}-I\right) a=\left(\left(\begin{array}{cc}
1 & -t \\
0 & 1
\end{array}\right)-I\right)\left(\begin{array}{c}
a_{1 n} \\
a_{2 n}
\end{array}\right)=\left(\begin{array}{c}
-t a_{2 n} \\
0
\end{array}\right), \\
t=\left(\begin{array}{ll}
1 & 0 \\
t & 1
\end{array}\right), \quad\left(t^{-1}-I\right) a=\left(\left(\begin{array}{cc}
1 & 0 \\
-t & 1
\end{array}\right)-I\right)\left(\begin{array}{c}
a_{1 n} \\
a_{2 n}
\end{array}\right)=\left(\begin{array}{c}
0 \\
-t a_{1 n}
\end{array}\right), \\
t=\left(\begin{array}{cc}
-1 & t \\
0 & 1
\end{array}\right)=\left(\begin{array}{ll}
1 & t \\
0 & 1
\end{array}\right)\left(\begin{array}{cc}
-1 & 0 \\
0 & 1
\end{array}\right), \quad t^{-1}=\left(\begin{array}{cc}
-1 & 0 \\
0 & 1
\end{array}\right)\left(\begin{array}{cc}
1 & -t \\
0 & 1
\end{array}\right)= \\
t=\left(\begin{array}{cc}
-1 & t \\
0 & 1
\end{array}\right), \quad\left(\begin{array}{cc}
1 & 0 \\
t & -1
\end{array}\right)=\left(\begin{array}{ll}
1 & 0 \\
t & 1
\end{array}\right) a=\left(\begin{array}{cc}
-2 & t \\
0 & 0
\end{array}\right)\left(\begin{array}{c}
a_{1 n} \\
a_{2 n}
\end{array}\right)=\left(\begin{array}{cc}
-2 a_{1 n}+t a_{2 n} \\
0 & -1
\end{array}\right), \quad t^{-1}=\left(\begin{array}{cc}
1 & 0 \\
0 & -1
\end{array}\right)\left(\begin{array}{cc}
1 & 0 \\
-t & 1
\end{array}\right)= \\
\left(\begin{array}{cc}
1 & 0 \\
t & -1
\end{array}\right), \quad\left(t^{-1}-I\right) a=\left(\begin{array}{cc}
0 & 0 \\
t & -2
\end{array}\right)\left(\begin{array}{l}
a_{1 n} \\
a_{2 n}
\end{array}\right)=\left(\begin{array}{c}
t a_{1 n}-2 a_{2 n}
\end{array}\right) .
\end{gathered}
$$


Therefore, we get

$$
\begin{gathered}
\Sigma^{+}\left(\begin{array}{ll}
1 & t \\
0 & 1
\end{array}\right)=t^{2} \sum_{n \in \mathbb{Z}} b_{1 n}\left(\frac{1}{b_{2 n}}+a_{2 n}^{2}\right) \simeq S_{12}^{L}(\mu)=\sum_{n \in \mathbb{Z}} \frac{b_{1 n}}{2}\left(\frac{1}{2 b_{2 n}}+a_{2 n}^{2}\right), t \neq 0, \\
\Sigma^{+}\left(\begin{array}{ll}
1 & 0 \\
t & 1
\end{array}\right)=t^{2} \sum_{n \in \mathbb{Z}} b_{2 n}\left(\frac{1}{b_{1 n}}+a_{1 n}^{2}\right) \simeq S_{21}^{L}(\mu)=\sum_{n \in \mathbb{Z}} \frac{b_{2 n}}{2}\left(\frac{1}{2 b_{1 n}}+a_{1 n}^{2}\right), t \neq 0, \\
\Sigma^{-}\left(\begin{array}{cc}
-1 & t \\
0 & 1
\end{array}\right)=t^{2} \sum_{n \in \mathbb{Z}} \frac{b_{1 n}}{b_{2 n}}+\sum_{n \in \mathbb{Z}} b_{1 n}\left(-2 a_{1 n}+t a_{2 n}\right)^{2}=: S_{12}^{L,-}(\mu, t), \\
\Sigma^{-}\left(\begin{array}{cc}
1 & 0 \\
t & -1
\end{array}\right)=t^{2} \sum_{n \in \mathbb{Z}} \frac{b_{2 n}}{b_{1 n}}+\sum_{n \in \mathbb{Z}} b_{2 n}\left(t a_{1 n}-2 a_{2 n}\right)^{2}=: S_{21}^{L,-}(\mu, t) .
\end{gathered}
$$

For the last two cases in (4.61) and (4.62) we get respectively

$$
\begin{aligned}
& t=\left(\begin{array}{cc}
-1 & t \\
0 & -1
\end{array}\right)=\left(\begin{array}{cc}
-1 & 0 \\
0 & -1
\end{array}\right)\left(\begin{array}{cc}
1 & -t \\
0 & 1
\end{array}\right), \quad t^{-1}=\left(\begin{array}{cc}
1 & t \\
0 & 1
\end{array}\right)\left(\begin{array}{cc}
-1 & 0 \\
0 & -1
\end{array}\right)= \\
& \left(\begin{array}{cc}
-1 & -t \\
0 & -1
\end{array}\right), \quad\left(t^{-1}-I\right) a=\left(\begin{array}{cc}
-2 & -t \\
0 & -2
\end{array}\right)\left(\begin{array}{l}
a_{1 n} \\
a_{2 n}
\end{array}\right)=-\left(\begin{array}{c}
2 a_{1 n}+t a_{2 n} \\
2 a_{2 n}
\end{array}\right), \\
& t=\left(\begin{array}{cc}
-1 & 0 \\
t & -1
\end{array}\right)=\left(\begin{array}{cc}
-1 & 0 \\
0 & -1
\end{array}\right)\left(\begin{array}{cc}
1 & 0 \\
-t & 1
\end{array}\right), \quad t^{-1}=\left(\begin{array}{ll}
1 & 0 \\
t & 1
\end{array}\right)\left(\begin{array}{cc}
-1 & 0 \\
0 & -1
\end{array}\right) \\
& =\left(\begin{array}{cc}
-1 & 0 \\
-t & -1
\end{array}\right), \quad\left(t^{-1}-I\right) a=\left(\begin{array}{cc}
-2 & 0 \\
-t & -2
\end{array}\right)\left(\begin{array}{c}
a_{1 n} \\
a_{2 n}
\end{array}\right)=-\left(\begin{array}{c}
2 a_{1 n} \\
t a_{1 n}+2 a_{2 n}
\end{array}\right) . \\
& t=\left(\begin{array}{cc}
1 & t \\
0 & -1
\end{array}\right)=\left(\begin{array}{cc}
1 & 0 \\
0 & -1
\end{array}\right)\left(\begin{array}{ll}
1 & t \\
0 & 1
\end{array}\right), \quad t^{-1}=\left(\begin{array}{cc}
1 & -t \\
0 & 1
\end{array}\right)\left(\begin{array}{cc}
1 & 0 \\
0 & -1
\end{array}\right)= \\
& \left(\begin{array}{cc}
1 & t \\
0 & -1
\end{array}\right), \quad\left(t^{-1}-I\right) a=\left(\begin{array}{cc}
0 & t \\
0 & -2
\end{array}\right)\left(\begin{array}{c}
a_{1 n} \\
a_{2 n}
\end{array}\right)=\left(\begin{array}{c}
t a_{2 n} \\
-2 a_{2 n}
\end{array}\right), \\
& t=\left(\begin{array}{cc}
-1 & 0 \\
t & 1
\end{array}\right)=\left(\begin{array}{cc}
-1 & 0 \\
0 & 1
\end{array}\right)\left(\begin{array}{ll}
1 & 0 \\
t & 1
\end{array}\right), \quad t^{-1}=\left(\begin{array}{cc}
1 & 0 \\
-t & 1
\end{array}\right)\left(\begin{array}{cc}
-1 & 0 \\
0 & 1
\end{array}\right)= \\
& \left(\begin{array}{cc}
-1 & 0 \\
t & 1
\end{array}\right), \quad\left(t^{-1}-I\right) a=\left(\begin{array}{cc}
-2 & 0 \\
t & 0
\end{array}\right)\left(\begin{array}{c}
a_{1 n} \\
a_{2 n}
\end{array}\right)=\left(\begin{array}{c}
-2 a_{1 n} \\
t a_{1 n}
\end{array}\right) .
\end{aligned}
$$

Set

$$
S_{11}^{L}(\mu):=S_{12}^{L,-}(\mu, 0)=4 \sum_{n \in \mathbb{Z}} b_{1 n} a_{1 n}^{2}, \quad S_{22}^{L}(\mu):=S_{21}^{L,-}(\mu, 0)=4 \sum_{n \in \mathbb{Z}} b_{2 n} a_{2 n}^{2} .
$$


With this notation we see that the second two cases in (4.61) and (4.62) are dependent:

$$
\begin{aligned}
& \Sigma^{+}\left(\begin{array}{cc}
-1 & t \\
0 & -1
\end{array}\right)=t^{2} \sum_{n \in \mathbb{Z}} \frac{b_{1 n}}{b_{2 n}}+\sum_{n \in \mathbb{Z}}\left[b_{1 n}\left(-2 a_{1 n}-t a_{2 n}\right)^{2}+b_{2 n}\left(-2 a_{2 n}\right)^{2}\right] \\
= & S_{12}^{L,-}(\mu,-t)+S_{22}^{L}(\mu), \quad \text { note that }\left(\begin{array}{cc}
-1 & t \\
0 & -1
\end{array}\right)=\left(\begin{array}{cc}
1 & 0 \\
0 & -1
\end{array}\right)\left(\begin{array}{cc}
-1 & t \\
0 & 1
\end{array}\right) . \\
& \Sigma^{+}\left(\begin{array}{cc}
-1 & 0 \\
t & -1
\end{array}\right)=t^{2} \sum_{n \in \mathbb{Z}} \frac{b_{2 n}}{b_{1 n}}+\sum_{n \in \mathbb{Z}}\left[b_{1 n}\left(-2 a_{1 n}\right)^{2}+b_{2 n}\left(-t a_{1 n}-2 a_{2 n}\right)^{2}\right] \\
= & S_{21}^{L,-}(\mu,-t)+S_{11}^{L}(\mu), \quad \text { note that }\left(\begin{array}{cc}
-1 & 0 \\
t & -1
\end{array}\right)=\left(\begin{array}{cc}
-1 & 0 \\
0 & 1
\end{array}\right)\left(\begin{array}{cc}
1 & 0 \\
t & -1
\end{array}\right) . \\
\Sigma^{-} & \left(\begin{array}{cc}
1 & t \\
0 & -1
\end{array}\right)=t^{2} \sum_{n \in \mathbb{Z}} \frac{b_{1 n}}{b_{2 n}}+t^{2} \sum_{n \in \mathbb{Z}} b_{1 n} a_{2 n}^{2}+4 \sum_{n \in \mathbb{Z}} b_{2 n} a_{2 n}^{2} \simeq t^{2} S_{12}^{L}(\mu)+S_{22}^{L}(\mu), \\
\Sigma^{-} & \left(\begin{array}{cc}
-1 & 0 \\
t & 1
\end{array}\right)=t^{2} \sum_{n \in \mathbb{Z}} \frac{b_{2 n}}{b_{1 n}}+4 \sum_{n \in \mathbb{Z}} b_{1 n} a_{1 n}^{2}+t^{2} \sum_{n \in \mathbb{Z}} b_{2 n} a_{1 n}^{2} \simeq t^{2} S_{21}^{L}(\mu)+S_{11}^{L}(\mu) .
\end{aligned}
$$

To compare $\left(\mu_{(b, a)}^{2}\right)^{L_{\tau_{ \pm}(\phi, s)}}$ and $\mu_{(b, a)}^{2}$ we calculate $\tau_{+}^{-1}(\phi, s)$ and $\tau_{-}^{-1}(\phi, s)$. Since

$$
\tau_{+}(\phi, s)=\left(\begin{array}{cc}
\cos \phi & -s^{2} \sin \phi \\
s^{-2} \sin \phi & \cos \phi
\end{array}\right), \quad \tau_{-}(\phi, s)=\left(\begin{array}{cc}
\cos \phi & s^{2} \sin \phi \\
s^{-2} \sin \phi & -\cos \phi
\end{array}\right),
$$

we get

$$
\begin{aligned}
& \tau_{+}^{-1}(\phi, s)=\left(\begin{array}{cc}
\cos \phi & s^{2} \sin \phi \\
-s^{-2} \sin \phi & \cos \phi
\end{array}\right), \quad \tau_{-}^{-1}(\phi, s)=\left(\begin{array}{cc}
\cos \phi & s^{2} \sin \phi \\
s^{-2} \sin \phi & -\cos \phi
\end{array}\right) \\
&= \tau_{-}(\phi, s) . \text { Since } \tau_{+}^{-1}(\phi, s)-I= \\
&\left(\begin{array}{cc}
\cos \phi-1 & s^{2} \sin \phi \\
-s^{-2} \sin \phi & \cos \phi-1
\end{array}\right)=\left(\begin{array}{cc}
-2 \sin ^{2} \frac{\phi}{2} & s^{2} 2 \sin \frac{\phi}{2} \cos \frac{\phi}{2} \\
-s^{-2} 2 \sin \frac{\phi}{2} \cos \frac{\phi}{2} & -2 \sin ^{2} \frac{\phi}{2}
\end{array}\right)= \\
&\left(\begin{array}{cc}
-2 \sin \frac{\phi}{2} & 0 \\
0 & -2 \sin \frac{\phi}{2}
\end{array}\right)\left(\begin{array}{cc}
\sin \frac{\phi}{2} & -s^{2} \cos \frac{\phi}{2} \\
s^{-2} \cos \frac{\phi}{2} & \sin \frac{\phi}{2}
\end{array}\right)
\end{aligned}
$$


and $\tau_{-}^{-1}(\phi, s)-I=$

$$
\begin{gathered}
\left(\begin{array}{cc}
\cos \phi-1 & s^{2} \sin \phi \\
s^{-2} \sin \phi & -\cos \phi-1
\end{array}\right)=\left(\begin{array}{cc}
-2 \sin ^{2} \frac{\phi}{2} & s^{2} 2 \sin \frac{\phi}{2} \cos \frac{\phi}{2} \\
s^{-2} 2 \sin \frac{\phi}{2} \cos \frac{\phi}{2} & -2 \cos ^{2} \frac{\phi}{2}
\end{array}\right)= \\
\left(\begin{array}{cc}
-2 \sin \frac{\phi}{2} & 0 \\
0 & -2 \cos \frac{\phi}{2}
\end{array}\right)\left(\begin{array}{cc}
\sin \frac{\phi}{2} & -s^{2} \cos \frac{\phi}{2} \\
-s^{-2} \sin \frac{\phi}{2} & \cos \frac{\phi}{2}
\end{array}\right),
\end{gathered}
$$

we have (see (4.60)

$$
\begin{gathered}
\Sigma_{2}\left(\tau_{-}(\phi, s)\right)=4 \sin ^{2} \frac{\phi}{2} \sum_{n \in \mathbb{Z}} b_{1 n}\left(\sin \frac{\phi}{2} a_{1 n}-s^{2} \cos \frac{\phi}{2} a_{2 n}\right)^{2}+ \\
4 \cos ^{2} \frac{\phi}{2} \sum_{n \in \mathbb{Z}} b_{2 n}\left(-s^{-2} \sin \frac{\phi}{2} a_{1 n}+\cos \frac{\phi}{2} a_{2 n}\right)^{2} \\
\sim \sum_{n \in \mathbb{Z}}\left(4 \sin ^{2} \frac{\phi}{2} b_{1 n}+4 \cos ^{2} \frac{\phi}{2} s^{-2} b_{2 n}\right)\left(\sin \frac{\phi}{2} a_{1 n}-s^{2} \cos \frac{\phi}{2} a_{2 n}\right)^{2} \\
\Sigma_{2}\left(\tau_{-}(\phi, s)\right)=\sum_{n \in \mathbb{Z}}\left(4 \sin ^{2} \frac{\phi}{2} b_{1 n}+4 \cos ^{2} \frac{\phi}{2} s^{-2} b_{2 n}\right)\left(\sin \frac{\phi}{2} a_{1 n}-s^{2} \cos \frac{\phi}{2} a_{2 n}\right)^{2} .
\end{gathered}
$$

Finally, for $t=\tau_{-}(\phi, s)$ we get

$$
\mu^{L_{\tau_{-}(\phi, s)}} \perp \mu \Leftrightarrow \sin ^{2} \phi \Sigma_{1}(s)+\Sigma_{2}\left(\tau_{-}(\phi, s)\right)=\infty,
$$

where

$$
\Sigma_{1}(s)=\sum_{n \in \mathbb{Z}}\left(s^{2} \sqrt{\frac{b_{1 n}}{b_{2 n}}}-s^{-2} \sqrt{\frac{b_{2 n}}{b_{1 n}}}\right)^{2} .
$$

We have for $t=\tau_{+}(\phi, s)($ see (4.60) $)$

$$
\mu^{L_{\tau_{+}(\phi, s)}} \perp \mu \Leftrightarrow \sin ^{2} \phi \Sigma_{1}(s)+\Sigma_{2}\left(\tau_{+}(\phi, s)\right)=\infty,
$$

where $\Sigma_{2}\left(\tau_{+}(\phi, s)\right)=$

$$
4 \sin ^{2} \frac{\phi}{2} \sum_{n \in \mathbb{Z}}\left[b_{1 n}\left(\sin \frac{\phi}{2} a_{1 n}-s^{2} \cos \frac{\phi}{2} a_{2 n}\right)^{2}+b_{2 n}\left(s^{-2} \cos \frac{\phi}{2} a_{1 n}+\sin \frac{\phi}{2} a_{2 n}\right)^{2}\right] \text {. }
$$


We show that the condition $\mu^{L_{\tau_{+}(\phi, s)}} \perp \mu$ depends on the previous conditions of the orthogonality. Indeed, for $t=\tau_{-}(\phi, s)$ we have

$\mu^{L_{\tau_{-}(\phi, s)}} \perp \mu \Leftrightarrow(a) \Sigma_{1}(s)=\infty \quad$ or $\quad(b) \Sigma_{1}(s)<\infty$, but $\Sigma_{2}\left(\tau_{-}(\phi, s)\right)=\infty$.

For $t=\tau_{+}(\phi, s)$ we get respectively

$\mu^{L_{\tau_{+}(\phi, s)}} \perp \mu \Leftrightarrow(c) \Sigma_{1}(s)=\infty \quad$ or $\quad(d) \Sigma_{1}(s)<\infty$, but $\Sigma_{2}\left(\tau_{+}(\phi, s)\right)=\infty$.

We see that $(c) \Leftrightarrow(a)$. To investigate the condition $(d)$ we observe that if $\Sigma_{1}(s)<\infty$, then $\lim _{n \rightarrow \infty} s^{2} \sqrt{\frac{b_{1 n}}{b_{2 n}}}=1$ therefore, we have $b_{2 n} \sim s^{4} b_{1 n}$ hence, the following equivalence holds: $\Sigma_{2}\left(\tau_{+}(\phi, s)\right)=$

$$
\begin{gathered}
4 \sin ^{2} \frac{\phi}{2} \sum_{n \in \mathbb{Z}}\left[b_{1 n}\left(\sin \frac{\phi}{2} a_{1 n}-s^{2} \cos \frac{\phi}{2} a_{2 n}\right)^{2}+b_{2 n}\left(s^{-2} \cos \frac{\phi}{2} a_{1 n}+\sin \frac{\phi}{2} a_{2 n}\right)^{2}\right] \\
\sim 4 \sin ^{2} \frac{\phi}{2} \sum_{n \in \mathbb{Z}}\left[b_{1 n}\left(\sin \frac{\phi}{2} a_{1 n}-s^{2} \cos \frac{\phi}{2} a_{2 n}\right)^{2}+b_{1 n}\left(\cos \frac{\phi}{2} a_{1 n}+s^{2} \sin \frac{\phi}{2} a_{2 n}\right)^{2}\right]= \\
4 \sin ^{2} \frac{\phi}{2} \sum_{n \in \mathbb{Z}} b_{1 n}\left[a_{1 n}^{2}+s^{4} a_{2 n}^{2}\right] \sim 4 \sin ^{2} \frac{\phi}{2} \sum_{n \in \mathbb{Z}}\left(b_{1 n} a_{1 n}^{2}+b_{2 n} a_{2 n}^{2}\right)= \\
\sin ^{2} \frac{\phi}{2}\left[S_{11}^{L}(\mu)+S_{22}^{L}(\mu)\right] .
\end{gathered}
$$

We see that condition $(d)$ follows from the conditions $S_{11}^{L}(\mu)=S_{12}^{L,-}(\mu, 0)=$ $\infty$ and $S_{22}^{L}(\mu)=S_{21}^{L,-}(\mu, 0)=\infty$. This completes the proof of Lemma 4.1.

4.4. The explicit expression for $\left(D^{-1}(\lambda) \mu, \mu\right)$

The following lemma will be systematically used in what follows.

Lemma 4.23. For the matrix $D\left(\lambda_{1}, \lambda_{2}, \ldots, \lambda_{m}\right)$ defined below

$$
D\left(\lambda_{1}, \lambda_{2}, \ldots, \lambda_{m}\right)=\left(\begin{array}{cccc}
1+\lambda_{1} & 1 & \ldots & 1 \\
1 & 1+\lambda_{2} & \ldots & 1 \\
& & \ldots & \\
1 & 1 & \ldots & 1+\lambda_{m}
\end{array}\right)
$$

and $\mu=\left(\mu_{k}\right)_{k=1}^{m} \in \mathbb{R}^{m}$ we have

$$
\left(D^{-1}\left(\lambda_{1}, \lambda_{2}, \ldots, \lambda_{m}\right) \mu, \mu\right)=\frac{\sum_{k=1}^{m} \frac{\mu_{k}^{2}}{\lambda_{k}}+\sum_{1 \leq k<n \leq m} \frac{\left(\mu_{k}-\mu_{n}\right)^{2}}{\lambda_{k} \lambda_{n}}}{1+\sum_{k=1}^{m} \frac{1}{\lambda_{k}}} .
$$


Proof. Let us set $d_{m}\left(\lambda_{1}, \lambda_{2}, \ldots, \lambda_{m}\right)=\operatorname{det}\left(D\left(\lambda_{1}, \lambda_{2}, \ldots, \lambda_{m}\right)\right)$. It is easy to see that

$$
d_{m}\left(\lambda_{1}, \lambda_{2}, \ldots, \lambda_{m}\right)=\prod_{k=1}^{m} \lambda_{k}\left(1+\sum_{k=1}^{m} \frac{1}{\lambda_{k}}\right) .
$$

For arbitrary $m$ we have

$$
D^{-1}\left(\lambda_{1}, \lambda_{2}, \ldots, \lambda_{m}\right)=\left(\begin{array}{cccc}
1+\lambda_{1} & 1 & \ldots & 1 \\
1 & 1+\lambda_{2} & \ldots & 1 \\
& & \ldots & \\
1 & 1 & \ldots & 1+\lambda_{m}
\end{array}\right)^{-1}=\left(D_{k n}^{-1}\right)_{k, n=1}^{m},
$$

where

$$
\begin{aligned}
& D_{n n}^{-1}=\frac{d_{m-1}\left(\lambda_{1}, \ldots, \hat{\lambda}_{n}, \ldots \lambda_{m}\right)}{d_{m}\left(\lambda_{1}, \lambda_{2}, \ldots, \lambda_{m}\right)}=\left(1+\sum_{k=1}^{m} \frac{1}{\lambda_{k}}\right)^{-1} \frac{1}{\lambda_{n}}\left(1+\sum_{k=1, k \neq n}^{m} \frac{1}{\lambda_{k}}\right), \\
& D_{k n}^{-1}=\frac{-\left.d_{m-1}\left(\lambda_{1}, \ldots, \hat{\lambda}_{n}, \ldots \lambda_{m}\right)\right|_{\lambda_{k}=0}}{d_{m}\left(\lambda_{1}, \lambda_{2}, \ldots, \lambda_{m}\right)}=-\frac{1}{\lambda_{k} \lambda_{n}}\left(1+\sum_{k=1}^{m} \frac{1}{\lambda_{k}}\right)^{-1}, k \neq n,
\end{aligned}
$$

since using (4.71) we have

$$
\left.d_{m-1}\left(\lambda_{1}, \ldots, \hat{\lambda}_{n}, \ldots \lambda_{m}\right)\right|_{\lambda_{k}=0}=\lim _{\lambda_{k} \rightarrow 0} \prod_{p=1, p \neq n}^{m} \lambda_{p}\left(1+\sum_{p=1, p \neq n}^{m} \frac{1}{\lambda_{p}}\right)=\frac{1}{\lambda_{k} \lambda_{n}} \prod_{p=1}^{m} \lambda_{p} .
$$

Finally, we have for $\mu=\left(\mu_{1}, \mu_{2}, \ldots, \mu_{m}\right) \in \mathbb{R}^{m}$

$$
\begin{gathered}
\left(D^{-1}\left(\lambda_{1}, \lambda_{2}, \ldots, \lambda_{m}\right) \mu, \mu\right)=\sum_{k, n=1}^{m} D_{k n}^{-1} \mu_{k} \mu_{n}= \\
\left(1+\sum_{k=1}^{m} \frac{1}{\lambda_{k}}\right)^{-1}\left[\sum_{n=1}^{m} \frac{\mu_{n}^{2}}{\lambda_{n}}\left(1+\sum_{k=1, k \neq n}^{m} \frac{1}{\lambda_{k}}\right)-2 \sum_{1 \leq k<n \leq m} \frac{\mu_{k} \mu_{n}}{\lambda_{k} \lambda_{n}}\right]= \\
\left(1+\sum_{k=1}^{m} \frac{1}{\lambda_{k}}\right)^{-1}\left[\sum_{n=1}^{m} \frac{\mu_{n}^{2}}{\lambda_{n}}+\sum_{1 \leq k<n \leq m} \frac{\left(\mu_{k}-\mu_{n}\right)^{2}}{\lambda_{k} \lambda_{n}}\right]
\end{gathered}
$$


Remark 4.7. Some useful observations. If we set $f_{(m)}=\left(f_{k}\right)_{k=1}^{m}$ and $g_{(m)}$ $=\left(g_{k}\right)_{k=1}^{m}$ where $f_{k}=\frac{\mu_{k}}{\sqrt{\lambda_{k}}}$ and $g_{k}=\frac{1}{\sqrt{\lambda_{k}}}$ we can recognize that

$$
\sum_{n=1}^{m} \frac{\mu_{n}^{2}}{\lambda_{n}}=\left\|f_{(m)}\right\|^{2}=\Gamma\left(f_{(m)}\right)
$$

and

$$
\sum_{1 \leq k<n \leq m} \frac{\left(\mu_{k}-\mu_{n}\right)^{2}}{\lambda_{k} \lambda_{n}}=\sum_{1 \leq k<n \leq m}\left|\begin{array}{ll}
f_{k} & f_{n} \\
g_{k} & g_{n}
\end{array}\right|^{2}=\Gamma\left(f_{(m)}, g_{(m)}\right)
$$

since

$$
\left|\begin{array}{ll}
f_{k} & f_{n} \\
g_{k} & g_{n}
\end{array}\right|^{2}=\left|\begin{array}{cc}
\frac{\mu_{k}}{\sqrt{\lambda_{k}}} & \frac{\mu_{n}}{\sqrt{\lambda_{n}}} \\
\frac{1}{\sqrt{\lambda_{k}}} & \frac{1}{\sqrt{\lambda_{n}}}
\end{array}\right|^{2}=\frac{\left(\mu_{k}-\mu_{n}\right)^{2}}{\lambda_{k} \lambda_{n}} .
$$

Set $\Delta(f, g)=\frac{\Gamma(f)+\Gamma(f, g)}{\Gamma(g)+1}$ for two vectors $f$ and $g$. Finally, we get

$$
\left.\left(D^{-1}\left(\lambda_{1}, \lambda_{2}, \ldots, \lambda_{m}\right) \mu, \mu\right)=\Delta\left(f_{(m)}, g_{(m)}\right)\right)=\frac{\Gamma\left(f_{(m)}\right)+\Gamma\left(f_{(m)}, g_{(m)}\right)}{\Gamma\left(g_{(m)}\right)+1} .
$$

where $\Gamma\left(f_{1}, f_{2}, \ldots, f_{n}\right)$ is the Gram determinant and $\gamma\left(f_{1}, f_{2}, \ldots, f_{n}\right)$ is the Gram matrix of $n$ vectors $f_{1}, f_{2}, \ldots, f_{n}$ in a Hilbert space (see [7]).

4.5. The proof of Lemmas 4.2-4.7

Proof. The proof of Lemma 4.4 is based on Lemma 4.23. We find out when the inclusion

$$
D_{1 n} \mathbf{1} \in\left\langle A_{k n} \mathbf{1}=\left(x_{1 k} D_{1 n}+x_{2 k} D_{2 n}\right) \mathbf{1} \mid k \in \mathbb{Z}\right\rangle
$$

holds. Fix $m \in \mathbb{N}$, since $M x_{1 k}=a_{1 k}$, we put $\sum_{k=-m}^{m} t_{k} a_{1 k}=(t, b)=1$, where $t=\left(t_{k}\right)_{k=-m}^{m}$ and $b=\left(a_{1 k}\right)_{k=-m}^{m}$. We have

$$
\begin{gathered}
\left\|\left[\sum_{k=-m}^{m} t_{k}\left(x_{1 k} D_{1 n}+x_{2 k} D_{2 n}\right)-D_{1 n}\right] \mathbf{1}\right\|^{2}= \\
\left\|\sum_{k=-m}^{m} t_{k}\left[\left(x_{1 k}-a_{1 k}\right) D_{1 n}+x_{2 k} D_{2 n}\right] \mathbf{1}\right\|^{2}=\sum_{-m \leq k, r \leq m}\left(f_{k}, f_{r}\right) t_{k} t_{r}=:\left(A_{2 m+1} t, t\right),
\end{gathered}
$$


where $A_{2 m+1}=\left(\left(f_{k}, f_{r}\right)\right)_{k, r=-m}^{m}$, and $f_{k}=\left[\left(x_{1 k}-a_{1 k}\right) D_{1 n}+x_{2 k} D_{2 n}\right] \mathbf{1}$. We have

$$
\begin{gathered}
\left(f_{k}, f_{k}\right)=\left\|\left[\left(x_{1 k}-a_{1 k}\right) D_{1 n}+x_{2 k} D_{2 n}\right] \mathbf{1}\right\|^{2}=\frac{1}{2 b_{1 k}} \frac{b_{1 n}}{2}+\left(\frac{1}{2 b_{2 k}}+a_{2 k}^{2}\right) \frac{b_{2 n}}{2} \sim \\
\frac{1}{2 b_{1 k}}+\frac{1}{2 b_{2 k}}+a_{2 k}^{2}, \\
\left(f_{k}, f_{r}\right)=\left(\left[\left(x_{1 k}-a_{1 k}\right) D_{1 n}+x_{2 k} D_{2 n}\right] \mathbf{1},\left[\left(x_{1 r}-a_{1 r}\right) D_{1 n}+x_{2 r} D_{2 n}\right] \mathbf{1}\right)= \\
\left(x_{2 k}, x_{2 r}\right)\left(D_{2 n} \mathbf{1}, D_{2 n} \mathbf{1}\right)=a_{2 k} a_{2 r} \frac{b_{2 n}}{2} \simeq a_{2 k} a_{2 r} .
\end{gathered}
$$

Finally, we have

$$
\left(f_{k}, f_{k}\right) \sim \frac{1}{2 b_{1 k}}+\frac{1}{2 b_{2 k}}+a_{2 k}^{2}, \quad\left(f_{k}, f_{r}\right) \sim a_{2 k} a_{2 r}, \quad k \neq r .
$$

For $A_{(m)}=\left(\left(f_{k}, f_{r}\right)\right)_{k, r=1}^{m}$, and $b=\left(a_{11}, a_{12}, \ldots, a_{1 m}\right) \in \mathbb{R}^{m}$ we have

$$
\begin{aligned}
& A_{(m)}=\gamma\left(f_{1}, f_{2}, \ldots, f_{m}\right)=\left(\begin{array}{llll}
\left(f_{1}, f_{1}\right) & \left(f_{1}, f_{2}\right) & \ldots & \left(f_{1}, f_{m}\right) \\
\left(f_{2}, f_{1}\right) & \left(f_{2}, f_{2}\right) & \ldots & \left(f_{2}, f_{m}\right) \\
& & \ldots & \\
\left(f_{m}, f_{1}\right) & \left(f_{m}, f_{2}\right) & \ldots & \left(f_{m}, f_{m}\right)
\end{array}\right)= \\
& \left(\begin{array}{cccc}
\frac{1}{2 b_{11}}+\frac{1}{2 b_{21}}+a_{21}^{2} & a_{21} a_{22} & \cdots & a_{21} a_{2 m} \\
a_{22} a_{21} & \frac{1}{2 b_{12}}+\frac{1}{2 b_{22}}+a_{22}^{2} & \cdots & a_{22} a_{2 m} \\
& & \cdots & \\
a_{2 m} a_{21} & a_{2 m} a_{22} & \cdots & \frac{1}{2 b_{1 m}}+\frac{1}{2 b_{2 m}}+a_{2 m}^{2}
\end{array}\right)= \\
& \left(\begin{array}{cccc}
a_{21} & 0 & \ldots & 0 \\
0 & a_{22} & \ldots & 0 \\
& & \ldots & \\
0 & 0 & \ldots & a_{2 m}
\end{array}\right)\left(\begin{array}{cccc}
1+\lambda_{1} & 1 & \ldots & 1 \\
1 & 1+\lambda_{2} & \ldots & 1 \\
& & \ldots & \\
1 & 1 & \ldots & 1+\lambda_{m}
\end{array}\right)\left(\begin{array}{cccc}
a_{21} & 0 & \ldots & 0 \\
0 & a_{22} & \ldots & 0 \\
& & \ldots & \\
0 & 0 & \ldots & a_{2 m}
\end{array}\right)
\end{aligned}
$$

where $\lambda_{k}=\frac{\frac{1}{2 b_{1 k}}+\frac{1}{2 b_{2 k}}}{a_{2 k}^{2}}, 1 \leq k \leq m$. Using (4.69) we conclude that

$$
A_{(m)}=\operatorname{diag}\left(a_{21}, a_{22}, \ldots, a_{2 m}\right) D\left(\lambda_{1}, \lambda_{2}, \ldots, \lambda_{m}\right) \operatorname{diag}\left(a_{21}, a_{22}, \ldots, a_{2 m}\right) .
$$

Recall that $\mu=\operatorname{diag}\left(a_{21}, a_{22},, \ldots, a_{2 m}\right)^{-1} b=\left(\frac{a_{11}}{a_{21}}, \frac{a_{12}}{a_{22}}, \ldots, \frac{a_{1 m}}{a_{2 m}}\right)$, where $b=$ $\left(a_{11}, a_{12}, \ldots, a_{1 m}\right) \in \mathbb{R}^{m}$, then

$$
\left(A_{(m)}^{-1} b, b\right)=\left(D^{-1}\left(\lambda_{1}, \lambda_{2}, \ldots, \lambda_{m}\right) \mu, \mu\right), \lambda_{k}=\left(\frac{1}{2 b_{1 k}}+\frac{1}{2 b_{2 k}}\right) a_{2 k}^{-2}, \mu_{k}=a_{1 k} a_{2 k}^{-1} .
$$


Using Lemma 4.23 for the operator $A_{2 m+1}$, and the vector $b \in \mathbb{R}^{2 m+1}$ we obtain

$$
\left(A_{2 m+1}^{-1} b, b\right)=\frac{\sum_{k=-m}^{m} \frac{a_{1 k}^{2}}{\frac{1}{2 b_{1 k}}+\frac{1}{2 b_{2 k}}}+\sum_{-m \leq k<n \leq m} \frac{\left(a_{1 k} a_{2 n}-a_{1 n} a_{2 k}\right)^{2}}{\left(\frac{1}{2 b_{1 k}}+\frac{1}{2 b_{2 k}}\right)\left(\frac{1}{2 b_{1 n}}+\frac{1}{2 b_{2 n}}\right)}}{\sum_{k=-m}^{m} \frac{a_{2 k}^{2}}{\frac{1}{2 b_{1 k}}+\frac{1}{2 b_{2 k}}}+1}
$$

$=\Delta\left(f_{(m)}, g_{(m)}\right)$, where

$$
f_{m}=\left(a_{1 k}\left(\frac{1}{2 b_{1 k}}+\frac{1}{2 b_{2 k}}\right)^{-1 / 2}\right)_{k=-m}^{m}, g_{m}=\left(a_{2 k}\left(\frac{1}{2 b_{1 k}}+\frac{1}{2 b_{2 k}}\right)^{-1 / 2}\right)_{k=-m}^{m}
$$

This proves Lemma 4.4

The proof of Lemma 4.5 is exactly the same.

The proof of Lemma 4.2 is also based on Lemma 4.23.

Proof. We study when $x_{1 n} x_{1 t} \in\left\langle A_{n k} A_{t k} \mathbf{1} \mid k \in \mathbb{Z}\right\rangle$. Since

$$
\begin{aligned}
& A_{n k} A_{t k}=\left(x_{1 n} D_{1 k}+x_{2 n} D_{2 k}\right)\left(x_{1 t} D_{1 k}+x_{2 t} D_{2 k}\right)= \\
& x_{1 n} x_{1 t} D_{1 k}^{2}+\left(x_{1 n} x_{2 t}+x_{2 n} x_{1 t}\right) D_{1 k} D_{2 k}+x_{2 n} x_{2 t} D_{2 k}^{2}
\end{aligned}
$$

and $M D_{1 k}^{2} \mathbf{1}=-\frac{b_{1 k}}{2}$, set $-\sum_{k=-m}^{m} t_{k} \frac{b_{1 k}}{2}=\left(t, b^{\prime}\right)=1$, where $t=\left(t_{k}\right)_{k=-m}^{m}$ and $b^{\prime}=-\left(\frac{b_{1 k}}{2}\right)_{k} \sim b=\left(b_{1 k}\right)_{k=-m}^{m}$. We have

$$
\begin{gathered}
\left\|\left[\sum_{k=-m}^{m} t_{k} A_{n k} A_{t k}-x_{1 n} x_{1 t}\right] \mathbf{1}\right\|^{2}= \\
\left\|\sum_{k=-m}^{m} t_{k}\left[x_{1 n} x_{1 t}\left(D_{1 k}^{2}+\frac{b_{1 k}}{2}\right)+\left(x_{1 n} x_{2 t}+x_{2 n} x_{1 t}\right) D_{1 k} D_{2 k}+x_{2 n} x_{2 t} D_{2 k}^{2}\right] \mathbf{1}\right\|^{2} \\
=\sum_{-m \leq k, r \leq m}\left(f_{k}, f_{r}\right) t_{k} t_{r}=:\left(A_{2 m+1} t, t\right),
\end{gathered}
$$

where $A_{2 m+1}=\left(\left(f_{k}, f_{r}\right)\right)_{k, r=-m}^{m}$ and

$$
f_{k}=\left[x_{1 n} x_{1 t}\left(D_{1 k}^{2}+\frac{b_{1 k}}{2}\right)+\left(x_{1 n} x_{2 t}+x_{2 n} x_{1 t}\right) D_{1 k} D_{2 k}+x_{2 n} x_{2 t} D_{2 k}^{2}\right] \mathbf{1}
$$


If we denote by $c_{k n}=\left\|x_{k n}\right\|^{2}=\frac{1}{2 b_{k n}}+a_{k n}^{2}$, we get

$$
\begin{gathered}
\left(f_{k}, f_{k}\right)=\left\|\left[x_{1 n} x_{1 t}\left(D_{1 k}^{2}+\frac{b_{1 k}}{2}\right)+\left(x_{1 n} x_{2 t}+x_{2 n} x_{1 t}\right) D_{1 k} D_{2 k}+x_{2 n} x_{2 t} D_{2 k}^{2}\right] \mathbf{1}\right\|^{2}= \\
c_{1 n} c_{1 t} 2\left(\frac{b_{1 k}}{2}\right)^{2}+\left(c_{1 n} c_{2 t}+c_{1 t} c_{2 n}+2 a_{1 n} a_{2 t} a_{1 t} a_{2 n}\right) \frac{b_{1 k}}{2} \frac{b_{2 k}}{2}+c_{2 n} c_{2 t} 3\left(\frac{b_{2 k}}{2}\right)^{2} \\
\sim\left(b_{1 k}+b_{2 k}\right)^{2}, \quad\left(f_{k}, f_{r}\right)= \\
\left(\left(x_{1 n} x_{1 t}\left(D_{1 k}^{2}+\frac{b_{1 k}}{2}\right)+\left(x_{1 n} x_{2 t}+x_{2 n} x_{1 t}\right) D_{1 k} D_{2 k}+x_{2 n} x_{2 t} D_{2 k}^{2}\right) \mathbf{1}\right. \\
\left.\left(x_{1 n} x_{1 t}\left(D_{1 r}^{2}+\frac{b_{1 r}}{2}\right)+\left(x_{1 n} x_{2 t}+x_{2 n} x_{1 t}\right) D_{1 r} D_{2 r}+x_{2 n} x_{2 t} D_{2 r}^{2}\right) \mathbf{1}\right)= \\
c_{2 n} c_{2 t} \frac{b_{2 k}}{2} \frac{b_{2 r}}{2} \sim b_{2 k} b_{2 r} .
\end{gathered}
$$

Finally, we have

$$
\left(f_{k}, f_{k}\right) \sim\left(b_{1 k}+b_{2 k}\right)^{2}, \quad\left(f_{k}, f_{r}\right) \sim b_{2 k} b_{2 r}, \quad k \neq r .
$$

For $A_{(m)}=\left(\left(f_{k}, f_{r}\right)\right)_{k, r=1}^{m}$, and $b=\left(a_{11}, a_{12}, \ldots, a_{1 m}\right) \in \mathbb{R}^{m}$ we have

$$
\begin{aligned}
& A_{(m)}=\gamma\left(f_{1}, f_{2}, \ldots, f_{m}\right)=\left(\begin{array}{llll}
\left(f_{1}, f_{1}\right) & \left(f_{1}, f_{2}\right) & \ldots & \left(f_{1}, f_{m}\right) \\
\left(f_{2}, f_{1}\right) & \left(f_{2}, f_{2}\right) & \ldots & \left(f_{2}, f_{m}\right) \\
& & \ldots & \\
\left(f_{m}, f_{1}\right) & \left(f_{m}, f_{2}\right) & \ldots & \left(f_{m}, f_{m}\right)
\end{array}\right)= \\
& \left(\begin{array}{cccc}
\left(b_{11}+b_{21}\right)^{2} & b_{21} b_{22} & \ldots & b_{21} b_{2 m} \\
b_{22} b_{21} & \left(b_{12}+b_{22}\right)^{2} & \ldots & b_{22} b_{2 m} \\
& & \ldots & \\
b_{2 m} b_{21} & b_{2 m} b_{22} & \ldots & \left(b_{1 m}+b_{2 m}\right)^{2}
\end{array}\right)= \\
& \left(\begin{array}{cccc}
b_{21} & 0 & \ldots & 0 \\
0 & b_{22} & \ldots & 0 \\
& & \ldots & \\
0 & 0 & \ldots & b_{2 m}
\end{array}\right)\left(\begin{array}{cccc}
1+\lambda_{1} & 1 & \ldots & 1 \\
1 & 1+\lambda_{2} & \ldots & 1 \\
& & \ldots & \\
1 & 1 & \ldots & 1+\lambda_{m}
\end{array}\right)\left(\begin{array}{cccc}
b_{21} & 0 & \ldots & 0 \\
0 & b_{22} & \ldots & 0 \\
& & \ldots & \\
0 & 0 & \ldots & b_{2 m}
\end{array}\right)
\end{aligned}
$$

At last, we have for $\mu=\operatorname{diag}\left(b_{21}, b_{22}, \ldots, b_{2 m}\right)^{-1} b=\left(\frac{b_{11}}{b_{21}}, \frac{b_{12}}{b_{22}}, . ., \frac{b_{1 m}}{b_{2 m}}\right)$

$$
\left(A_{(m)}^{-1} b, b\right)=\left(D^{-1}\left(\lambda_{1}, \lambda_{2}, \ldots, \lambda_{m}\right) \mu, \mu\right), \quad \lambda_{k}=\left(1+\frac{b_{1 k}}{b_{2 k}}\right)^{2}-1, \quad \mu_{k}=\frac{b_{1 k}}{b_{2 k}}
$$


Using Lemma 4.23 for the operator $A_{2 m+1}$, and the vector $b \in \mathbb{R}^{2 m+1}$ we obtain

$$
\left(A_{2 m+1}^{-1} b, b\right)=\frac{\sum_{k=-m}^{m} \frac{\left(\frac{b_{1 k}}{b_{2 k}}\right)^{2}}{\left(\frac{b_{1 k}}{b_{2 k}}+1\right)^{2}-1}+\sum_{-m \leq k<n \leq m} \frac{\left(\frac{b_{1 k}}{b_{2 k}}-\frac{b_{1 n}}{b_{2 n}}\right)^{2}}{\left[\left(\frac{b_{1 k}}{b_{2 k}}+1\right)^{2}-1\right]\left[\left(\frac{b_{1 n}}{b_{2 n}}+1\right)^{2}-1\right]}}{\sum_{k=-m}^{m} \frac{1}{\left(\frac{b_{1 k}}{b_{2 k}}+1\right)^{2}-1}+1}=
$$

$\Delta\left(f_{m}^{1}, g_{m}^{1}\right)$ where

$$
f_{m}^{1}=\left(\frac{b_{1 k}}{\sqrt{b_{1 k}^{2}+2 b_{1 k} b_{2 k}}}\right)_{k=-m}^{m}, \quad g_{m}^{1}=\left(\frac{b_{2 k}}{\sqrt{b_{1 k}^{2}+2 b_{1 k} b_{2 k}}}\right)_{k=-m}^{m}
$$

The proof of Lemma 4.3 is similar. We get $\left(A^{-1} b, b\right)=\Delta\left(f_{m}^{2}, g_{m}^{2}\right)$ where

$$
f_{m}^{2}=\left(\frac{b_{2 k}}{\sqrt{b_{2 k}^{2}+2 b_{1 k} b_{2 k}}}\right)_{k=-m}^{m}, \quad g_{m}^{2}=\left(\frac{b_{1 k}}{\sqrt{b_{2 k}^{2}+2 b_{1 k} b_{2 k}}}\right)_{k=-m}^{m} .
$$

Acknowledgements. The author expresses his deep gratitude to the Max Planck Institute for Mathematics for the financial grant and the hospitality in 2016-2017.

\section{References}

[1] S. Albeverio and A. Kosyak, Quasiregular representations of the infinitedimensional Borel group, J. Funct. Anal. 218/2 (2005) 445-474.

[2] S. Albeverio and A. Kosyak, Quasiregular representations of the infinitedimensional nilpotent group, J. Funct. Anal. 236 (2006) 634-681.

[3] E.F. Beckenbach and R. Bellmann, Inequalities, Springer, Berlin, Göttingen, Heidelberg, 1961.

[4] Yu. M. Berezanskii, Selfadjoint operators in spaces of functions of infinitely many variables. Translated from the Russian by H. H. McFaden. Translations of Mathematical Monographs, 63. AMS, Providence, RI, 1986. 
[5] J. Dixmier, Les $C^{*}$ algèbres et leur représentations, Gautier-Villars, Paris, 1969.

[6] J. Dixmier, Les algèbres d'opérateurs dans l'espace hilbertien, 2nd Edition, Gauthier-Villars, Paris, 1969.

[7] R.F. Gantmacher, Matrizenrechnung. Teil 1. Veb Deutscher Verlag der Wissenschaften, Berlin, 1958.

[8] S. Helgason, Groups and geometric analysis. Integral geometry, invariant differential operators and spherical functions. Mathematical Surveys and Monographs, v 83, Academic Press, Orlando, 1984.

[9] R.A. Horn, C.R. Jonson, Matrix analysis, Cambridge University Press, Cambridge, 1989.

[10] R.A. Horn, C.R. Jonson, Topics in Matrix analysis, Cambridge University Press, Cambridge, 1991.

[11] S. Kakutani, On equivalence of infinite product measures, Ann. Math. 4 (1948) 214-224.

[12] A.W. Knapp, Representation theory of semisimple groups, An Overview Based on Examples, Princeton University Press, Princeton, New Jersey, 1986 ,

[13] B.O. Koopman, Hamiltonian systems and transformation in Hilbert space, Proc. Natl. Acad. Sci. U. S. A. 17 (1931), no. 5, 233-238.

[14] A.V. Kosyak, Irreducibility criterion for regular Gaussian representations of groups of finite upper triangular matrices, Funct. Anal. Appl. 24 (1990) no. 3, 243-245.

[15] A.V. Kosyak, Criteria for irreducibility and equivalence of regular Gaussian representations of group of finite upper triangular matrices of infinite order, Selecta Math. Soviet. 11 (1992) 241-291.

[16] A.V. Kosyak, Irreducible regular Gaussian representations of the group of the interval and the circle diffeomorphisms, J. Funct. Anal. 125 (1994) $493-547$. 
[17] A.V. Kosyak, The generalized Ismagilov conjecture for the group $B_{0}^{\mathbb{N}}$. I, Methods Funct. Anal. Topology. 8, No 2 (2002) 33-49.

[18] A.V. Kosyak, The generalized Ismagilov conjecture for the group $B_{0}^{\mathbb{N}}$. II, Methods Funct. Anal. Topology. 8, No 3 (2002) 27-45.

[19] A.V. Kosyak, Irreducibility criterion for quasiregular representations of the group of finite upper-trianguler matrices. in Funct. Anal. Appl. 37 (2003) no. 1, 65-68.

[20] A.V. Kosyak, Quasi-invariant measures and irreducible representations of the inductive limit of the special linear groups, Funct. Anal. Appl. 38 (2004) no.1, 67-68.

[21] A.V. Kosyak, Regular, quasiregular and induced representations of infinite-dimensional groups, Doctoral Dissertation, Kiev Institute of mathematics, June, 2010 (in Ukrainian).

[22] A.V. Kosyak, Induced representations of infinite-dimensional groups, I, J. Funct. Anal, 266 (2014) 3395-3434.

[23] H.H. Kuo, Gaussian measures in Banach spaces, in: Lecture Notes Mathematics, Vol. 463, Springer, Berlin, 1975.

[24] I. Penkov and K. Styrkas, Tensor representations of classical locally finite Lie algebras, in: Developments and Trends in Infinite-Dimensional Lie Theory, Progress in math, 288, K.-H. Neeb and Pianzola (eds.) Springer Basel, 2011.

[25] I. Schur, Über eine Klasse von Matrizen, die sich einer gegebenen Matrix zuordnen lassen. Dissertation. Berlin. 76 S (1901) JMF 32.0165.04

[26] I. Schur, Über die rationalen Darstellungen der allgemeinen linearen Gruppe. Sitzungsberichte Akad. Berlin 1927, 58-75 (1927) JMF 53.0108 .05

[27] Tsilevich, N.V. and Vershik, A.M. Infinite-dimensional Schur-Weyl duality and the Coxeter-Laplace operator, Commun. Math. Phys., 327, Issue 3, (2014) 873-885.

[28] G.E. Shilov and Fan Dik Tun', Integral, Measure, and Derivative on Linear Spaces (Russian), Nauka, Moscow, 1967. 
[29] A.V. Skorokhod. Integration in Hilbert space, Springer, Berlin, 1974.

[30] A. Weil, L'intégration dans les groupes topologique et ses application $2^{e}$ ed., Hermann, Paris, 1953.

[31] H. Weyl, The Classical Groups. Their Invariants and Representations. Princeton University Press, Princeton, N.J., 1939. xii+302 pp.

[32] Q. Yuan, Four flavors of Schur-Weyl duality, https://qchu.wordpress. com/2012/11/13/four-flavors-of-schur-weyl-duality/ 